\title{
Taxonomic revision of the genus Crassicrus Reichling \& West, 1996 (Araneae: Theraphosidae: Theraphosinae), with the description of additional keels on the embolus
}

\author{
Daniela T. Candia-Ramírez and Oscar F. Francke: Colección Nacional de Arácnidos, Departamento de Zoología, \\ Instituto de Biología, Universidad Nacional Autónoma de México, Coyoacán, Ciudad de Mexico, Mexico. E-mail: \\ brachypelma_boehmei04@hotmail.com
}

\begin{abstract}
Since its original description, the theraphosid spider genus Crassicrus Reichling \& West, 1996 has not been revised and no new species have been described. While reviewing material deposited in the Mexican National Collection of Arachnids (National Autonomous University of Mexico, Mexico City) and the American Museum of Natural History (New York, USA), we encountered specimens corresponding to four new species of Crassicrus from Mexico. In this revision, we include a redescription of the genus and its type species, C. lamanai Reichling \& West, 1996, and describe four new species: $C$. bidxigui, $C$. tochtli, C. cocona, and C. yumkimil. Species habitat data are provided, as well as identification keys for males and females. In addition, new keels on the male embolus were identified and are described. In the Theraphosinae, the presence of one retrolateral keel has been reported, but in Crassicrus, there are two or three retrolateral keels, and a new taxonomical nomenclature for these keels is proposed. The genus Crassicrus is recorded from Mexico for the first time, increasing the number of known theraphine genera in the country to 16 .
\end{abstract}

Keywords: Taxonomy, embolus keels, spermatic pore, morphology, new species

http://zoobank.org/?lsid=urn:lsid:zoobank.org:pub:76D547C2-07FA-4998-A673-582B7F4DF028

The morphology of the genitalia in Theraphosidae, and in Mygalomorphae more generally, has not been studied in detail, although genitalia provide important features used in the classification and identification of species (Bücherl 1957; Schiapelli \& Gerschman 1962; Gerschman \& Schiapelli 1970; Goloboff 1993; Ortiz \& Francke 2014). The most diverse subfamily of the Theraphosidae is the Theraphosinae Thorell, endemic to the Americas. The structure of the male palpal bulb has been analyzed for some genera of this subfamily and some primary homology hypotheses for the keels on the bulb have been established (Pérez-Miles et al. 1996; Bertani 2000). According to Bertani (2000), in theraphosine palpal bulbs there are four major groups of keels: (1) the prolateral keels (superior and inferior); (2) the apical keel; (3) the subapical keel; and (4) the retrolateral keel. The taxonomy of Theraphosinae is problematic and most genera lack revisions or are diagnosed based on relatively few characters (Raven, 1985, 1990; Smith 1995; Pérez-Miles et al. 1996; Prentice 1997; Fukushima et al. 2008; Yamamoto et al. 2012). Some factors that contribute to this problematic taxonomy are the morphological uniformity among members of this group, the small number of traditional taxonomists working on Theraphosinae, and the subfamily's broad geographic distribution (Schiapelli \& Gerschman 1979; Valerio 1980; Raven 1985, 1990; Goloboff 1993; Pérez-Miles et al. 1996; Bertani 2000, 2001). The revision of genitalic features as well as of other structures is important in order to increase our knowledge and understanding of this group, and to propose homologies among characters for the numerous theraphosine genera (Bücherl 1957; Schiapelli \& Gerschman 1962; Bertani 2000; Ortiz 2008). This is especially so as the number of revisions and taxonomic works involving theraphosids has increased over the last decade. However, in North America there have been relatively few studies in comparison to the known diversity of the region. Mexico is the second most diverse country in terms of known tarantula species worldwide and this number has been increasing annually, with new descriptions displaying the growing importance and diversity of North American theraphosid species (Locht 2007; World Spider Catalog 2016). The only North American genera that have been revised, or partially revised to date are Aphonopelma Pocock, 1901, Hemirrhagus Simon, 1903, Bonnetina Vol, 2000, and Brachypelma Simon, 1891 (see Hamilton et al. 2011, 2016; Mendoza 2012; Mendoza 2014; Ortiz \& Francke 2014; Ortiz \& Mendoza, pers. comm.).

With respect to Belize and southeastern Mexico, the theraphosid fauna has not been studied in detail. According to Reichling (2003) and the World Spider Catalog (2016), there are only nine species reported from Belize and eight from southeastern Mexico. One of the genera reported for Belize is Crassicrus Reichling \& West, 1996, which is a monotypic genus with C. lamanai Reichling \& West, 1996. With the revision of biological material deposited in the Mexican National Collection of Arachnids (CNAN) (National Autonomous University of Mexico, Mexico City; UNAM) and the American Museum of Natural History (New York, USA; AMNH), we found specimens corresponding to four undescribed species of Mexican Crassicrus.

In this contribution, we present the first revision of the genus Crassicrus, including a redescription of the type species C. lamanai, a redescription and comparative diagnosis of the genus, and descriptions of four new species. This is the first record for the genus in Mexico. In addition, we found embolic keels on the males of Crassicrus that have not been reported before, and we describe these and report other features of the male genitalia that were not widely reported previously. The purpose of this work, in addition to the generic revision, is to contribute to the knowledge of Theraphosidae and provide new characters for future studies on the systematics of the family. 


\section{METHODS}

The general description format follows Bertani et al. (2011). A Nikon SMZ 625 stereomicroscope was used for the observation of specimens and structures; urticating setae were examined with a Nikon Eclipse E100 compound microscope. Digital images were taken with a Nikon Coolpix S10 VR digital camera, with an adapter for the stereomicroscope. Male palpal bulbs and small sections of exuviae were dissected, critical-point dried, gold coated, and examined at low vacuum in Hitachi S-2460N and SU1510 scanning electron microscopes (SEM) at UNAM. Measurements are given in millimeters $(\mathrm{mm})$, except SEM measurements which are in micrometers $(\mu \mathrm{m})$; all measurements were taken along the central axis of the structures, in smaller structures with an ocular micrometer attached to the microscope, and in larger ones with a digital caliper with an error of $0.1 \mathrm{~mm}$. Size ranges are given in millimeters $(\mathrm{mm})$. Total body length was measured excluding the chelicerae and spinnerets. The leg span was measured with the legs fully extended, and was measured from the apex of tarsus I to the apex of tarsus IV of the side where the legs would be least damaged. Leg and pedipalp measurements were taken from the left appendages, except in the case of absence or damage. The width of all segments was measured laterally at the midpoint of each segment; specific leg segments having a considerably greater width in comparison with the same segment on different appendages were considered and described as thickened. See Appendix 1 for comparative material examined as part of this study.

Spination and setae. - Spination descriptions follow Bertani (2001). The classification of urticating setae follows Bertani \& Guadanucci (2013). The description of the lateral scopulae follows Mendoza (2014), adding the presence of thin plumose setae. The metatarsal scopulae are described in percentages, considering the proportion of the segment length that is covered with scopulae. For leg spination, only the surfaces with spines are mentioned.

Pedipalp. - The terminology of the male palpal bulb keels follows Bertani (2000) with the following modifications: in Crassicrus there are two or three keels on the retrolateral face of the embolus; for comparisons of these keels between the different species, every keel was named; for the keels of the spermatic pore, we follow Ortiz \& Francke (2014).

Mapping.-The distribution map was created using gvSIG version 2.1.0, using geographical and political division layers downloaded from Natural Earth (2015). The geographical coordinates were obtained in the field with an Etrex GPS. For localities that were not directly sampled or those that only had distance data, Google Earth version 7.1.2.2041 was used for the geo-referencing of localities and to estimate distances based on label landmarks.

Abbreviations.-Abbreviations follow Bertani (2000) and Ortiz \& Francke (2014) for male palpal bulb keels; Coyle (1995) for spermathecae; Raven (1985) for somatic characters; Bertani \& Guadanucci (2013: fig. 2) for the abdominal region; and Mendoza (2014) for tibial apophyses, as follows: a, apical; A, apical keel; ALE, anterior lateral eyes; AME, anterior median eyes; d, dorsal; MA, median anterior region of abdomen; MM, median region of abdomen; $p$, prolateral; Pap, prolateral branch of leg I tibial apophysis; PI, prolateral inferior keel; PLE, posterior lateral eyes; PME, posterior median eyes; PLS, posterior lateral spinnerets; PMS, posterior median spinnerets; PS, prolateral superior keel; r, retrolateral; Rap, retrolateral branch of leg I tibial apophysis; RI, retrolateral inferior keel; RM, retrolateral median keel; RS, retrolateral superior keel; SA, subapical keel; SB, spermathecae bulb; SP, spermatic pore keel; SS, spermathecal stalk; v, ventral.

\section{SYSTEMATICS}

Family Theraphosidae Thorell, 1870

Subfamily Theraphosinae Thorell, 1870

Genus Crassicrus Reichling \& West, 1996

Crassicrus Reichling \& West, 1996: 254.

Type species.-Crassicrus lamanai Reichling \& West, 1996, by original designation.

Diagnosis (emended).-Males and females of Crassicrus can be distinguished from all other genera of Theraphosinae except Aphonopelma, Citharacanthus Pocock, 1901, Cyrtopholis Simon, 1892, Lasiodora C. L. Koch, 1850, Megaphobema Pocock, 1901, Sphaerobothria Karsch, 1879, Stichoplastoris Rudloff, 1997 and Vitalius Lucas, Silva \& Bertani, 1993 by the presence of only urticating setae type I on the dorsal surface of the abdomen (Cooke et al., 1972) (Fig. 1). Males and females can be distinguished from these genera by presenting cuneiform thorn-like setae on the prolateral face of coxae IIV, which are thicker near the ventral region (Figs. 1E, 7F, 8G, $11 \mathrm{G}, 13 \mathrm{E}, 14 \mathrm{H}, 16 \mathrm{~F}, 17 \mathrm{G}$ ), and by having the labio-sternal mounds semicircular and well separated (Figs. 5B, 7B, 8B, 10B, 11B, 13B, 14B, 16B, 17B). Males are further distinguished by having the following combination of characters: palpal bulb with the proximal region of the tegulum rounded (Figs. 6B, 9B, 12B, 15B, 18B); tibia with two apophyses which do not originate from a common base (Figs. $5 \mathrm{G} \& \mathrm{H}, 8 \mathrm{H}$, $11 \mathrm{H}, 14 \mathrm{G}, 17 \mathrm{H}$ ); and palpal bulb with proventral face of subapical region of embolus (between the PI and SA keels) convex (Figs. 6A, 9A, 12A, 15A, 18A). In other Theraphosinae species, e.g., Aphonopelma anitahoffmannae Locht, Medina, Rojo \& Vázquez, 2005, this subapical region is flat, and in species like Eupalaestrus weijenberghi (Thorell, 1894) and Vitalius sorocabae (Mello-Leitão, 1923), it is slightly concave. Females are distinguished for the presence of spiniform setae on the ventral and proventral surfaces of femora II-IV (Figs. 7G, 10G, 13I, 16I), and two spermathecae partially fused by a heavily sclerotized median region, with the $\mathrm{SB}$ as wide as or wider than long (Figs. $7 \mathrm{I} \& \mathrm{~J}, 10 \mathrm{H}, 13 \mathrm{~J}, 16 \mathrm{H}$ ).

Description.- Total length: males $28-37 \mathrm{~mm}$; females $35-49$ $\mathrm{mm}$.

Prosoma: Female carapace dark brown; male carapace dark brown to black with coppery and violet iridescent setae. Cephalic region slightly darker than pars thoracica. Carapace widest at level of coxae II-III. Caput slightly elevated. Fovea variable: straight (Figs. 10A, 13A), slightly recurved (Figs. 5A, $11 \mathrm{~A}, 14 \mathrm{~A}, 17 \mathrm{~A}$ ), or slightly procurved (Fig. 16A). Anterior eye row straight or procurved; posterior eye row recurved. Clypeus very narrow. Cheliceral prolateral furrow with 11 to 16 teeth. Labium wider than long; anterior region with 24 to 122 cuspules. Labio-sternal mounds semicircular, separated from each other by less than half of their width. Maxillae 

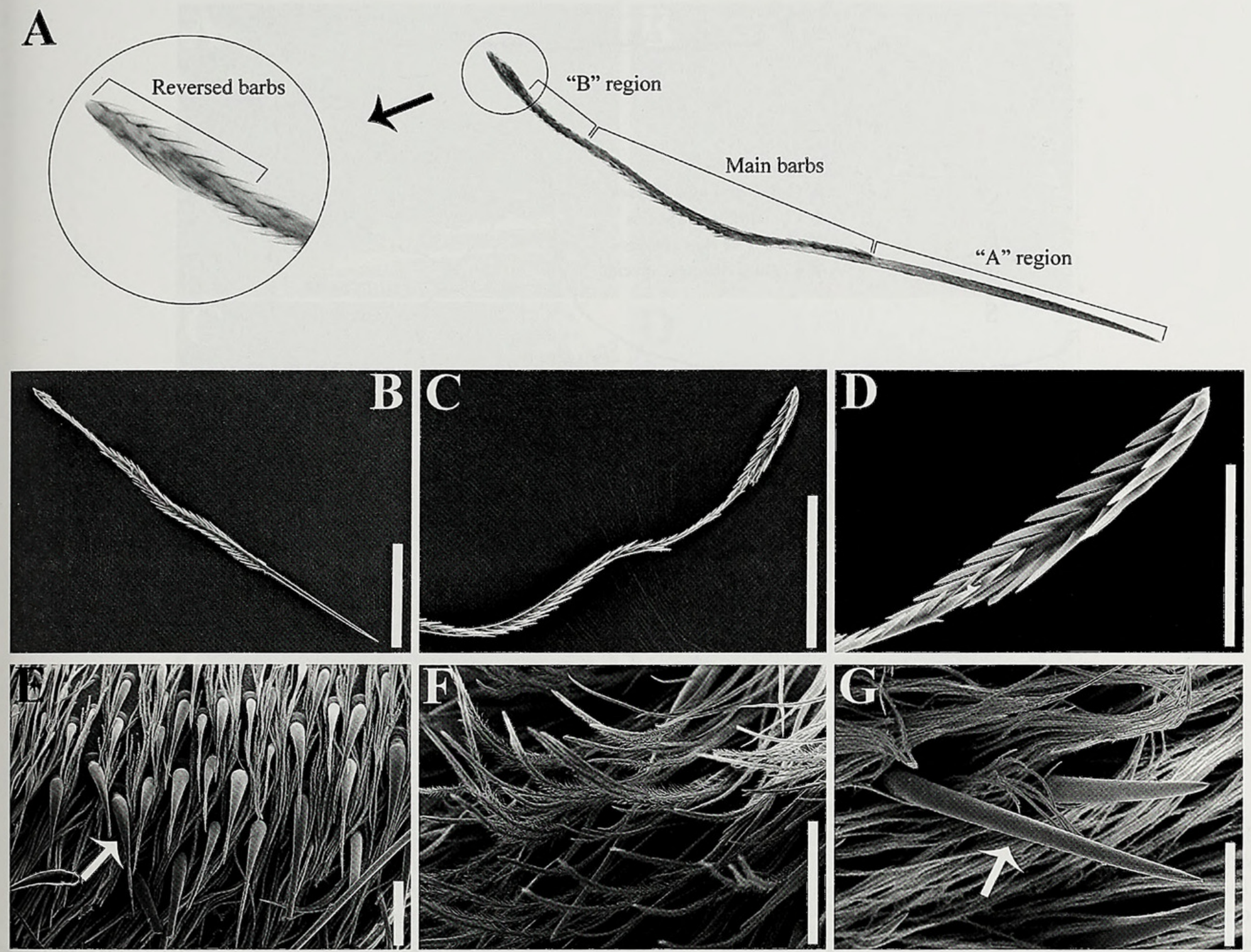

Figure 1.- Important morphological features found in Crassicrus. A. Modified urticating setae type I of median region of abdomen of $C$. cocona sp. nov. B-D. Urticating setae type I of median region of abdomen of C. yumkimil sp. nov.: B. Urticating setae type I with region "A" longer than "B". C. Detail of main barbs, "B" region, and reversed barbs. D. Detail of reversed barbs. E. Conical spiniform setae (arrow) on prolateral face of coxa I of male $C$. cocona sp. nov. F. Thin plumose setae on prolateral face of femur I of male $C$. cocona sp. nov. G, Elongated spiniform setae (arrow) on prolatero-ventral face of femur III of female of Crassicrus sp. Scale bars: $40 \mu \mathrm{m}(\mathrm{D}), 100 \mu \mathrm{m}(\mathrm{B}-\mathrm{C}), 200 \mu \mathrm{m}$ (E-G).

longer than wide, with 124 to 290 cuspules on baso-prolateral region. Sternum longer than wide; posterior margin not extending between coxae IV. Sternum convex (Figs. 8B, 11B, 14B) or flat (Figs. 5B, 17B); sigilla present (Figs. 5B, 17B) or absent (Figs. 10B, 13B); if present, they are close to basal retrolateral region of coxae I-III, and the third pair is the largest (Figs. 5B, 14B, 17B).

Legs: Leg formula: IV, I, II, III. In females and juveniles, the patellae, tibiae, metatarsi, and tarsi of the pedipalps and legs III are light brown, whereas on legs III and IV these segments are dark brown to black (Fig. 4B, C, E, G); the femora of the pedipalps and legs are black. In males, the legs are uniformly black, and can have violet iridescent setae on dorsal regions of the coxae, trochanters and femora (Fig. 4A, D, F). Retrolateral surface of palpal trochanter, prolateral surfaces of trochanter I and femur I, and prolateral surfaces of trochanter II and femur II are covered with long, thin, plumose setae (Fig. 1F).
Prolateral surfaces of coxae I-IV are covered with short, cuneiform thorn-like setae that are thicker near the ventral region (Figs. 1E, 7F, 8G, 10F, 11G, 13E, 14H, 16F, 17G). Retrolateral surfaces of maxillae and coxae I-III sparsely covered by very short spiniform setae (Figs. 8F, 13G, 17I). Females present long spiniform setae on the proventral surfaces of femora II-IV (Figs. 1G, 7G, 10G, 13I, 16I). Tibiae IV can be slightly to very incrassate (Fig. $7 \mathrm{H}$ ) or not at all. Male metatarsus I straight; when flexed, it touches the lateral external face of the retrolateral branch of leg I tibial apophysis. Tarsal scopulae undivided, in some species the tarsal scopulae of leg IV can be divided by a longitudinal row of longer setae.

Tibial apophyses: Male tibia I bears two branches, with separated bases: the prolateral (Pap) is digitiform, and in some species thickened (Fig. 11H); the retrolateral (Rap) is longer than Pap and slightly curved distally towards the Pap (Figs. $5 \mathrm{G}, 8 \mathrm{H}, 11 \mathrm{H}, 14 \mathrm{G}, 17 \mathrm{H})$. 


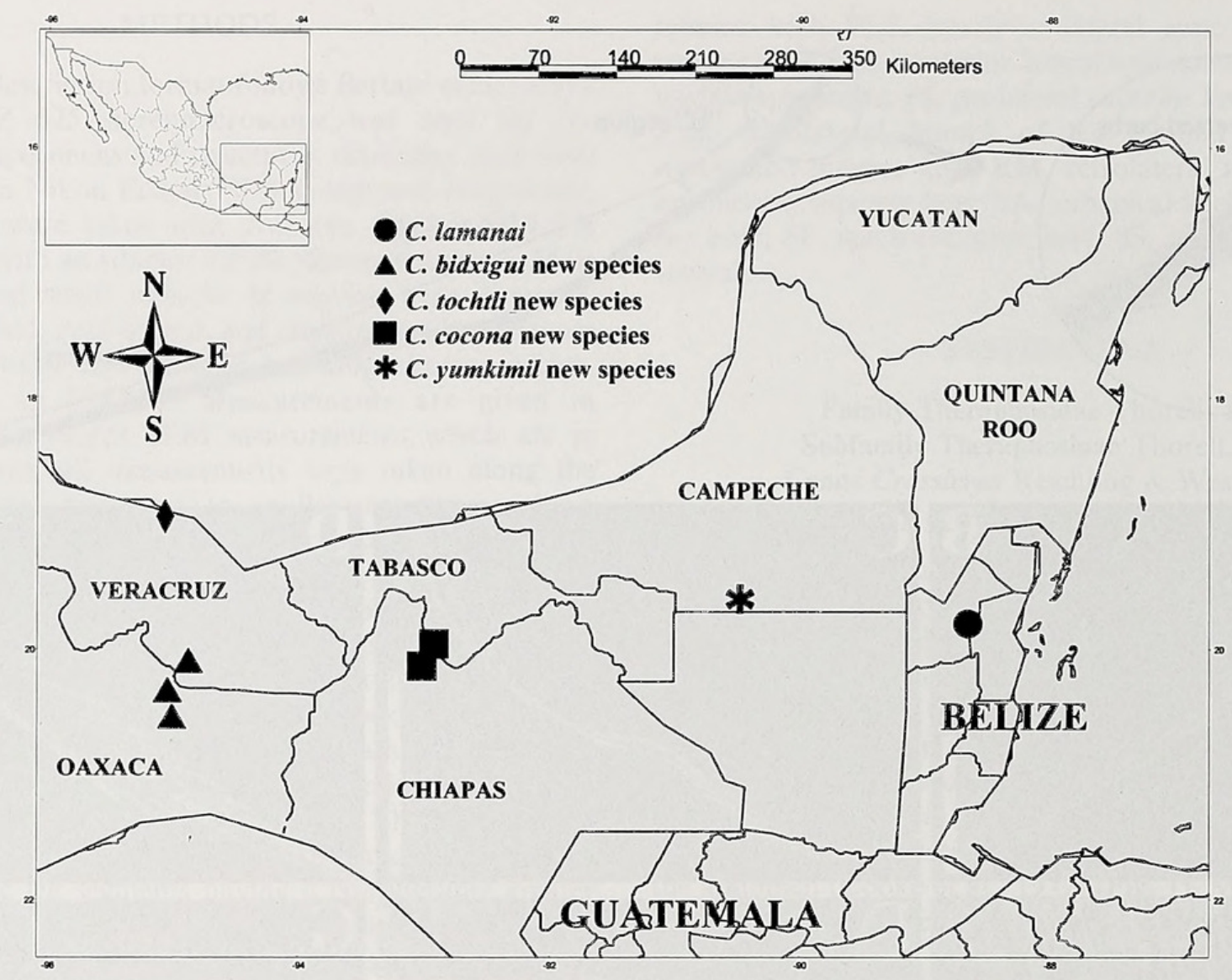

Figure 2.-Distribution map of the species of the genus Crassicrus.

Opisthosoma: Abdomen with dorsal surface covered with short dark setae, giving it a velvety appearance, interspersed with longer yellow to orange setae (Figs. 7C, 10C, 13C, 16C). Under the short setae, there is coppery brown pubescence corresponding to the urticating setae patch and extending posteriorly to two-thirds the length of the abdomen. Ventral region covered by numerous short black setae.

Urticating setae: Type I (Fig. 1), with region "A" larger than "B" (Fig. 1B). In most species, the males have considerably longer urticating setae on area $\mathrm{MM}$ in comparison to those of area MA (Figs. 1C, 1D); with region "A" very long (Fig. 1B). In some species, the urticating setae type I are modified and the region with reversed barbs is very short (Fig. 1A).

Male pedipalpal bulb: Embolus short, subapical dorsal region concave. In some species, the median ventral region is flat and, in others, it presents a shallow depression (Figs. 6A, $18 \mathrm{~A})$. Some species present striations on the prolateral upper face (Figs. 9A, 15A, 18A) or on the ventral region near the embolus (Fig. 12F). The embolus presents eight or nine keels, including: an apical keel (A), which is very reduced (Figs. 6E, 9F, 12E, 15E, 18E); a subapical keel (SA), which is serrated, and distally curves towards the retrolateral face of the embolus (Figs. 6B, 9D, 12D, 15B, 18D); and two prolateral keels (PI and PS) (Figs. 6A, 9A, 12A, 15A, 18A). Some species have two keels on the retrolateral face of the embolus: a retrolateral median (RM) keel, and a retrolateral inferior (RI) keel (Figs. $9 \mathrm{~B}, 12 \mathrm{~B}, 15 \mathrm{~B}, 18 \mathrm{D})$. The retrolateral median keel is located on the medial portion of the retrolateral face of the embolus; in some species, this keel is distally connected to the prolateral keels, forming the embolus tip. The retrolateral inferior keel is located on the inferior half of the retrolateral face of the embolus, between the retrolateral median and subapical keels, and it is weakly sclerotized distally. Other species, in addition to having the RM and RI keels, have a third retrolateral keel (Fig. 6B). The retrolateral superior (RS) keel shapes the dorsal edge of the embolus and is generally heavily sclerotized on its median portion; in some species, this keel is distally connected to the prolateral superior keel, and together they form the tip of embolus. Finally, two curved keels surround the spermatic pore (Figs. 6E, 9F, 12E, 15E, 18E).

Spermathecae: Female genitalia consisting of two receptacles partially fused by a heavily sclerotized median region. In some species, the median region is wider and slightly curved on its upper portion (Figs. 10H, 13J), whereas in others the median region has the same width throughout (Figs. 7J, 16H). The SB are as wide as or wider than long.

Composition.-Crassicrus lamanai, C. bidxigui sp. nov., C. tochtli sp. nov., C. cocona sp. nov., and $C$. yumkimil sp. nov.

Distribution.-Crassicrus is known from only five localities situated between latitudes $19^{\circ} \mathrm{N}$ and $21^{\circ} \mathrm{N}$, from western Oaxaca (Isthmus of Tehuantepec) and southern Veracruz in Mexico, east to northern Belize (Fig. 2).

Natural history.--Spiders of the genus Crassicrus are known from locations with a tropical climate and elevations below $250 \mathrm{~m}$. Their typical habitat is open areas, where native vegetation (mainly tropical rainforest and deciduous forest) has been replaced by roads, agricultural fields and pastures (Fig. 3A, C, D, F). Despite intensive collection efforts, specimens of Crassicrus were not found in highly preserved areas as Reichling \& West (1996) mentioned; this could be due 

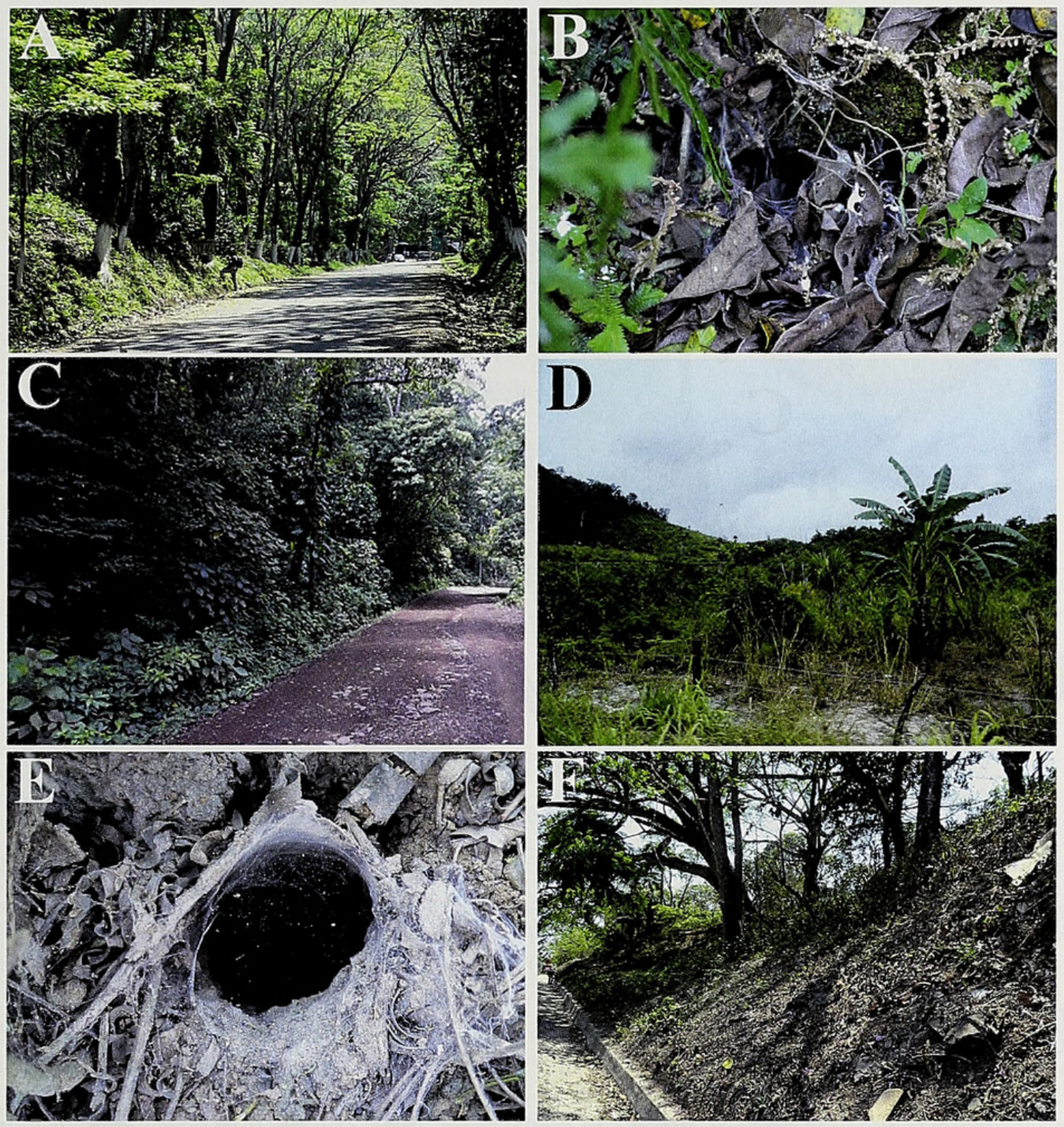

Figure 3.-Crassicrus species, habitats and burrows: A. Rainforest on road to Grutas de Coconá, Tabasco, Mexico, type locality of C. cocona sp. nov. B. Burrow of $C$. cocona sp. nov. C. Rainforest close to Los Tuxtlas Biological Station Veracruz, Mexico, type locality of $C$. tochtli sp. nov. D. Deciduous forest $1 \mathrm{~km} \mathrm{~W}$. of El Pañuelo, Campeche, Mexico, type locality of $C$. yumkimil sp. nov. E. Burrow of $C$. bidxigui sp. nov. F. Deciduous forest at Piedra Blanca, San Juan Guichicovi, Oaxaca, Mexico, locality of $C$. bidxigui sp. nov.

to their preference for exposed sunny terrains over shadowy areas with dense vegetation. Most of the specimens of Crassicrus were found in burrows located along the borders of crop fields; the burrows are about 30 to $40 \mathrm{~cm}$ deep, with a circular entrance and sparsely covered with a layer of silk (Fig. $3 \mathrm{~B}, \mathrm{E})$. It is worth noting that only adult females and juvenile males were obtained from burrows, and in some cases, juveniles were obtained by searching under rocks. Juvenile males were raised in captivity until they became sexually mature, usually in the rainy season from July to September.
Considering the material deposited in collections, the reproductive season seems to occur from August to January, because adult males were collected during those months wandering near roadsides. All of the species of Crassicrus from known localities are sympatric with species of Brachypelma whose individuals are also burrowers, and clearly more abundant (pers. obs.) than those of Crassicrus. In Los Tuxtlas (Veracruz, Mexico) Crassicrus was also found to be sympatric with a non-digging tarantula species tentatively assigned to the contentious genus Citharacanthus.

\section{KEYS TO THE KNOWN SPECIES OF CRASSICRUS}

\section{MALES}

1. Sternum flat (Figs. 5B, 17B); ventral region of bulb with a shallow depression (Figs. 6A, 18A) . . . . . . . . . . . . 2

Sternum convex (Figs. 8B, 11B, 14B); ventral region of bulb without a shallow depression (Figs. 9A, 12A, 15A) ...... 3 


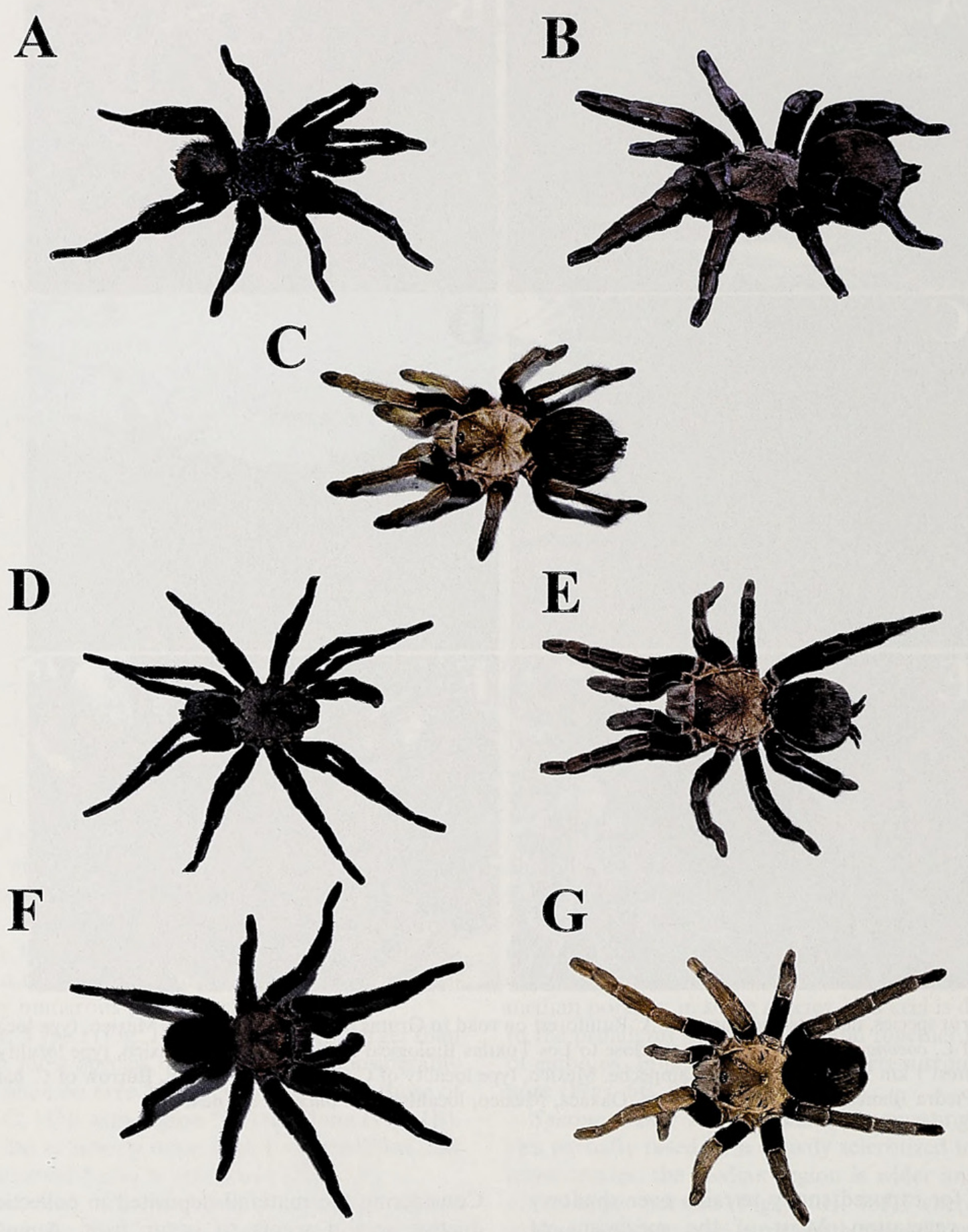

Figure 4.-Crassicrus species, habitus images. A-B. Crassicrus lamanai: A. Male. B. Female from $0.5 \mathrm{~km}$ W. of New River Lagoon, Indian Church Village, near Lamanai Forest Reserve, Orange Walk District, Belize. C. Crassicrus bidxigui sp. nov. female from Tolosa Donají, Matías Romero Avendaño, Oaxaca, Mexico. D-E. Crassicrus tochtli sp. nov.: D. Male (CNAN-T0898). E. Female from Biological Station "Los Tuxtlas", San Andrés Tuxtla, Veracruz, Mexico. F-G. Crassicrus cocona sp. nov.: F. Male (CNAN-T0894). G. Female (CNAN-T0895) from road to Grutas de Coconá, Teapa, Tabasco, Mexico. Photos A \& B by Rick C. West.

2. Palpal bulb's prolateral face with striations (Fig. 18A); retrolateral face with two parallel keels (RM and RI) (Fig. 18D)

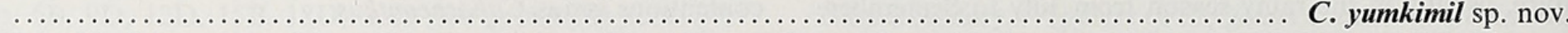
Palpal bulb's prolateral face without striations (Fig. 6A); retrolateral face with three parallel keels (RS, RM and RI) (Fig. 6B)

C. lamanai

3. Urticating setae type I modified, with the region of the reversed barbs very reduced (Fig. 14J); RI keel of palpal bulb bearing denticles on the proximal region (Fig. 15F) . C. cocona sp. nov. Urticating setae type I not modified, with the region of the reversed barbs not reduced (Fig. 1D); RI keel of palpal bulb without denticles on proximal region 

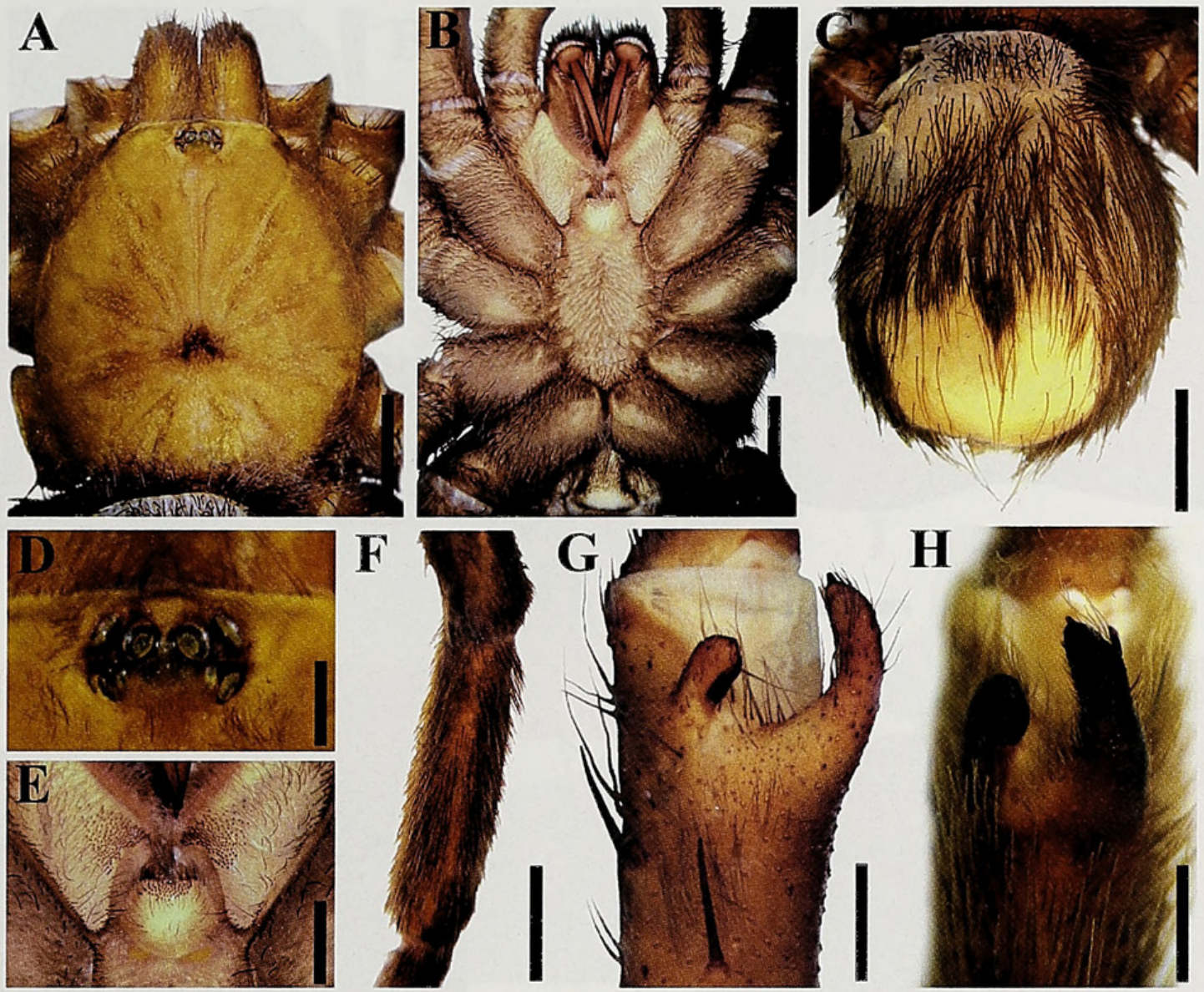

Figure 5.-Crassicrus lamanai male morphology. A-G. Holotype: A. Carapace. B. Prosoma, ventral view. C. Abdomen, dorsal view. D. Ocular tubercle. E. Labium, maxillae, and labio-sternal mounds. F. Tibia IV, dorsal view. G. Tibial apophyses, ventro-prolateral view. H. Paratype, tibial apophyses, ventral view. Scale bars: $1 \mathrm{~mm}(\mathrm{D}), 2 \mathrm{~mm}(\mathrm{G}-\mathrm{H}), 2.5 \mathrm{~mm}(\mathrm{E}), 5 \mathrm{~mm}(\mathrm{~A}-\mathrm{C}, \mathrm{F})$.

4. Coxae with basal spiniform setae on prolateral faces, these setae longer and wider on coxae III and IV; palpal bulb with striations on prolateral face (Fig. 9A), without striations on ventral surface; leg I tibial apophyses with Pap not thickened

C. bidxigui sp. nov.

Spiniform setae fully covering prolateral faces of coxae, these setae slightly shorter and thinner on coxae III and IV; palpal bulb without striations on prolateral face, with striations on ventral surface near the embolus (Fig. 12F); leg I tibial apophyses with Pap thickened $($ Fig. $11 \mathrm{H}) \ldots \ldots \ldots \ldots \ldots \ldots \ldots \ldots \ldots \ldots \ldots \ldots \ldots \ldots \ldots \ldots \ldots \ldots \ldots \ldots$, tochtli sp. nov

\section{FEMALES}

NB. Females of $C$. yumkimil sp. nov. are unknown

1. Tibia IV thickened with respect to other leg segments (Fig. 7H); sternum flat (Fig. 7B)

C. lamanai Tibia IV not thickened with respect to other leg segments; sternum convex (Figs. 10B, 13B, 16B)....

2. Spermathecae without broad upper edge on median region (Fig. 16H) $\ldots \ldots \ldots \ldots \ldots \ldots \ldots \ldots \ldots \ldots$. cocona sp. nov

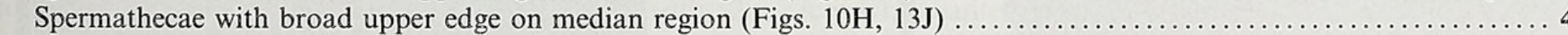

4. Coxae with basal spiniform setae on prolateral faces, these setae longer and wider on coxae III and IV; femur IV longer than metatarsus IV ........................................................ bidxigui sp. nov. Spiniform setae fully covering prolateral faces of coxae, these setae slightly shorter and thinner on coxae III and IV; femur IV shorter than metatarsus IV

C. tochtli sp. nov.

\section{Crassicrus lamanai Reichling \& West, 1996} (Figs. 5-7)

Crassicrus lamanai Reichling \& West, 1996: 254, figs. 1-9; Schmidt, 1997: 19, figs. 187-189; Vol, 1999: 11, fig. D; Schmidt, 2003: 136, figs. 198-200; Schmidt, 2007a: 8, figs. 1, 2; Schmidt, 2007b: 100, figs. 1, 2.
Type material.-Holotype male. BELIZE: Orange Walk District: $0.5 \mathrm{~km}$ W. of New River Lagoon, Indian Church Village, near Lamanai Forest Reserve, 6 January 1995, S.B. Reichling (AMNH).

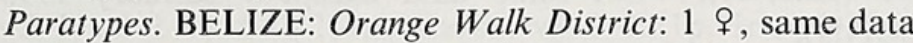
as holotype (AMNH); 1 o, same data except 7 January 1995 (AMNH; not examined); 2 o, same data except 3 September 

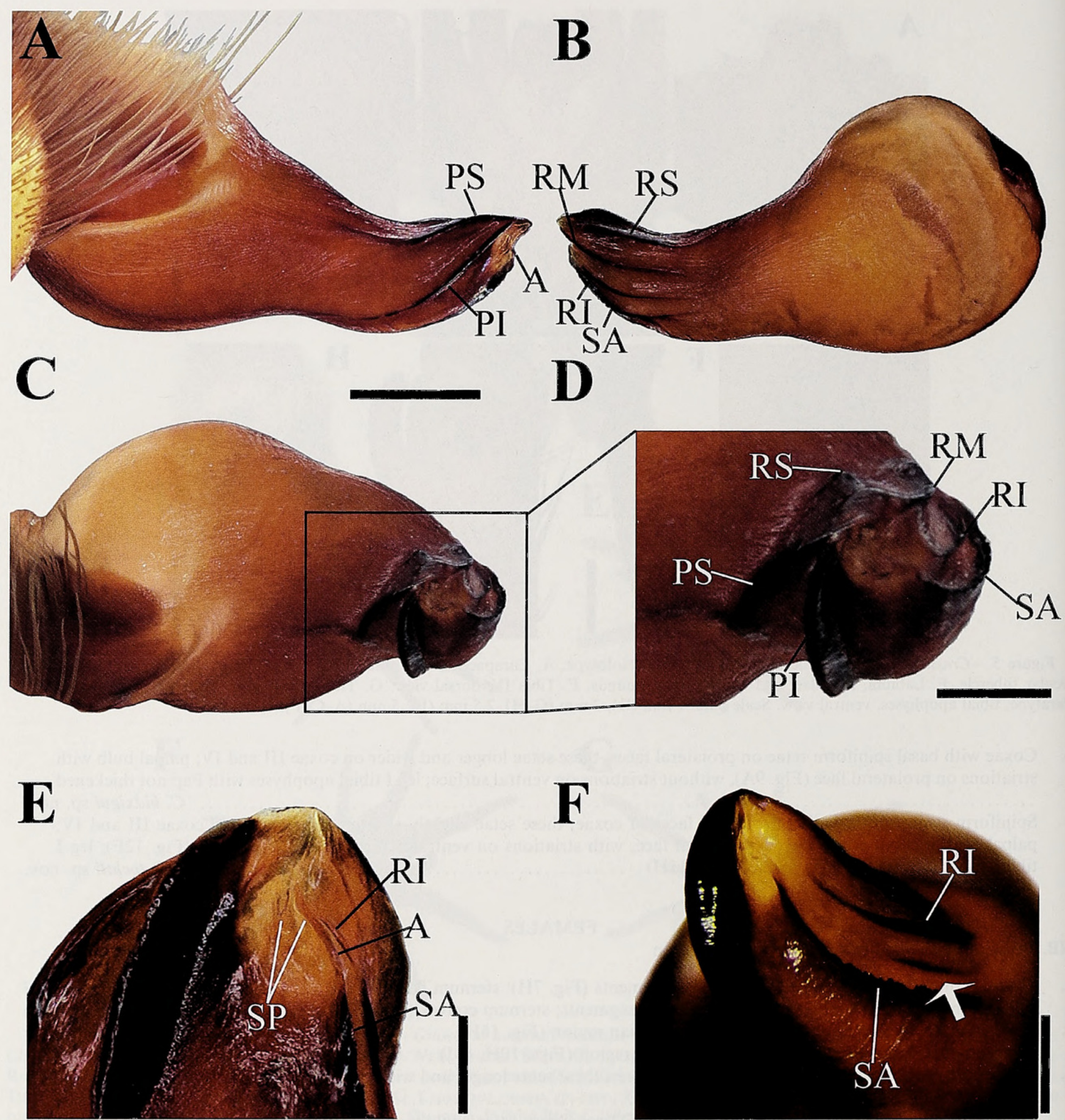

Figure 6.-Palpal bulb of male of Crassicrus lamanai. A-E. holotype: A. Prolateral view. B. Retrolateral view. C. Dorsal view. D. Detail of embolus in dorsal view. E. Apical region of embolus in ventro-prolateral view. F. Paratype, embolus in ventro-retrolateral view showing the variation of number of keels. Abbreviations: $A=$ apical keel; $P I=$ prolateral inferior keel; $P S=$ prolateral superior keel; $R I=$ retrolateral inferior keel; RM = retrolateral median keel; RS = retrolateral superior keel; $\mathrm{SA}=$ subapical keel; $\mathrm{SP}=$ spermatic pore keels. Scale bars: 0.25 (E, F), 0.5 $\mathrm{mm}(\mathrm{D}), 1 \mathrm{~mm}(\mathrm{~A}-\mathrm{C})$.

1995 (AMNH); 4 \%, same data except 9 January 1995 (AMNH).

Diagnosis.-Crassicrus lamanai can be distinguished from all other congeners except $C$. yumkimil sp. nov. by having tibia
IV thickened. Males can be separated from those of $C$. yumkimil sp. nov. by the presence of three (rather than two) keels on the retrolateral face of the embolus. Females of $C$. yumkimil sp. nov. are unknown. 

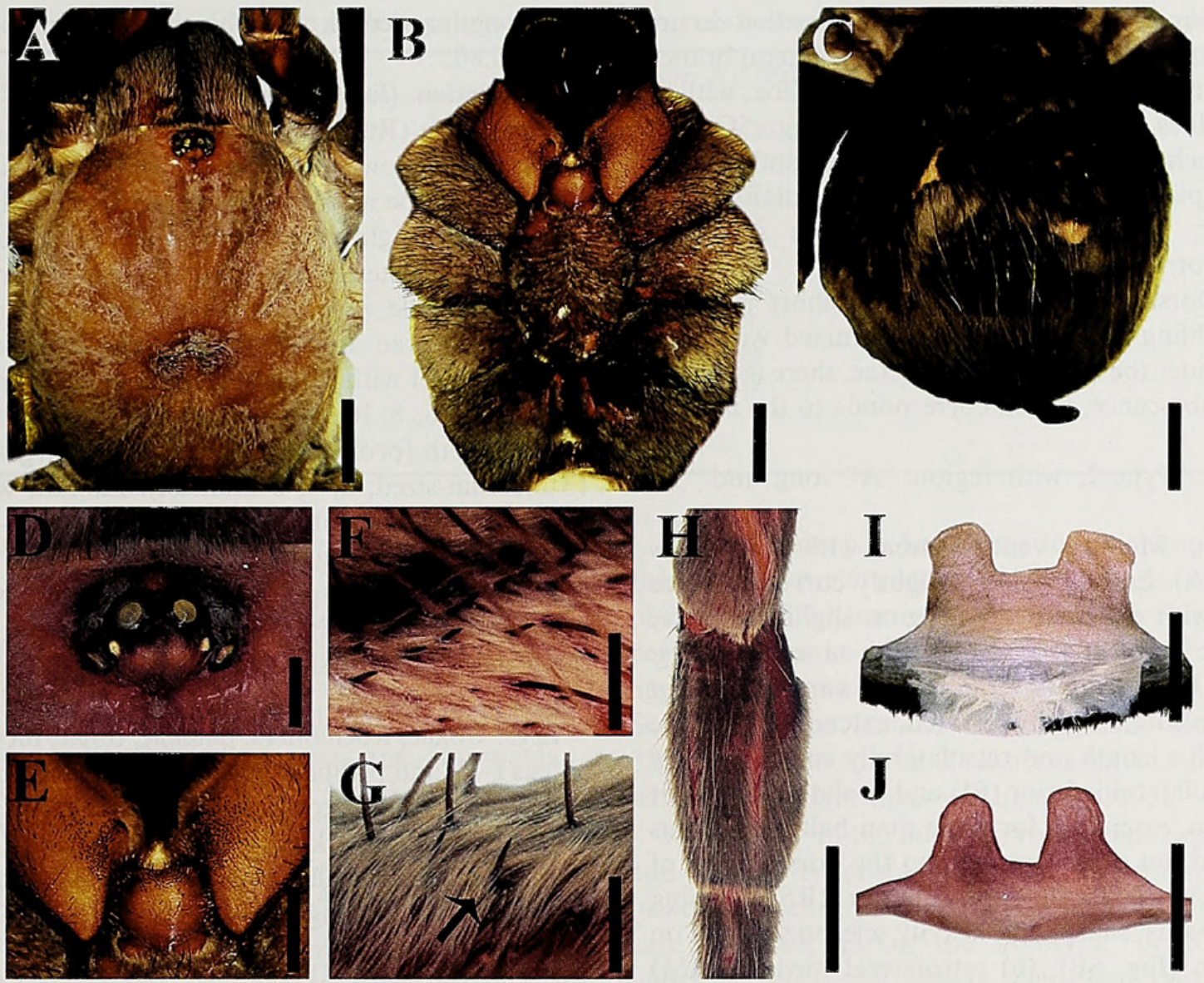

Figure 7.-Crassicrus lamanai female paratypes (AMNH): A. Carapace. B. Prosoma, ventral view. C. Abdomen, dorsal view. D. Ocular tubercle. E. Labium and maxillae. F. Conical spiniform setae on prolatero-ventral region of coxa II. G. Spiniform setae (arrow) on prolateroventral region of femur III. H. Thickened tibia IV. I, J. Spermathecae variation of (I) female of redescription and (J) female of original description. Scale bars: $0.5 \mathrm{~mm}(\mathrm{~F}, \mathrm{G}), 2 \mathrm{~mm}$ (D, I, J), $3 \mathrm{~mm}(\mathrm{E}), 5 \mathrm{~mm}$ (A-C, H).

Description (male holotype).-Prosoma: Dorsal surface covered with short, fine setae, color jet-black in life (Reichling \& West 1996), setae on carapace margin longer. Carapace semi-chordate, widest between coxae II and III (Fig. 5A). Caput slightly elevated (Fig. 5A). Fovea deep, recurved (Fig. $5 \mathrm{~A})$. Anterior eye row procurved; posterior eye row slightly recurved (Fig. 5D). AME rounded, ALE and PME oval, PLE subtriangular. Ocular tubercle wider than long; clypeus very narrow (Fig. 5D). Anterior margin of carapace covered with brown thin setae interspersed with longer and thicker setae (Fig. 5D). Chelicerae longer than wide, surface covered with coppery brown pubescence, and long thick brown setae. Prolateral furrow of chelicerae: left (damaged); right with 12 teeth (proximal to distal: 10-12 largest; 1, 3, 5, 7-9 mediumsized; 2, 4, 6 smallest). Labium wider than long, with 56 cuspules anteriorly (Fig. 5E). Labio-sternal mounds semicircular and separated (Fig. 5E). Maxillae longer than wide; left with 188 cuspules, right with 199 cuspules on baso-prolateral region (Fig. 5E). Sternum longer than wide, flat (Fig. 5B); surface covered with short, thin, grey setae, intermixed with brown setae that are longer laterally; with three pairs of oval sigilla located close to basal-retrolateral face of coxae I, II, and III; third pair largest (Fig. 5B).

Legs: All leg segments jet-black in life. Ventral surface of coxae covered with short, fine, grey setae, intermixed with brown longer setae. Coxae I-IV prolaterally covered with short cuneiform thorn-like setae, thicker ventrally. Retrolateral superior surface of maxillae and coxae I-III sparsely covered with very short spiniform setae. All other segments covered with coppery brown pubescence, intermixed with long brown setae. Femur III thickened with respect to femora I-II, IV. Tibia IV thickened with respect to tibiae I-III (Fig. 5F). Metatarsus IV longer than femur IV. Tarsal scopulae I-IV entire. Metatarsal scopulae I-III entire and IV divided by setae. Metatarsal scopulae extension: I complete, II 0.87 , III 0.65 , IV 0.18. Metatarsus I straight, when flexed touches lateral face of Rap.

Leg lateral scopulae: Pedipalp: $r$ (coxa, trochanter). Leg I: $p$ (coxa, trochanter, femur); r (coxa, trochanter). Leg II: p (coxa, trochanter, femur); $r$ (coxa, trochanter). Leg III: $p$ (coxa, trochanter); r (coxa). Leg IV: p (coxa); r (coxa, trochanter, femur).

Leg thin plumose setae: Pedipalp: $\mathrm{r}$ (trochanter, basoretrolateral face of femur). Leg I: p (trochanter, femur). Leg II: $\mathrm{p}$ (trochanter, femur).

Leg spination: Pedipalp: femur $\mathrm{p} 0-0-2 \mathrm{~d}$, tibia $\mathrm{p} 1-0-2 \mathrm{a}$. Leg I: femur p0-0-1a, tibia v0-1-1a, p0-0-2, metatarsus v0-0-1a. Leg II: femur p0-0-1d, tibia v0-2-3a(1p), p0-1-1, metatarsus v1r-1-1ap, p0-0-1v. Leg III: femur $\mathrm{d} 0-0-1 \mathrm{p}$, patella $\mathrm{r}$, tibia v1-2-1, p0-1-1, r0-1-1, metatarsus v1-2-3a(1p, 1r), p1-1-1, r0-1-1. Leg IV: tibia v1-2-3a, p-1-1-0, r0-1-1, metatarsus v13(3a), p1-1-1, r1-1-1d. 
Leg I tibial apophyses: Tibia I with two branches that do not originate from a common base (Fig. 5G, H). Prolateral branch (Pap) short, straight, digitiform, retrolateral face with a megaspine that does not protrude apically (Fig. 5G, H). Retrolateral branch (Rap) longer than Pap and curved towards it. Subapical region straight and median region slightly narrower; retroventral surface with a subapical megaspine that protrudes apically (Fig. $5 \mathrm{G}, \mathrm{H}$ ).

Opisthosoma: Dorsal surface covered with short jet-black setae in life (Reichling \& West, 1996), intermixed with long setae (Fig. 5C). Under the short jet-black setae, there is located coppery brown pubescence, which corresponds to the urticating setae.

Urticating setae: Type I, with region "A" long and "B" short.

Pedipalpal bulb: Median ventral area with a shallow depression (Fig. 6A). Embolus short, slightly curved towards retrolateral face, with dorsal median region slightly concave and distally flat (Fig. 6A, C). Embolus with nine keels (Fig. 6D, E): (1) apical keel (A) very reduced and semitransparent (Fig. 6E); (2) subapical (SA) fully serrated, extending for more than half of embolus length and retrolaterally curved distally (Fig. 6B); (3-4) prolateral inferior (PI) and prolateral superior (PS) sharp and thin, extending for more than half of embolus length, PS thin and not extending beyond the dorsal plane of embolus (Fig. 6A); (5) retrolateral superior (RS) forming dorsal edge of embolus, sharp, and heavily sclerotized only on its median portion (Fig. 6B); (6) retrolateral median (RM) thin, extending for more than half of embolus length, distally fused with PS and PI and together form the tip of embolus (Fig. 6B); (7) retrolateral inferior (RI) thicker on its median portion (Fig. 6B), distally semitransparent (Fig. 6E); (8-9) spermatic pore keels (SP) semitransparent, surrounding the seminal duct opening; the retrolateral is longer than the prolateral, curved, parallel to A keel, and it extends to the distal region of SA (Fig. 6E).

Measurements: Total length (prosoma + opisthosoma): 32.66. Leg span (measured from apex of right tarsus I to apex of right tarsus IV): 130.38. Carapace: length 15.62 , width 13.89, carapace width/length 0.89 . Ocular tubercle: height 0.78 , length 1.48, width 1.90. Eye sizes and interocular distances: AME 0.42; ALE $0.26 \times 0.50$; PME $0.17 \times 0.25$; PLE $0.34 \times 0.49$; AME-AME 0.27; AME-ALE 0.10; AMEPME 0.13, ALE-ALE 1.15, ALE-PME 0.21, PME-PME 0.98; PME-PLE 0.05; PLE-PLE 1.35; PLE-AME 0.30, PLEALE 0.24. Fovea: width 1.79. Labium: length 2.10, width 2.45 . Chelicerae: length 7.43 , width 5.36. Sternum: length 7.21, width 5.60. Leg lengths (femur, patella, tibia, metatarsus, tarsus, total): I: $15.78,7.80,12.66,13.32,8.59,58.15 ;$ II: 14.10 , $6.37,10.67,11.19,8.03,50.36$; III: $12.28,6.24,9.21,12.66$, 7.87, 48.26; IV: $15.32,6.29,13.19,18.34,9.23,62.37$. Pedipalp: $8.72,5.17,8.15,-, 3.29,25.33$. Leg formula IV, I, II, III. Leg widths: femora I-IV: $3.28,3.05,3.86,3.15$, pedipalp 2.44; patellae I-IV: $3.14,2.90,2.95,3.07$, pedipalp 2.54; tibiae I-IV: $2.33,2.55,2.55,2.81$, pedipalp 2.68 ; metatarsi I-IV 1.73, 1.66, 1.81, 1.99; tarsi I-IV: $1.90,1.66,1.61,1.44$, pedipalp 2.51 . Abdomen: length 17.04, width 13.43. Spinnerets: PMS: length 1.75, width 0.80 ; PMS-PMS: - (the spinnerets are detached from abdomen); PLS: basal 2.52, median 2.58, distal 3.01; width: $1.07,0.99$, and 0.78 respectively. Palpal bulb: length
3.88; tegulum length 1.94, height 2.02; embolus length 1.94, width 0.80 .

Description (female paratype).-Prosoma: Carapace light brown in life (Reichling \& West 1996); surface covered with short, fine, yellow setae, slightly longer marginally (Fig. 7A). Carapace shape as for holotype; caput slightly elevated (Fig. 7A). Fovea slightly recurved (Fig. 7A). Anterior eye row procurved; posterior eye row slightly recurved (Fig. 7D). Ocular tubercle wider than long; clypeus very narrow (Fig. 7D). Chelicerae longer than wide. Prolateral furrow of chelicerae: left with 13 teeth (proximal to distal: 11, 12 largest; 1, third, 3, 6, 8-10, 13 medium-sized; 2, 4, 7 smallest); right with 13 teeth (proximal to distal: 11, 12 largest; 1, 3, 5, 7-10, 13 medium-sized; 2, 4, 6 smallest). Labium wider than long, with 108 cuspules anteriorly (Fig. 7E). Labio-sternal mounds as for holotype (Fig. 7E). Maxillae longer than wide; left maxilla with 231 cuspules, right with 236 cuspules on basoprolateral region (Fig. 7E). Sternum as for holotype; with three pairs of oval sigilla located close to basal-retrolateral region of coxae I-III; third sigilla largest (Fig. 7B).

Legs: Coxae, trochanters, patellae, tibiae, metatarsi, and tarsi of legs I-IV and pedipalp light brown in life; femora of legs I-II and pedipalp dark brown; patellae, tibiae, metatarsi, and tarsi of legs III and IV dark brown, femora black (Reichling \& West 1996). Coxae ventrally covered with short, fine, grey setae interspersed with long brown setae. Coxae I-IV prolaterally covered with cuneiform thorn-like setae, which on coxae II-IV are extended ventrally (Fig. 7F). Retrolateral superior surface of maxillae and coxae I-III sparsely covered with very short spiniform setae. Proventral surfaces of femora II-IV with elongated spiniform setae (Fig. 7G). All other segments are covered with small, yellow setae interspersed with longer brown setae. Femur III thickened with respect to femora I-II, IV. Tibia III slightly thickened, tibia IV strongly thickened with respect to tibiae I-II (Fig. 7H). Femur IV slightly longer than metatarsus IV. Tarsal scopulae I-IV entire. Metatarsal scopulae I-III entire, IV divided by setae. Metatarsal scopulae extension: I complete; II 0.87 ; III 0.57 ; IV 0.12 .

Leg lateral scopulae: Pedipalp: $\mathrm{r}$ (coxa, trochanter). Leg I: $\mathrm{p}$ (coxa, trochanter, femur); r (coxa). Leg II: p (coxa, trochanter, femur); r (coxa). Leg III: p (coxa, trochanter); r (coxa). Leg IV: $\mathrm{p}$ (coxa); r (coxa, trochanter, femur).

Leg thin plumose setae: Pedipalp: $\mathrm{r}$ (trochanter, basoretrolateral face of femur). Leg I: p (trochanter, femur). Leg II: $\mathrm{p}$ (trochanter, femur).

Leg spination: Pedipalp: femur p0-0-1d, tibia v0-1p-1a, p0-1-2. Leg I: metatarsus v0-0-1a. Leg II: tibia v0-0-1ap, metatarsus v1-0-2a(1p). Leg III: tibia v0-1p-2a, p0-1-1, r0 1-1, metatarsus v2-0-2a(1p, 1r), p1-1-1, r0-1-1. Leg IV: femur d0-0-1r, tibia v0-1-2a, r1-1-1, metatarsus v15(5a), p01-1, r0-1-1.

Opisthosoma: Dorsal surface covered with short thin, brown setae, interspersed with long orange setae (Fig. 7C). Under the short brown setae, there is located dark brown pubescence, which corresponds to the urticating setae. Ventrally covered with short and long black setae.

Urticating setae: Type I, with region " $\mathrm{A}$ " long and " $\mathrm{B}$ " short.

Genitalia: Spermathecae composed of two seminal receptacles partially fused by a heavily sclerotized median region (Fig. 
Table 1.-Variation in the type specimens of Crassicrus lamanai Reichling \& West, 1996.

\begin{tabular}{lcc}
\hline \multicolumn{1}{c}{ Measurement } & o Holotype, 2 o Paratype & 5 Paratypes \\
\hline Total length & $30.24-33.39$ & $39.38-48.90$ \\
Carapace length & $15.65-17.66$ & $15.23-21.79$ \\
Carapace width & $13.89-15.75$ & $13.51-18.75$ \\
Carapace width/length & $0.89-0.97$ & $0.81-0.89$ \\
Cheliceral teeth & $11-12$ & $12-14$ \\
Labial cuspules & $24-64$ & $82-122$ \\
Maxillary cuspules & $134-199$ & $202-290$ \\
Palpal bulb length & $3.88-3.96$ & - \\
Embolus width/length & $0.51-0.61$ & - \\
Spermathecal bulbs width/length & - & $0.94-0.98$ \\
Spermathecal base width/length & - & $2.17-2.29$ \\
\hline
\end{tabular}

7I, J); each SB subquadrate, slightly wider than long (Fig. 7I, J); SS as wide as SB.

Measurements: Total length (prosoma + opisthosoma): 45.37. Leg span (measured from apex of left tarsus I to apex of left tarsus IV): 120.17. Carapace: length 21.79, width 18.75 , carapace width/length 0.83 . Ocular tubercle: height 1.03 , length 1.84 , width 2.28. Eye sizes and interocular distances: AME 0.51; ALE $0.27 \times 0.51$; PME 0.29; PLE 0.28 $\times 0.47$; AME-AME 0.32; AME-ALE 0.29; AME-PME 0.38, ALE-ALE 1.80, ALE-PME 0.53, PME-PME 1.19; PMEPLE 0.17; PLE-PLE 1.53; PLE-AME 0.49, PLE-ALE 0.38. Fovea: width 3.33. Labium: length 2.38, width 3.58 . Chelicerae: length 9.64, width 8.24. Sternum: length 10.42 , width 8.40. Leg lengths (femur, patella, tibia, metatarsus, tarsus, total): I: $14.69,9.20,10.26,9.91,6.86,50.92$; II: 13.92 , $8.34,8.54,9.26,6.89,46.95$; III: $12.52,7.62,7.93,10.33,6.44$, 44.84; IV: $15.94,8.52,11.40,15.31,7.13,58.30$. Pedipalp: $10.87,6.71,7.97,-, 7.53,33.08$. Leg widths: femora I-IV: 3.62, 3.71, 4.09, 3.83, pedipalp 2.94; patellae I-IV: 3.72, 3.80, $3.77,4.11$, pedipalp 3.03 ; tibiae I-IV: $3.04,2.82,3.46,5.32$, pedipalp 2.93; metatarsi I-IV: $2.45,2.29,2.02,2.55$; tarsi IIV: $1.95,2.13,2.00,2.04$, pedipalp 2.45. Abdomen: length 23.22, width 19.51. Spermatheca: Base: length 1.62, width 3.67; SB: length 1.09, width 1.11; SS: width 1.13; SB-SB: 1.23. Spinnerets: PMS: length 2.45, width 1.22; PMS-PMS: 0.98 ; PLS: basal 3.64, median 2.52, distal 3.50; width: 1.85 , $1.64,1.05$ respectively.

Distribution.-Known only from Belize, in the Orange Walk District (near Lamanai Forest Reserve) and Cayo District (Hummingbird Highway) (Reichling \& West 1996) (Fig. 2).
Natural history.-According to Reichling \& West (1996), this species prefers open areas such as corn and banana plantations. The burrows they found were straight and almost perpendicular to the surface. The mature males started appearing during the last days of June and were abundant in September. The females built egg sacs with approximately 350-400 eggs in March. Crassicrus lamanai is often sympatric with Brachypelma vagans (Ausserer, 1875).

Variation.- In the male paratypes the shape of the embolus and tegulum is constant, but there is variation in the number of keels present on the retrolateral face of the embolus, which can be three, as on the holotype, or four, having one underdeveloped and weakly sclerotized keel between RI and SA keels (Fig. 6F). We did not assign a name to this structure because there is no evidence of any other equivalent keel to assume primary homology. Also, because its presence is not constant in the male specimens of this species, and its low structural complexity, this keel could be a characteristic unique to certain individuals. See Tables 1-3 for details of size variation in different characters.

\section{Crassicrus bidxigui sp. nov.}

http://zoobank.org/?lsid=urn:lsid:zoobank. org:act:C6888BAF-89FB-419A-924F-2C8605903144 (Figs. 8-10)

Type material.-Holotype male. MEXICO: Oaxaca: Tolosa Donají, Matías Romero Avendaño municipality, 1-12 September 1947, B. Malkin (AMNH).

Table 2.-Variation in the lengths and widths of appendage segments for three adult males of the type series of Crassicrus lamanai Reichling \& West, 1996. Segments with the data in bold were considered as thickened.

\begin{tabular}{|c|c|c|c|c|c|}
\hline Segment & Pedipalp & Leg I & Leg II & Leg III & Leg IV \\
\hline \multicolumn{6}{|l|}{ Length } \\
\hline Femur & $8.72-9.57$ & $15.78-16.57$ & $14.10-15.03$ & $12.28-13.01$ & $15.32-16.65$ \\
\hline Patella & $5.17-5.80$ & $7.80-8.41$ & $6.37-7.72$ & $6.24-6.86$ & $6.29-7.68$ \\
\hline Tibia & $8.15-8.54$ & $12.37-13.05$ & $10.67-11.39$ & $9.63-9.71$ & $13.19-13.80$ \\
\hline Metatarsus & - & $12.83-13.41$ & $11.19-12.81$ & $12.66-13.24$ & $18.34-19.74$ \\
\hline Tarsus & $3.29-3.99$ & $8.59-9.20$ & $8.03-8.95$ & $7.87-8.35$ & $9.23-9-90$ \\
\hline Total & $25.33-27.67$ & $58.15-60.64$ & $50.36-55.90$ & $48.26-51.17$ & $62.37-67.77$ \\
\hline \multicolumn{6}{|l|}{ Width } \\
\hline Femur & $2.44-2.96$ & $3.28-3.93$ & $3.05-3.86$ & $3.86-4.16$ & $3.15-3.99$ \\
\hline Tibia & $2.68-3.12$ & $2.33-2.97$ & $2.50-2.62$ & $2.55-2.99$ & $3.46-3.77$ \\
\hline
\end{tabular}


Table 3.-Variation in the lengths and widths of appendage segments for five adult females of the type series of Crassicrus lamanai Reichling \& West, 1996. Segments with the data in bold were considered as thickened.

\begin{tabular}{|c|c|c|c|c|c|}
\hline Segment & Pedipalp & Leg I & Leg II & Leg III & Leg IV \\
\hline \multicolumn{6}{|l|}{ Length } \\
\hline Femur & $8.72-10.19$ & $11.73-14.69$ & $10.24-13.92$ & $9.59-12.52$ & $12.50-15.94$ \\
\hline Patella & $5.31-6.71$ & $6.63-9.20$ & $6.00-8.34$ & $5.55-7.62$ & $5.99-8.71$ \\
\hline Tibia & $5.51-7.97$ & $8.00-10.26$ & $7.11-8.54$ & $6.20-7.89$ & $9.53-11.68$ \\
\hline Metatarsus & - & $7.76-9.92$ & $7.84-9.47$ & $8.14-10.33$ & $11.62-15.32$ \\
\hline Tarsus & $6.26-7.53$ & $4.69-6.86$ & $4.66-6.89$ & $5.61-6.25$ & $5.73-7.13$ \\
\hline Total & $25.94-33.08$ & $38.81-49.17$ & $35.85-46.95$ & $34.09-44.84$ & $45.37-58.30$ \\
\hline \multicolumn{6}{|l|}{ Width } \\
\hline Segment & Pedipalp & Leg I & Leg II & Leg III & Leg IV \\
\hline Femur & $2.38-3.40$ & $3.18-4.03$ & $3.09-3.87$ & $3.57-4.38$ & $3.45-4.29$ \\
\hline Tibia & $2.16-3.11$ & $2.78-3.14$ & $2.27-3.02$ & $2.45-3.29$ & $4.09-5.48$ \\
\hline
\end{tabular}

Paratypes. MEXICO: Oaxaca: 1 ơ, same data as holotype except 23-20 August 1947, B. Malkin (AMNH); 3 , Palomares, Matías Romero Avendaño municipality, JulyAugust 1909, A. Petrunkevitch (AMNH); 1 \&, same data except 23 July 1909, A. Petrunkevitch (AMNH); 1 \&, outside Escuela Técnica Secundaria 104, Tolosa Donají, Matías Romero Avendaño municipality, $17.2299^{\circ} \mathrm{N}, 95.05808^{\circ} \mathrm{W}, 71$ m, 05 April 2014, C. Santibáñez, J. Cruz, D. Candia, A. Guzmán, L. Gómez (CNAN-T1094); 1 ㅇ, Piedra Blanca, San Juan Guichicovi municipality, $16.98883^{\circ} \mathrm{N}, 95.01451^{\circ} \mathrm{W}, 123$ m, 11 December 2010, S. Longhorn, J. Mendoza, E. Goyer, E. Hijmensen (CNAN-T1005). 1 \%, same data except (CNANT1006). Veracruz: 1 ๙ึ, Hacienda La Oaxaqueña, 30 km SW
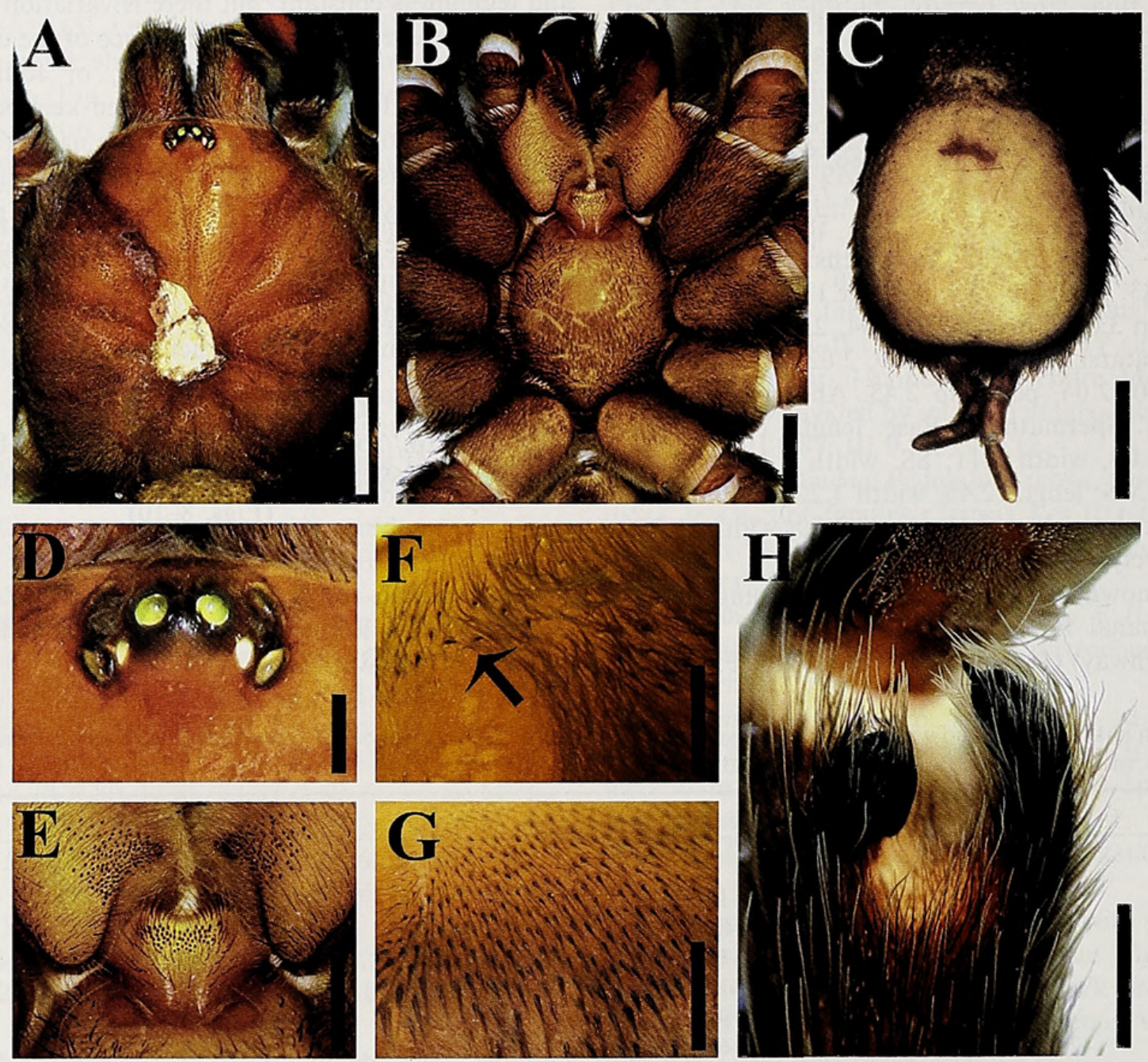

Figure 8.-Crassicrus bidxigui sp. nov. male holotype: A. Carapace. B. Prosoma, ventral view. C. Abdomen, dorsal view. D. Ocular tubercle. E. Labium, maxillae, and labio-sternal mounds. F. Spiniform setae (arrow) on retrolateral superior region of coxa I. G. Conical spiniform setae on prolateral face of coxa I. H. Tibial apophyses, ventral view. Scale bars: $0.5 \mathrm{~mm}(\mathrm{~F}-\mathrm{G}), 2 \mathrm{~mm}(\mathrm{D}, \mathrm{H}), 3 \mathrm{~mm}(\mathrm{E}), 5 \mathrm{~mm}(\mathrm{~A}-\mathrm{C})$. 

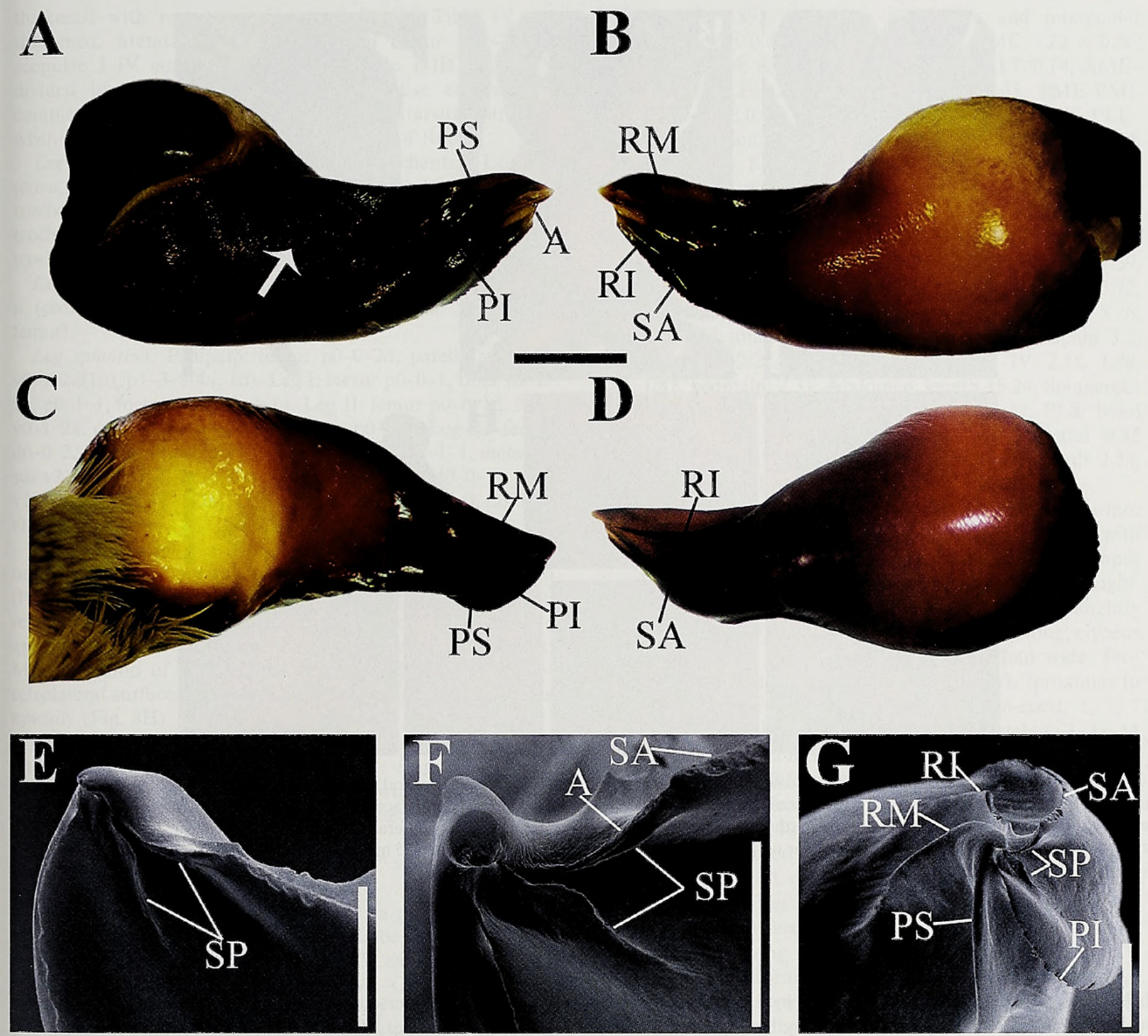

Figure 9.- Palpal bulb of males of Crassicrus bidxigui sp. nov. A-D. Holotype: A. Prolateral view. B. Retrolateral view. C. Dorsal view. D Ventral view. E-G. Apical region of embolus of male from Tolosa Donají, Matías Romero Avendaño, Oaxaca: E. prolatero-ventral view. F. Detail of spermatic pore keels. G. Antero-dorsal region. Arrows point to striations on prolateral face of the bulb. Abbreviations: A = apical keel; $\mathrm{PI}=$ prolateral inferior keel; $\mathrm{PS}=$ prolateral superior keel; $\mathrm{RI}=$ retrolateral inferior keel; $\mathrm{RM}=$ retrolateral median keel; $\mathrm{SP}=$ spermatic pore keels. Scales: $100 \mu \mathrm{m}$ (F), $250 \mu \mathrm{m}$ (E, G), $1 \mathrm{~mm}$ (A-D).

from Jesús Carranza, Coatzacoalcos river, Jesús Carranza municipality, 15 October 1939, C. M. Bogert (AMNH).

Other material examined.-MEXICO: Oaxaca: 1 o, Tolosa Donají, Matías Romero Avendaño municipality, 1-12 September 1947, B. Malkin (AMNH); 1 juvenile, Piedra Blanca, San Juan Guichicovi municipality, $16.98883^{\circ} \mathrm{N}, 95.01451^{\circ} \mathrm{W}$, 123 m, 5 April 2014, D. Candia, J. Cruz, L. Gómez, A. Guzmán, C. Santibáñez (CNAN-Ar010116).

Etymology.-The specific name is a noun in apposition in Zapotec, one of the many languages spoken in the Isthmus of
Tehuantepec region, where this species was collected "Bidxiguí" means spider in Zapotec.

Diagnosis.-Crassicrus bidxigui sp. nov. can be distinguished from all other congeners except $C$. tochtli sp. nov. and C. cocona sp. nov. by having a convex sternum (Figs. 8B, 10B). It is distinguished from C. cocona sp. nov. by lacking visible sternal sigilla, and by having proportionately shorter coxae I (Figs. 8B, 10B). It is distinguished from C. tochtli sp. nov. by having the coxal spiniform setae only on the basoprolateral face of the segment, and these setae are larger on coxae III and IV. The males can be further distinguished from 

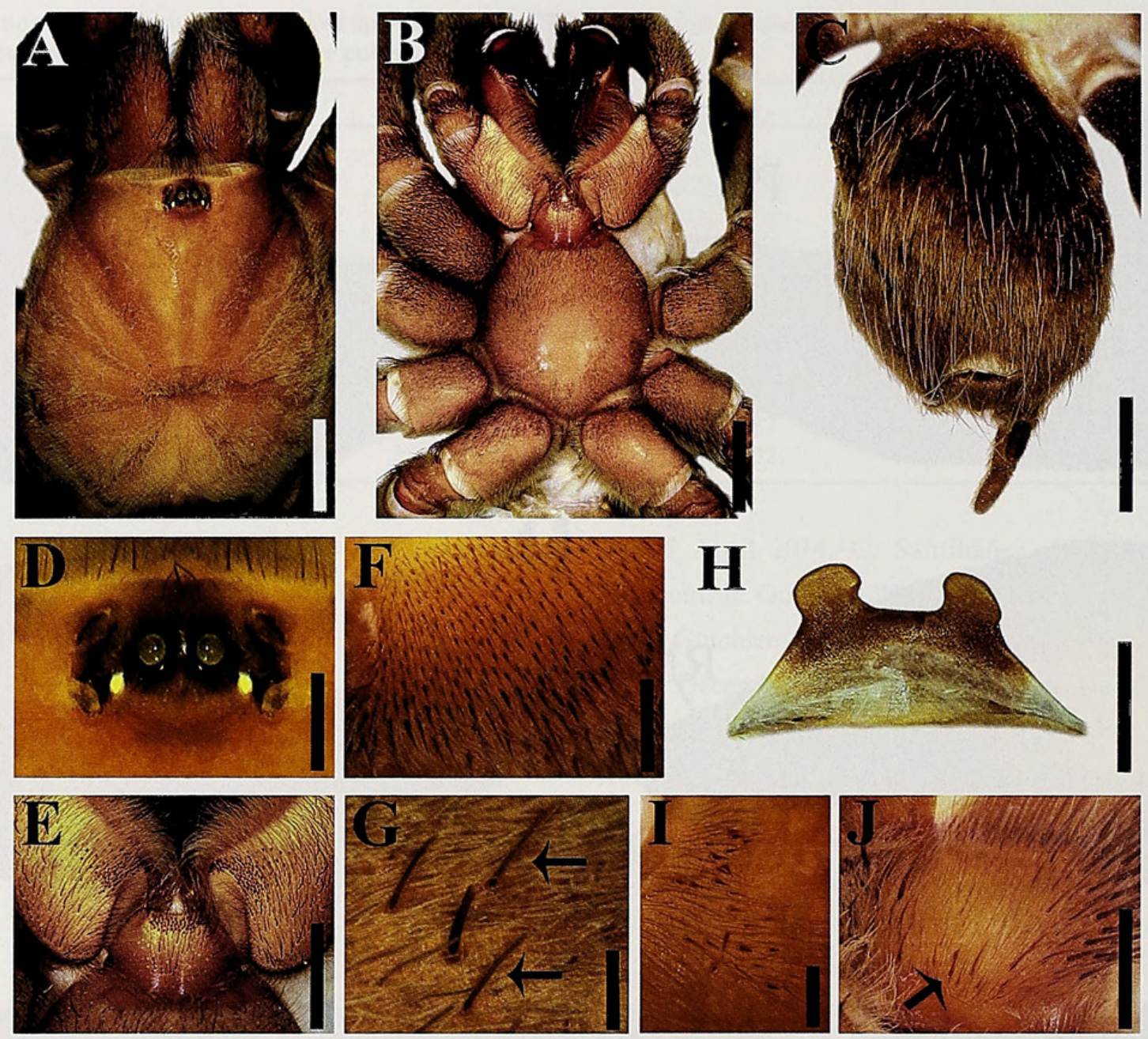

Figure 10.-Crassicrus bidxigui sp. nov. female paratype: A. Carapace. B. Prosoma, ventral view. C. Abdomen, dorsal view. D. Ocular tubercle. E. Labium, maxillae, and labio-sternal mounds. F. Spiniform setae on prolateral face of coxa I. G. Spiniform setae (arrows) on prolatero-ventral face of femur III. H. Spermathecae, dorsal view. I. Spiniform setae on retrolateral superior region of coxa I. J. Spiniform setae (arrow) protruding from the thin plumose setae on prolateral face of trochanter I. Scale bars: $0.25 \mathrm{~mm}(\mathrm{G}), 0.5 \mathrm{~mm}(\mathrm{I}), 1 \mathrm{~mm}(\mathrm{D}, \mathrm{F}, \mathrm{J}), 2 \mathrm{~mm}(\mathrm{H})$, $3 \mathrm{~mm}(\mathrm{E}), 5 \mathrm{~mm}(\mathrm{~A}-\mathrm{C})$.

those of C. tochtli sp. nov. by the presence of striations on the prolateral face of the palpal bulb, and by having the PS keel wide and extending proximally beyond the dorsal body of the embolus (Fig. 9A). The females can be further distinguished from those of $C$. tochtli sp. nov. by having a more curved edge on the median upper region of the spermathecae (Fig. 10H), by having thinner spiniform setae on the pro-ventral faces of femora II-IV, and by having femur IV longer than metatarsus IV.

Description (male holotype).-Prosoma: Dorsal surface covered with short grey setae, interspersed with thicker brown setae (Fig. 8A). Carapace semi-chordate, without pronounced boss. Caput slightly elevated (Fig. 8A). Fovea damaged. Anterior eye row procurved, posterior eye row slightly recurved (Fig. 8D). AME rounded, ALE, PME and PLE oval (Fig. 8D). Ocular tubercle wider than long; clypeus very narrow (Fig. 8D). Anterior margin of carapace covered with fine white setae interspersed with thicker yellow setae (Fig. 8D). Chelicerae longer than wide, surface covered with fine grey setae interspersed with long brown setae. Prolateral furrow of chelicerae: left: with 12 teeth (proximal to distal: 1 , 3, 10, 11 largest; 5, 7-9, 12 medium-sized; 2, 4, 6 smallest); right with 13 teeth (proximal to distal: 3, 10-12 largest; 1, 5, 79, 13 medium-sized; 2, 4, 5 smallest). Labium wider than long, with 60 cuspules anteriorly (Fig. 8E). Labio-sternal mounds semicircular and separated (Fig. 8E). Maxillae longer than wide; left with 134 cuspules, right with 154 cuspules on basoprolateral region (Fig. 8E). Sternum slightly longer than wide, convex; surface covered with short and long black setae; sigilla not visible (Fig. 8B).

Legs: Ventral surface of coxae covered with small fine grey setae, intermixed with short and long brown setae. Prolaterobasal surface of coxae I-IV covered with cuneiform thorn-like setae, thicker ventrally; these setae are noticeably larger on coxae III and IV (Fig. 8G). Retrolateral superior surface of maxillae and coxae I-III sparsely covered with very short spiniform setae (Fig. 8F). All other segments are covered with short fine grey setae and long brown setae. Femur III 
thickened with respect to femora I-II, IV. Tibia IV not thickened. Metatarsus IV longer than femur IV. Tarsal scopulae I-IV entire. Metatarsal scopulae I-III entire, IV divided by long setae. Metatarsal scopulae extension: I complete, II: 0.82; III: 0.62; IV: 0.29. Metatarsus I straight; when flexed it touches the retrolateral face of Rap.

Leg lateral scopulae: Pedipalp: $\mathrm{r}$ (coxa, trochanter). Leg I: $\mathrm{p}$ : (coxa, trochanter, femur); r (coxa, trochanter). Leg II: p (coxa, trochanter, femur); $r$ (coxa, trochanter). Leg III: p (coxa, trochanter); r (coxa). Leg IV: p (coxa, trochanter); r (coxa, trochanter, femur).

Leg thin plumose setae: Pedipalp: $r$ (coxa, trochanter). Leg I: p (coxa, trochanter, femur). Leg II: p (coxa, trochanter, femur)

Leg spination: Pedipalp: femur $\mathrm{p} 0-0-2 \mathrm{~d}$, patella $\mathrm{p} 1$, tibia v0-1-2a(1p), p1-3-8(4a, 1d). Leg I: femur p0-0-1, tibia v0-1$1 \mathrm{a}, \mathrm{p} 0-1-1$, metatarsus v0-0-1a. Leg II: femur p0-0-1d, tibia v0 $-1-2 \mathrm{a}, \mathrm{p} 1-1-1$, metatarsus v0 $0-1 \mathrm{a}, \mathrm{p} 0-0-1$. Leg III: femur d0-0-2, patella $\mathrm{r} 1$, tibia $\mathrm{v} 1-1-1, \mathrm{p} 2-3-3(2 \mathrm{a}), \mathrm{r} 1-1-1$, metatarsus v2-3-1a, p1-1-1v, r1v-1-1d. Leg IV: femur d0-0-1r, tibia v2-3-3(2ap), p1-4-3(2a), r1-1-1, metatarsus v29(6a), p0-1-1, r1-2-1.

Leg I tibial apophyses: Tibia I with two branches that do not originate from a common base (Fig. $8 \mathrm{H})$. Prolateral branch (Pap) short, slightly curved to the retrolateral branch (Rap); retrolateral face with a megaspine that does not protrude apically (Fig. 8H). Rap longer than Pap and curved towards it. Median region of Rap slightly narrower (Fig. $8 \mathrm{H}$ ); ventroretrolateral surface with a subapical megaspine that protrudes apically (Fig. $8 \mathrm{H}$ ).

Opisthosoma: Laterally covered with thin brown setae, interspersed with long, thick, yellow setae (Fig. 8C). Dorsal median region with few setae on the area where the urticating setae patch should be located; not unexpected given the age of the museum specimen (Fig. 8C).

Urticating setae: Type I, with region " $\mathrm{A}$ " very long and "B" short.

Pedipalpal bulb: Bulb with striations on prolateral face of tegulum (Fig. 9A); ventral region flat. Embolus short, slightly curved towards retrolateral face (Fig. 9E), with dorsal median region concave and distally flat (Fig. 9A-C). Embolus with eight keels (Fig. 9G): (1) apical keel (A) very reduced and semitransparent (Fig. 9D); (2) subapical (SA) fully serrated; extending for more than half of embolus length and distally is retrolaterally curved (Fig. 9A, B); (3-4) prolateral inferior (PI) and prolateral superior (PS) keels sharp and wide, extending for more than half of embolus length (Fig. 9A); PS extending beyond the dorsal plane of embolus; (5-6) retrolateral inferior (RI) and retrolateral median (RM) keels sharp and wide, extending for more than half of embolus length (Fig. 9B). RM more developed than RI; distally fused with PS and together form the tip of embolus; $(7,8)$ spermatic pore keels (SP) semitransparent, surrounding the seminal duct opening (Fig. 9D, E); the retrolateral keel is longer than the prolateral, curved, parallel to $\mathrm{A}$, and it extends to the distal region of SA (Fig. 9D-F).

Measurements: Total length (prosoma + opisthosoma): 32.79. Leg span (measured from apex of right tarsus I to apex of right tarsus IV): 126.23. Carapace: length 17.82, width 16.36, carapace width/length 0.92 . Ocular tubercle: height
0.90 , length 1.89, width 2.4. Eyes sizes and interocular distances: AME 0.32; ALE $0.40 \times 0.65$; PME $0.22 \times 0.36$; PLE $0.30 \times 0.46$; AME-AME 0.22; AME-ALE 0.14; AMEPME 0.22, ALE-ALE 1.40, ALE-PME 0.43, PME-PME 1.18; PME-PLE 0.03; PLE-PLE 1.53, PLE-AME 0.48, PLEALE 0.23. Labium: length 2.16, width 3.6. Chelicerae: length 7.24 , width 5.95 . Sternum: length 9.0 , width 8.45 . Legs length (femur, patella, tibia, metatarsus, tarsus, total): I: $15.78,8.72$, $11.84,12.24,8.27,56.85$; II: $14.83,7.23,10.82,11.14,8.05$, 52.07; III: $13.08,6.62,9.05,12.56,7.33,48.64$; IV: $15.98,7.27$, $12.79,17.9,8.78,62.72$. Pedipalp: $9.7,5.4,8.33,-, 4.16,27.59$. Leg formula: IV, I, II, III. Leg widths: femora I-IV: 3.75, 3.72, 4.2, 3.69, pedipalp: 2.91 ; patellae I-IV: $3.15,2.97,3.03,3.26$, pedipalp: 2.74 ; tibiae I-IV: $3.43,3.24,3.14,3.07$, pedipalp: 3.2 ; metatarsi I-IV 2.27, 2.23, 2.22, 2.05; tarsi I-IV: 2.18, 1.98, 1.83, 1.87, pedipalp: 2.31 . Abdomen: length 15.24 . Spinnerets: PMS: length 2.13, width 0.93; PMS-PMS: 1.17 ; PLS: basal 3.2, median 2.05, distal 3.65; width: $1.1,0.5$, and 0.85 respectively. Palpal bulb: length 4.50 ; tegulum: length 2.24 , height: 2.26 ; embolus: length: 2.26 , width: 1.30 .

Description (female paratype).-Prosoma: Dorsal surface covered with short white setae; marginally intermixed with longer setae (Fig. 10A). Carapace shape as for holotype; caput slightly elevated (Fig. 10A). Fovea not very deep, straight. Anterior eye row procurved; posterior eye row slightly recurved (Fig. 10D). Ocular tubercle wider than long; clypeus very narrow (Fig. 10D). Chelicerae longer than wide. Prolateral furrow of chelicerae: left with 14 teeth (proximal to distal: 2, 4, 11-14 largest; 5-6, 8-10 medium-sized; 1, 3, 7 smallest); right with 14 teeth (proximal to distal: 1, 3, 11-14 largest; 4, 6, 8-10, 13 medium-sized; 2, 5, 7 smallest). Labium wider than long, with 61 cuspules anteriorly (Fig. 10E). Labiosternal mounds as for holotype (Fig. 10E). Maxillae longer than wide; left with 124 cuspules, right with 128 cuspules on baso-prolateral region (Fig. 10E). Sternum as for holotype, surface covered with short grey setae and long brown setae; sigilla not visible (Fig. 10B).

Legs: Ventral surface of coxae covered with short fine white setae and brown long setae. Prolateral surface of coxae I-IV covered with cuneiform thorn-like setae, thicker ventrally (Fig. 10F). Retrolateral superior surface of maxillae and coxae I-III sparsely covered with very short spiniform setae (Fig. 10I). Prolateral and prolatero-dorsal surfaces of coxae and trochanters I-IV with long spiniform setae (Fig. 10J). Prolateroventral surface of femora II-IV covered with elongated sharp spiniform setae (Fig. 10G). All other segments are covered with short yellow setae and long brown setae. Femur III thickened with respect to femora I-II, IV. Tibia IV not thickened. Femur IV longer than metatarsus IV. Tarsal scopulae I-III entire, IV with a row of setae. Metatarsal scopulae I-III entire, IV divided by setae. Metatarsal scopulae extension: I: complete; II: 0.96; III: 0.66; IV: 0.19.

Leg lateral scopulae: Pedipalp: $r$ (coxa, trochanter). Leg I: $p$ (coxa, trochanter, femur); r (coxa, trochanter). Leg II: p (coxa, trochanter, femur); r (coxa, trochanter). Leg III: p (coxa, trochanter; r (coxa). Leg IV: p (coxa, trochanter); r (coxa, trochanter, femur).

Leg thin plumose setae: Pedipalp: $\mathrm{r}$ (trochanter). Leg I: $\mathrm{p}$ (trochanter, femur). Leg II: p (trochanter, femur). 
Table 4.-Variation in the type specimens of Crassicrus bidxigui sp. nov.

\begin{tabular}{lcc}
\hline \multicolumn{1}{c}{ Specimens } & Crassicrus bidxigui \\
\hline Total length & o Holotype and 2 o Paratypes & 6 Paratypes \\
Carapace length & $32.01-33.06$ & $29.47-40.20$ \\
Carapace width & $16.25-17.82$ & $14.68-19.88$ \\
Carapace width/length & $15.01-16.36$ & $12.46-16.65$ \\
Sternum length & $0.85-0.92$ & $0.83-0.94$ \\
Sternum width & $9.00-9.45$ & $8.10-9.45$ \\
Sternum width/length & $8.40-8.45$ & $7.95-9.50$ \\
Chelicerae teeth & $0.89-0.93$ & $12-13$ \\
Labial cuspules & $60-74$ & $11-14$ \\
Maxillary cuspules & $124-158$ \\
Bulb length & $4.30-4.50$ \\
Embolus width/length & $0.53-0.62$ \\
Spermathecae bulbs width/length & - \\
Spermathecae base width/length & - \\
\hline
\end{tabular}

Leg spination: Pedipalp: femur d0 $0-2$, tibia v0 $0-3 a(1 \mathrm{p}$, 2r), p0-2-2. Leg I: femur p0-0-1ad, metatarsus v0-0-1a. Leg II: tibia v1-0-2a(1p), p0-1-0, metatarsus v1-0-2a(1p). Leg III: femur $\mathrm{d} 0-0-1 \mathrm{r}$, tibia v0 $0-2 \mathrm{a}, \mathrm{p} 0-2-2, \mathrm{r} 0-1-0$, metatarsus v0-1-3a(1p, 2r), p1-1-1, r0-1-1. Leg IV: femur d0-0-1r, tibia v0-0-2a, p0-2-0, r1-1-1, metatarsus v15(6a), p0-1-1, r0-1-1.

Opisthosoma: Dorsal surface covered with short coppery brown setae, interspersed with long yellow setae (Fig. 10C). Under the coppery brown short setae, there is located dark brown pubescence, which corresponds to the urticating setae.

Urticating setae: Type I, with region "A" long and "B" short.

Genitalia: Spermathecae composed of two seminal receptacles partially fused by a heavily sclerotized median region with a wide, slightly curved superior border (Fig. 10H). SB wider than long; SS slightly narrower than SB.

Measurements: Total length (prosoma + opisthosoma): 37.03. Leg span (measured from apex of right tarsus I to apex of right tarsus IV): 103.81. Carapace: length 17.94, width 15.06, carapace width/length 0.84 . Ocular tubercle: height 0.83 , length 1.70 , width 2.35 . Eye sizes and interocular distances: AME 0.34; ALE $0.30 \times 0.52$; PME $0.16 \times 0.25$; PLE $0.32 \times 0.36$; AME-AME 0.38; AME-ALE 0.34; AMEPME 0.28, ALE-ALE 1.46, ALE-PME 0.43, PME-PME 1.24; PME-PLE 0.14; PLE-PLE 1.80; PLE-AME 0.62, PLEALE 0.38. Fovea: width 2.60. Labium: length 2.75, width 3.65. Chelicerae: length 9.38, width 6.73. Sternum: length 9.45, width 9.50. Legs length (femur, patella, tibia, metatarsus, tarsus, total): I: $12.44,7.22,8.91,7.71,5.8,42.08$; II: 11.35, $6.68,7.56,7.03,5.76,38.38$; III: $10.64,6.30,7.27,8.30,5.54$, 38.05; IV: $13.25,6.63,10.21,12.59,6.23,48.91$. Pedipalp: 8.95, $5.40,6.32,-, 6.64,27.31$. Leg formula: IV, I, II, III. Leg widths: femora I-IV: $3.08,3.02,3.23,3.06$, pedipalp: 2.72; patellae I-IV: $2.96,2.79,2.86,2.81$, pedipalp: 2.65 ; tibiae I-IV: $2.71,2.25,2.54,2.50$, pedipalp: 2.64; metatarsi I-IV: 2.24 , 1.94, 2.49, 2.92; tarsi I-IV: 2.32, 2.12, 2.22, 2.32, pedipalp: 2.07. Abdomen: length 19.09. Spermathecae: Base: length 1.53 , width 4.75 ; SB: length 0.74 , width 0.82 ; SS: width 0.74 ; SB-SB: 1.20 Spinnerets: PMS: length 1.97, width 1.00; PMSPMS: 1.35; PLS: basal 3.22, median 2.40, distal 3.48; width: $1.55,1.20$, and 0.92 respectively.
Distribution.- This species is found in the north-western region of the Isthmus of Tehuantepec in Oaxaca and in southern Veracruz, near the border with Oaxaca (Fig. 2).

Natural history.-Adult males were collected in August, September, and October, so the reproductive season of this species includes the rainy season. The localities where this species was found are lower than $150 \mathrm{~m}$ in elevation, and contained disturbed vegetation. Some females and juveniles were excavated from straight burrows, approximately $20 \mathrm{~cm}$ deep, and surrounded by sparse silk. In the localities where this species was found, individuals of Brachypelma sp. were also found and were more abundant.

Variation.-The number of keels is constant in all the male palpal bulbs examined $(n=6)$; however, there is variation in the general morphology of the bulbs, the development of the keels, and the depth of the striations. The width of the PS and RI keels is highly variable. In the spermathecae, the length and the width of the base are variable; however, the shape of the receptacles and BS are constant. See Tables 4-6 for details of size variation in different characters.

Crassicrus tochtli sp. nov.

http://zoobank.org/?lsid=urn:lsid:zoobank. org:act:447A8D7F-47F4-45AF-9E9C-36F258398F7E (Figs 11-13)

Type material.-Holotype male. MEXICO: Veracruz: Biological Station "Los Tuxtlas", San Andrés Tuxtla municipality, $18.58500^{\circ} \mathrm{N}, 95.0710^{\circ} \mathrm{W}, 139 \mathrm{~m}, 28$ July 2014, D. Candia (CNAN-T0898).

Paratypes. MEXICO: Veracruz: 1 , same data as holotype except 08 June 2009, J. Mendoza (CNAN-T0899). 1 same data except 28 July 2014, D. Candia (CNAN-T1090).

Other material examined.-MEXICO: Veracruz: 1 \&, 2 juveniles, same data as holotype except 29 January 2001, C. Durán (CNAN-Ar003662); 1 \&, "Castle Ranch", Coyame, Catemaco municipality, 30 January 2001, C. Durán (CNANAr003643)

Etymology.- The specific name is a noun in apposition from Nahuatl, which is the language where the name of the type 
Table 5.- Variation in the lengths and widths of appendage segments for three adult males of the type series (including the holotype) of Crassicrus bidxigui sp. nov. The segment with the data in bold was considered as thickened.

\begin{tabular}{|c|c|c|c|c|c|}
\hline Segment & Pedipalp & Leg I & Leg II & Leg III & Leg IV \\
\hline \multicolumn{6}{|l|}{ Length } \\
\hline Femur & $9.05-9.70$ & $14.90-15.78$ & $13.79-15.00$ & $12.24-13.54$ & $15.06-16.51$ \\
\hline Patella & $4.91-5.40$ & $7.44-8.72$ & $6.82-7.25$ & $6.17-6.66$ & $6.43-7.27$ \\
\hline Tibia & $7.27-8.33$ & $11.33-12.58$ & $9.96-10.91$ & $9.05-10.32$ & $12.28-13.51$ \\
\hline Metatarsus & - & $11.23-12.24$ & $10.69-11.70$ & $11.11-12.70$ & $15.43-17.92$ \\
\hline Tarsus & $3.30-4.16$ & $7.63-8.27$ & $7.33-8.16$ & $6.91-7.33$ & $7.78-8.78$ \\
\hline Total & $24.53-36.99$ & $52.53-66.90$ & $48.59-62.96$ & $45.84-59.99$ & $56.98-74.53$ \\
\hline \multicolumn{6}{|l|}{ Width } \\
\hline Femur & $2.74-2.96$ & $3.52-3.75$ & $3.50-3.72$ & $4.09-4.20$ & $1.90-2.22$ \\
\hline Tibia & $2.93-3.20$ & $2.96-3.43$ & $2.99-3.24$ & $2.90-3.14$ & $2.97-3.26$ \\
\hline
\end{tabular}

locality of "Los Tuxtlas" has its origin. The word "tochtli" means rabbit.

Diagnosis.-Crassicrus tochtli sp. nov. can be distinguished from all other congeners except $C$. bidxigui and $C$. cocona sp. nov. by the presence of a convex sternum (Figs. 11B, 13B). It is distinguished from $C$. cocona sp. nov. by the absence of visible sigilla on the sternum and by having coxae I relatively shorter (Figs. 11B, 13B). It is distinguished from $C$. bidxigui by the presence of spiniform setae fully covering the prolateral faces of coxae I-IV, which are slightly thinner and shorter on coxae III and IV. The males can be further distinguished from $C$. bidxigui by the presence of thick spiniform setae on the carapace, close to the margin (Fig. 11F), and by the presence of deep striations on the ventral region of the palpal bulb, near the embolus (Fig. 12F). The females can be further distinguished by the presence of a wide, curved superior border medially on the spermathecae (Fig. 13J), and by having metatarsus IV longer than femur IV.

Description (male holotype)-_Prosoma: carapace dark brown in life. Dorsal surface covered with short black setae (Fig. 11A). Carapace margin covered with grey setae that near the outer region are interspersed with violet setae and with thick spiniform setae, more abundant distally (Fig. 11F). Carapace semi-chordate; without pronounced boss; caput slightly elevated (Fig. 11A). Fovea deep, recurved (Fig. 11A). Anterior eye row slightly procurved; posterior eye row recurved (Fig. 11D). AME rounded, ALE and PME oval, PLE subtriangular. Ocular tubercle wider than long; clypeus very narrow (Fig. 11D). Anterior margin of carapace covered with fine, thick black setae. Chelicerae longer than wide, surface covered with grey setae; dorso-prolateral region covered with fine thin coppery setae, interspersed with thicker blue setae. Prolateral furrow of chelicerae: left with 15 teeth (proximal to distal: 13-14 largest; 1, 3, 5-6, 9-12, 15 mediumsized; 2, 4, 7-8 smallest); right with 14 teeth (proximal to distal: 3, 11-13 largest; 1, 4-5, 8-10, 14 medium-sized; 2, 6-7 smallest). Labium wider than long, surface covered with short and long dark brown setae; with 58 cuspules anteriorly (Fig. 11E). Labio-sternal mounds semicircular and separated (Fig. 11E). Maxillae longer than wide; left with 171 cuspules, right with 148 cuspules on baso-prolateral region (Fig. 11E). Sternum longer than wide, convex; surface covered with short and long black setae; sigilla not visible (Fig. 11B).

Legs: Prolateral surface of coxae I-IV covered with short cuneiform thorn-like setae, thicker ventrally (Fig. 11G). Retrolateral superior surface of maxillae and coxae I-III sparsely covered with very short spiniform setae. Coxae and trochanters with scattered violet setae dorsally. Patellae and tibiae with two longitudinal bald stripes dorsally. All other segments covered with short fine, and long black setae. Femur III thickened with respect to femora I-II, IV. Tibia IV not thickened. Metatarsus IV longer than femur IV. Tarsal scopulae I-III entire, IV with a median row of setae. Metatarsal scopulae I-III entire and IV divided by setae. Metatarsal scopulae extension: I complete, II 0.91, III 0.61, IV 0.17 . Metatarsus I straight, when flexed touches the lateral face of Rap.

Leg lateral scopulae: Pedipalp: $r$ (coxa, trochanter). Leg I: p (coxa, trochanter, femur); r (coxa). Leg II: p (coxa, trochanter,

Table 6.- Variation in the lengths and widths of appendage segments for five adult females of the type series of Crassicrus bidxigui sp. nov The segment with the data in bold was considered as thickened.

\begin{tabular}{|c|c|c|c|c|c|}
\hline Segment & Pedipalp & Leg I & Leg II & Leg III & Leg IV \\
\hline \multicolumn{6}{|l|}{ Length } \\
\hline Femur & $7.95-9.77$ & $10.90-13.90$ & $9.94-12.35$ & $9.31-11.17$ & $11.63-14.44$ \\
\hline Patella & $4.99-5.53$ & $6.29-7.99$ & $5.58-7.04$ & $5.19-6.59$ & $5.92-6.98$ \\
\hline Tibia & $5.72-6.42$ & $8.30-9.71$ & $6.93-8.65$ & $6.50-8.01$ & $8.91-10.61$ \\
\hline Metatarsus & - & $6.42-8.28$ & $6.31-8.54$ & $7.34-9.64$ & $11.04-14.23$ \\
\hline Tarsus & $5.99-7.04$ & $5.30-6.20$ & $4.87-5.97$ & $5.20-6.50$ & $5.78-7.65$ \\
\hline Total & $24.85-28.76$ & $37.21-46.08$ & $33.63-42.55$ & $33.61-41.91$ & $43.28-53.87$ \\
\hline \multicolumn{6}{|l|}{ Width } \\
\hline Femur & $2.18-2.96$ & $2.59-3.08$ & $2.73-3.09$ & $3.05-3.61$ & $2.73-2.89$ \\
\hline Tibia & $2.07-2.76$ & $2.11-2.76$ & $2.03-2.66$ & $2.19-2.83$ & $2.24-2.87$ \\
\hline
\end{tabular}



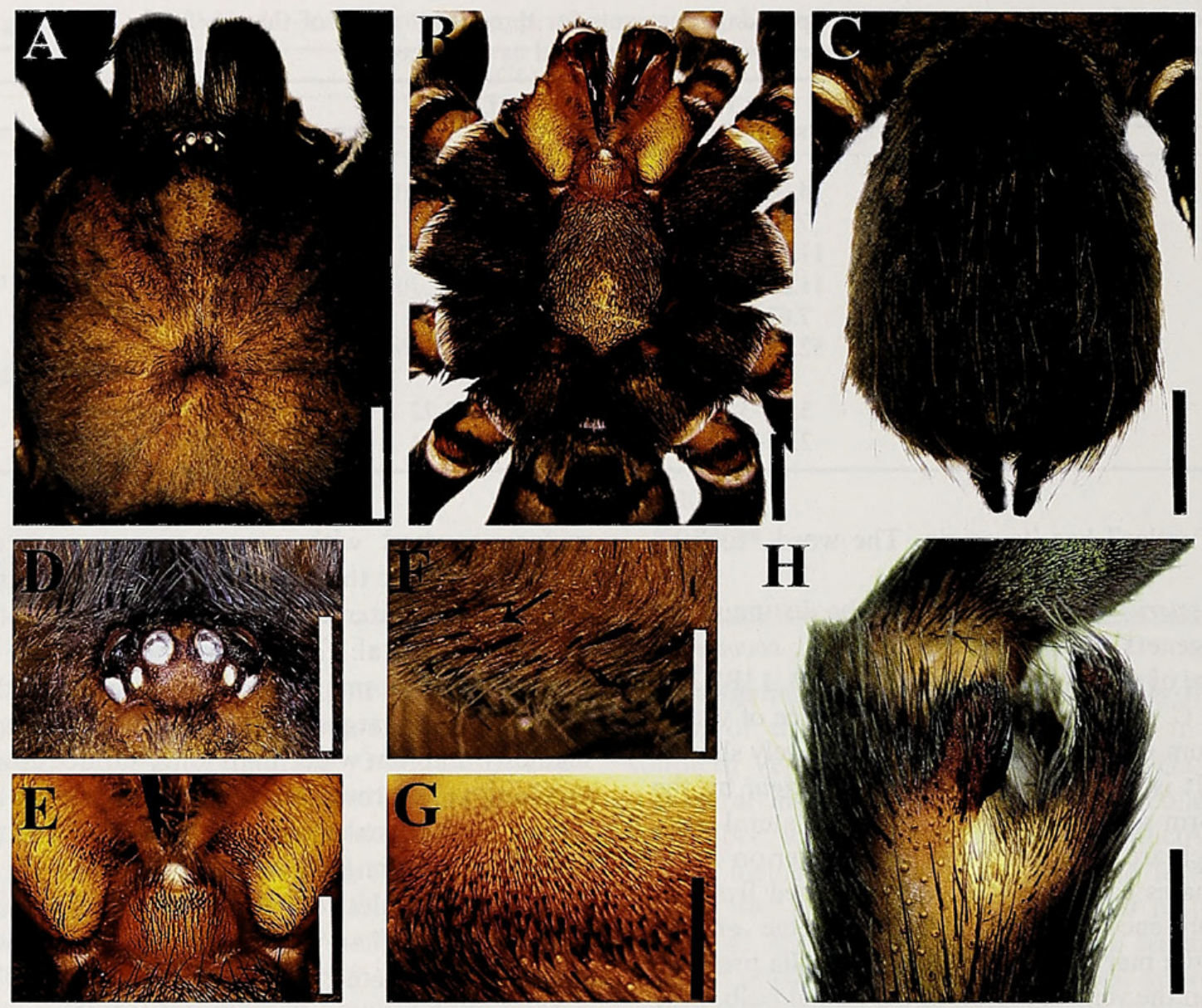

Figure 11.-Crassicrus tochtli sp. nov. male holotype: A. Carapace. B. Prosoma, ventral view. C. Abdomen, dorsal view. D. Ocular tubercle. E. Labium, maxillae, and labio-sternal mounds. F. Spiniform setae (arrow) on carapace border. G. Conical spiniform setae on prolateral face of coxa I. H. Tibial apophysis, ventral view. Scale bars: $0.5 \mathrm{~mm}(\mathrm{~F}-\mathrm{G}), 2 \mathrm{~mm}(\mathrm{D}, \mathrm{H}), 3 \mathrm{~mm}(\mathrm{E}), 5 \mathrm{~mm}(\mathrm{~A}-\mathrm{C})$.

femur); r (coxa). Leg III: p (coxa, trochanter); r (coxa). Leg IV: $\mathrm{p}$ (coxa, trochanter); r (coxa, trochanter, femur).

Leg thin plumose setae: Pedipalp: r (coxa, trochanter). Leg I: p (coxa, trochanter, femur); $r$ (coxa). Leg II: p (coxae, trochanter, femur); r (coxa).

Leg spination: Pedipalp: femur p0-0-1d, tibia p2-4-3. Leg I: femur p0-0-1, tibia v0-3(1p)-1a, p1-0-1, metatarsus v0-0-1a. Leg II: femur p0-0-1d, tibia v0-1-3a(1p) p1-1-0, metatarsus v1-0-1a. Leg III: femur p0-0-1d, r0-0-1d, tibia v2(1p)-24(2a), p1-1-1, r1-1-0, metatarsus v3-2-3a(1p, 1r), p1-1-1a, r0-1-1. Leg IV: femur r0-0-1d, patella r1, tibia v3-4-3a, p11-0, r1-1-1-1a, metatarsus v19(5a), p0-1-1, r1-1-1-1a.

Leg I tibial apophyses: Tibia I with two branches that do not originate from a common base $($ Fig. $11 \mathrm{H})$. Prolateral branch (Pap) short, thick, and straight; retrolateral face with a megaspine that does not protrude apically (Fig. 11H). Retrolateral branch (Rap) slightly longer than Pap, slightly curved towards it. Base conical, and distally digitiform; ventroretrolateral face with a subapical megaspine that protrudes apically (Fig. 11H).

Opisthosoma: Dorsally covered with short, thin black setae interspersed with long, thick, brown setae (Fig. 11C). Ventrally with short and long black setae. Under the black short setae there is located coppery brown pubescence, which corresponds to the urticating setae.

Urticating setae: Type I, with region " $\mathrm{A}$ " long, and " $\mathrm{B}$ " short.
Pedipalpal bulb: Bulb with striations on ventral face of tegulum, close to PI keel (Fig. 12A, D, F), ventral region flat (Fig. 12A, B). Embolus short, slightly curved towards retrolateral face, with dorsal median region concave and distally flat (Fig. 12A, C). Embolus with eight keels: (1) apical keel (A) very short and semitransparent (Fig. 12E); (2) subapical (SA) fully serrated, extending for more than half of embolus length and distally is retrolaterally curved (Fig. 12A, D). (3-4) prolateral inferior (PI) and prolateral superior (PS) sharp and wide, extending for more than half of embolus length (Fig. $12 \mathrm{~A})$; the distal half of PS is wide and extends beyond the dorsal plane of embolus (Fig. 12A). (5-6) retrolateral median (RM) and retrolateral inferior (RI) keels strong, slightly wider distally (Fig. 12B); extending for less than half of embolus length; RM distally fused with PS and together form the tip of embolus (Fig. 12B). (7-8) spermatic pore keels (SP) semitransparent, surrounding the seminal duct opening; the retrolateral longer than prolateral, curved, parallel to $\mathrm{A}$, and it extends to distal region of SA (Fig. 12E).

Measurements: Total length (prosoma + opisthosoma): 35.50. Leg span (measured from apex of left tarsus I to apex of left tarsus IV): 127.48. Carapace: length 18.07, width 15.69, carapace width/length 0.87 . Ocular tubercle: height 1.04, length 1.63, width 2.45. Eye sizes and interocular distances: AME 0.48 ; ALE $0.33 \times 0.63$; PME $0.18 \times 0.31$; PLE $0.36 \times$ 0.47; AME-AME 0.32; AME-ALE 0.32; AME-PME 0.14, ALE-ALE 1.42, ALE-PME 0.40, PME-PME 1.23; PME- 


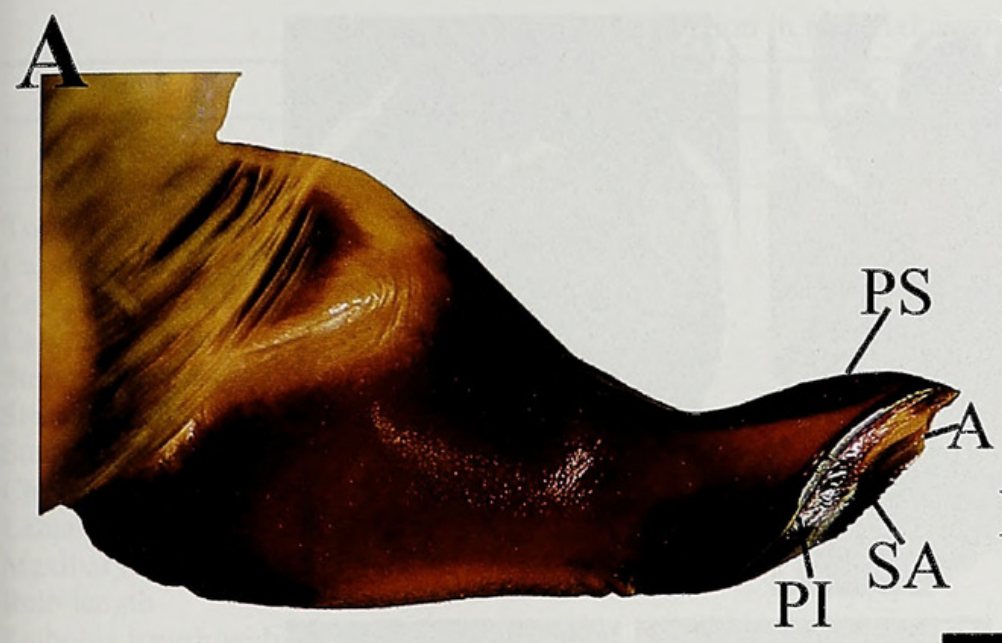

B
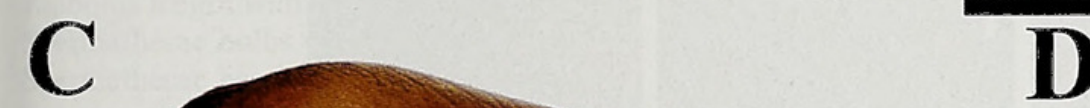

D

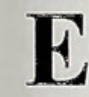

$\mathbf{E}$
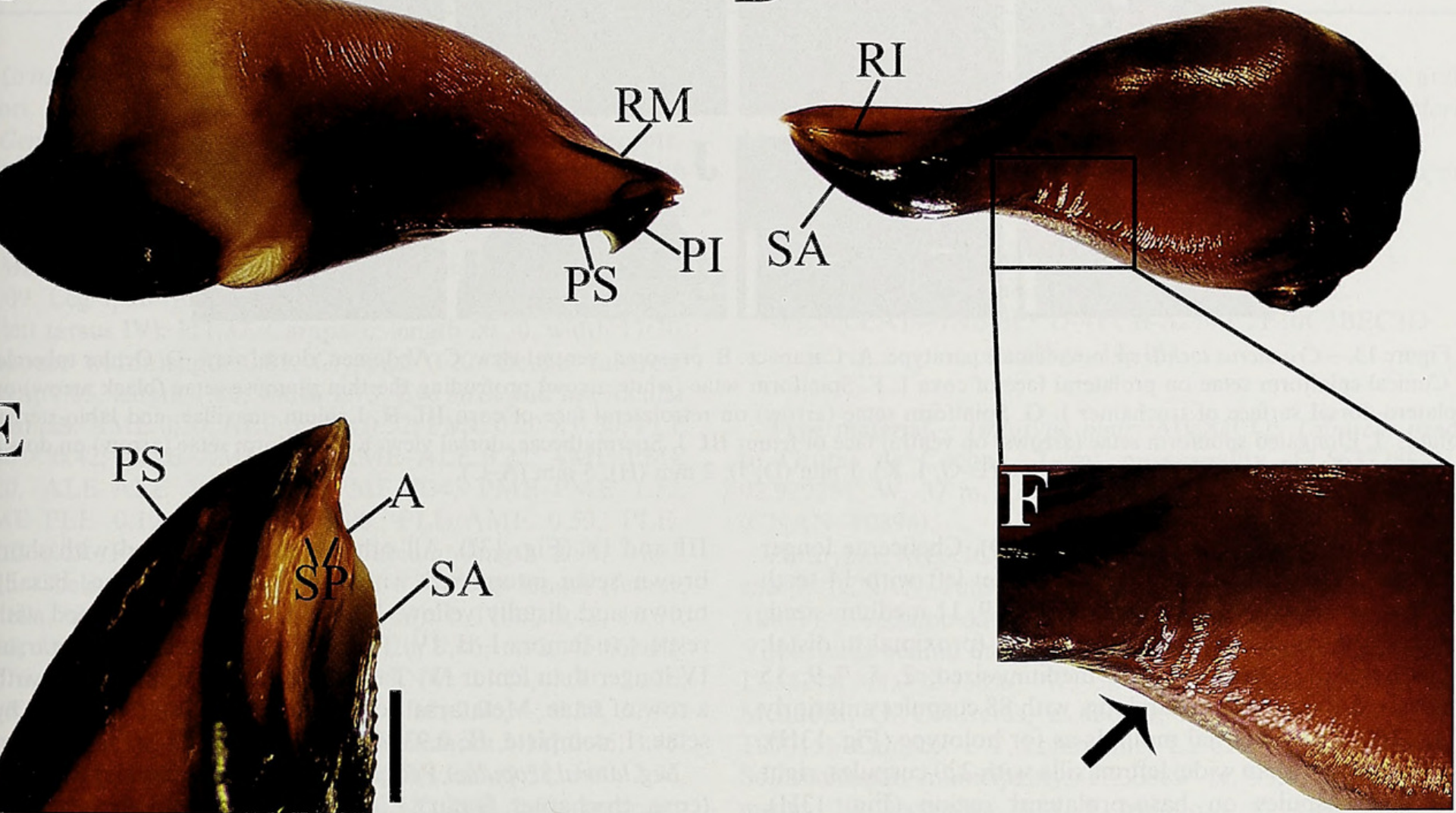

Figure 12.-Palpal bulb of male holotype of Crassicrus tochtli sp. nov.: A. Prolateral view; B, Retrolateral view. C. Dorsal view. D. Ventral view. E. Embolus apical region on prolatero-ventral view. F. Median ventral region of bulb. Arrows point to striations on ventral face of bulb. Abbreviations: $\mathrm{A}=$ apical keel; $\mathrm{PI}=$ prolateral inferior keel; PS = prolateral superior keel; $\mathrm{RI}=$ retrolateral inferior keel; RM = retrolateral median keel: $\mathrm{SP}=$ spermatic pore keel. Scale bars: $0.25 \mathrm{~mm}(\mathrm{E}), 0.50 \mathrm{~mm}(\mathrm{~F}), 1 \mathrm{~mm}(\mathrm{~A}-\mathrm{D})$.

PLE 0.14; PLE-PLE 1.66, PLE-AME 0.48, PLE-ALE 0.24. Fovea: width 2.40. Labium: length 2.67 , width 3.35 . Chelicerae: length 7.42 , width 5.79 . Sternum: length 9.50 , width 8.25 . Leg length (femur, patella, tibia, metatarsus, tarsus, total): I: $15.01,8.00,11.88,12.04,8.15,55.08$; II: $13.82,7.56,10.09$, 11.52, 7.73, 50.72; III: $12.97,6.58,9.27,12.12,6.89,47.83$; IV: 15.63, 6.77, 12.88, 17.85, 8.78, 61.91. Pedipalp: 9.79, 5.47, 8.62, -, 4.17, 27.59. Leg formula: IV, I, II, III. Width: femora I-IV: 3.57, 3.50, 3.96, 3.63, pedipalp: 2.71; patellae I-IV: $3.46,3.25$, 3.01, 3.06, pedipalp: 2.70; tibiae I-V: $3.12,2.90,2.65,3.10$, pedipalp: 3.34; metatarsi I-IV 2.09, 2.23, 1.95, 2.07; tarsi I-IV:
1.95, 1.84, 1.72, 1.58, pedipalp: 2.09. Abdomen: length 17.43. Spinnerets: PMS: length 1.96, width 0.84; PMS-PMS: 1.18 ; PLS: basal 2.80, median 1.55, distal 3.0.5; width: 1.30, 1.17, 0.87 respectively. Palpal bulb: length 4.65 ; tegulum: length 2.30, height 2.35; embolus: length 2.35, width 1.57 .

Description (female paratype CNAN-T0899).-Prosoma: Dorsal surface covered with short yellow setae, marginally intermixed with longer setae. Carapace shape same as on holotype; caput slightly elevated (Fig, 13A). Fovea not very deep, straight (Fig. 13A). Anterior eye row procurved; posterior eye row recurved (Fig. 13D). Ocular tubercle wider 

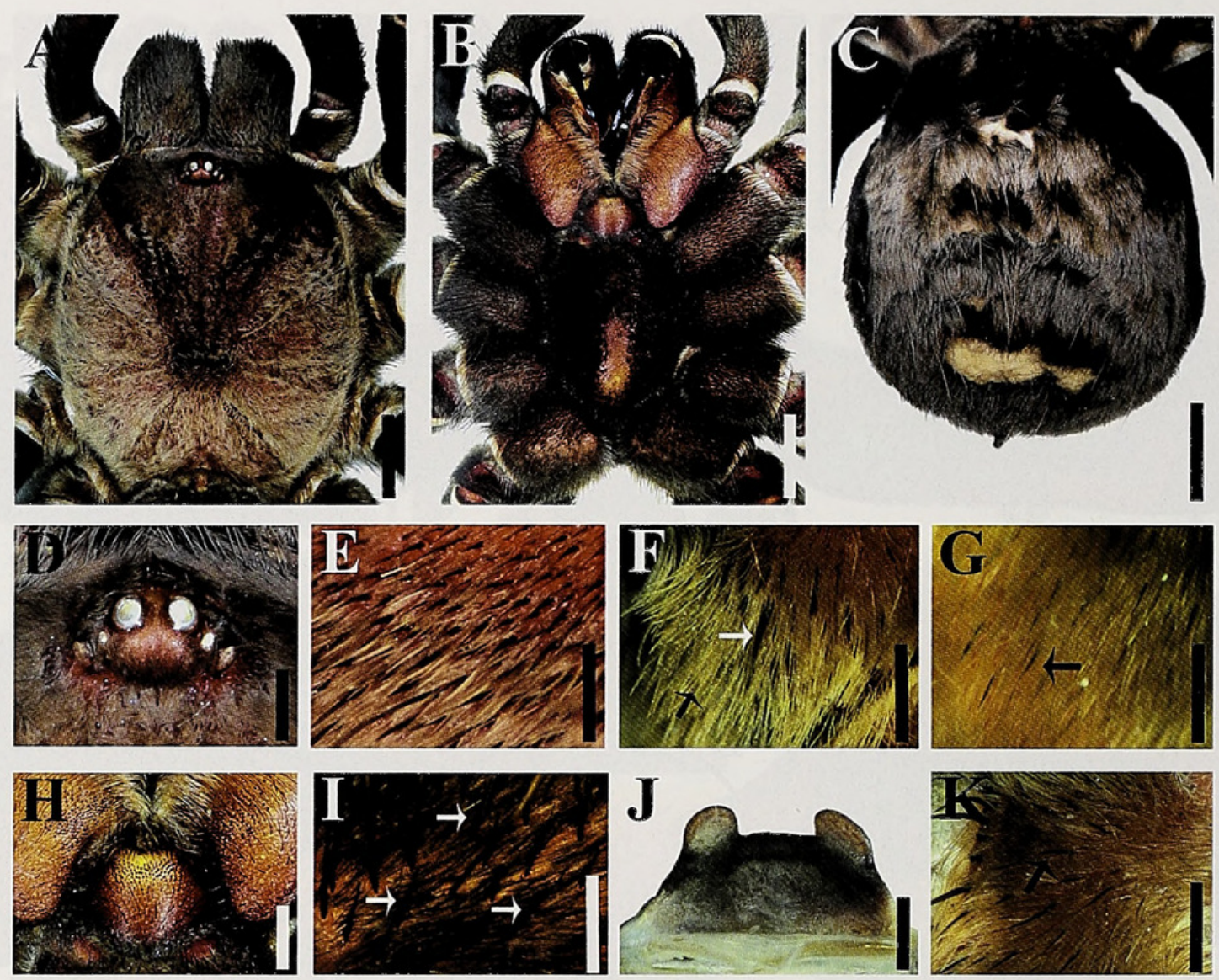

Figure 13.-Crassicrus tochtli sp. nov. female paratype: A. Carapace. B. prosoma, ventral view. C. Abdomen, dorsal view. D. Ocular tubercle. E. Conical spiniform setae on prolateral face of coxa I. F. Spiniform setae (white arrow) protruding the thin plumose setae (black arrow) on prolatero-dorsal surface of trochanter I. G. Spiniform setae (arrow) on retrolateral face of coxa III. H. Labium, maxillae, and labio-sternal mounds. I. Elongated spiniform setae (arrows) on ventral face of femur III. J. Spermathecae, dorsal view. K. Spiniform setae (arrow) on dorsal face of palpal trochanter. Scale bars: $0.5 \mathrm{~mm}(\mathrm{E}-\mathrm{G}, \mathrm{I}, \mathrm{K}), 1 \mathrm{~mm}(\mathrm{D}, \mathrm{J}), 2 \mathrm{~mm}(\mathrm{H}), 5 \mathrm{~mm}(\mathrm{~A}-\mathrm{C})$.

than long; clypeus very narrow (Fig. 13D). Chelicerae longer than wide. Prolateral furrow of chelicerae: left with 14 teeth (proximal to distal: 12-14 largest; 1, 3, 5, 9, 11 medium-sized; 2, 4, 6-8, 10 smallest); right with 15 teeth (proximal to distal: 13-14 largest; $1,3-4,6,10-12$ medium-sized; $2,5,7-9,15$ smallest). Labium wider than long, with 88 cuspules anteriorly (Fig. 13H). Labio-sternal mounds as for holotype (Fig. 13H). Maxillae longer than wide; left maxilla with 220 cuspules, right with 209 cuspules on baso-prolateral region (Fig. 13H). Sternum as for holotype, surface covered with short lightbrown setae and long dark-brown setae; sigilla not visible (Fig. 13B).

Legs: Coloration in life: coxae and trochanters I-IV brown. Patellae, tibiae, metatarsi and tarsi of legs I and II light brown with long brown setae on dorsal surfaces; patellae, tibiae, metatarsi and tarsi of legs III and IV dark brown with long reddish setae dorsally. All femora are black. Coxae I-IV prolaterally covered with cuneiform thorn-like setae, longer and slightly thicker ventrally (Fig. 13E). Retrolateral superior surface of maxillae and coxae I-III sparsely covered with very short spiniform setae, longer on coxae II and III (Fig. 13G). Dorsal surface of palpal trochanter sparsely covered with elongated spiniform setae (Fig. 13K). Prolateral and prolatero-dorsal surfaces of coxae and trochanters I-IV with thick spiniform setae (Fig. 13F). Ventro-basal surface of femora IIV with elongated sharp spiniform setae, more abundant on
III and IV (Fig. 13I). All other segments covered with short brown setae intermixed with long and thick setae, basally brown and distally yellow. Femur III slightly thickened with respect to femora I-II, IV. Tibia IV not thickened. Metatarsus IV longer than femur IV. Tarsal scopulae I-III entire, IV with a row of setae. Metatarsal scopulae I-III entire, IV divided by setae: I: complete, II: 0.93 , III: 0.65 , IV: 0.23 .

Leg lateral scopulae: Pedipalp: $\mathrm{r}$ (coxa, trochanter). Leg I: $\mathrm{p}$ (coxa, trochanter, femur); r (coxa). Leg II: p (coxa, trochanter, femur); $r$ (coxa). Leg III: $p$ (coxa, trochanter); r (coxa). Leg IV: $\mathrm{p}$ (coxa, trochanter); $\mathrm{r}$ (coxa, trochanter, femur).

Leg thin plumose setae: Pedipalp: $r$ (coxa, trochanter). Leg I: p (coxa, trochanter, femur); r (coxa, trochanter). Leg II: p (coxa, trochanter, femur).

Leg spination: Pedipalp: femur $\mathrm{p} 0-0-1 \mathrm{~d}$, tibia v0-1-3(1r), p1-2-2. Leg I: femur p0-0-1d, tibia v0-0-2(1p), metatarsus v0-0-1a. Leg II: femur p0-0-1d, tibia p0-0-3(1p), metatarsus v0 $-0-2(1 p)$. Leg III: femur $\mathrm{d} 0-0-2$, patella $1 \mathrm{r}$, tibia $\mathrm{v} 2-1-$ 3(1p), p3-1-1, r1-1-0, metatarsus v3-1-3(1p, 1r), p1-1-1, r01-1. Leg IV: tibia v2-3-3(1p), p1-1-0, r0-2-1, metatarsus v17, p0-1-0, r0-1-0.

Opisthosoma: Dorsal surface covered with short dark brown setae, interspersed with long, thick yellow setae (Fig. 13C). Under the short brown setae, there is located dark pubescence, which corresponds to the urticating setae (Fig. 13C). Ventrally covered with short and long black setae. 
Table 7.--Variation in material examined for Crassicrus tochtli sp. nov.

Crassicrus tochtli

\begin{tabular}{|c|c|c|c|}
\hline Specimens & ơ Holotype & 2 Paratypes & 29 \\
\hline Total length & 35.50 & $41.09,48.87$ & $35.75,34.29$ \\
\hline Carapace length & 18.07 & $20.30,21.16$ & $16.97,17.70$ \\
\hline Carapace width & 15.69 & $17.30,19.88$ & $14.49,15.45$ \\
\hline Carapace width/length & 0.87 & $0.85,0.94$ & $0.85,0.87$ \\
\hline Sternum length & 9.50 & $11.00,12.00$ & $9.30,10.75$ \\
\hline Sternum width & 8.25 & $8.90,9.00$ & $8.45,8.75$ \\
\hline Sternum width/length & 0.87 & $0.81,0.94$ & $0.91,0.81$ \\
\hline Chelicerae teeth & $14-15$ & $13-15$ & $13-14$ \\
\hline Labial cuspules & 58 & 88,91 & 87,81 \\
\hline Maxillary cuspules & $148-171$ & $208-220$ & $149-217$ \\
\hline Bulb length & 4.65 & - & - \\
\hline Embolus length/width & 1.50 & - & - \\
\hline Spermathecae bulbs width/length & & $1.73,1.36$ & $1.10,1.00$ \\
\hline Spermathecae base width/length & & $2.54,2.58$ & $2.53,2.34$ \\
\hline
\end{tabular}

Urticating setae: Type I, with region " $\mathrm{A}$ " long and "B" short.

Genitalia: Spermathecae composed by two seminal receptacles partially fused by a heavily sclerotized median region with a wide, curved superior border (Fig. 13J); SB wider than long; SS slightly narrower than SB. (Fig. 13J).

Measurements: Total length (prosoma + opisthosoma): 41.09. Leg span (measured from apex of left tarsus I to apex of left tarsus IV): 111.37. Carapace: length 20.30, width 17:30, carapace width/length 0.85 . Clypeus: 0.20 . Ocular tubercle: height 1.05 , length 1.80 , width 2.73 . Eye sizes and interocular distances: AME 0.50; ALE $0.34 \times 0.52$; PME $0.20 \times 0.30$; PLE $0.40 \times 0.42 ;$ AME-AME 0.22; AME-ALE 0.32; AME-PME 0.20, ALE-ALE 1.62, ALE-PME 034, PME-PME 1.52; PME-PLE 0.19; PLE-PLE 2.00; PLE-AME 0.59, PLEALE 0.29. Fovea: width 3.30. Labium: length 2.90, width 4.35. Chelicerae: length 8.52 , width 6.34. Legs length (femur, patella, tibia, metatarsus, tarsus, total): I: $14.45,8.41,10.51$, 9.84, 7.01, 50.22; II: $12.43,7.66,9.20,9.45,6.92,45.66$; III: $11.76,7.13,8.30,10.44,6.95,44.58$; IV: $14.74,7.39,11.76$, 15.37, 7.52, 56.78. Pedipalp: 10.12, 6.10, 7.96, -, 8.37, 32.55. Leg formula: IV, I, II, III. Leg widths: femora I-IV: 3.45, 3.45, 3.72, 3.57, pedipalp: 3.04; patellae I-IV: $3.23,3.22,3.00,2.96$, pedipalp: 2.74; tibiae I-IV: $2.53,2.53,2.60,2.53$, pedipalp: 2.59; metatarsi I-IV: 2.16, 2.05, 2.02, 1.94; tarsi I-IV: 1.96, 1.94, 2.02, 2.01, pedipalp: 2.31. Abdomen: length 20.79 . Spermathecae: Base: length 1.40, width 3.55; SB: length 0.56, width 0.97 ; SS width 0.97; SB-SB: 1.17. Spinnerets: PMS: length 2.00 , width 1.00; PMS-PMS: 1.50; PLS: basal 3.1, median 1.75 , distal; width: $1.20,1.10,0.65$ respectively.

Distribution.-This species is found in Veracruz, Mexico, and is known from only two localities in the municipalities of Catemaco and San Andrés Tuxtla, which are located in the central south-east of the state of Veracruz (Fig. 2).

Natural history. - The holotype male was collected as an immature; it was excavated from a perpendicular burrow approximately $25 \mathrm{~cm}$ deep and the entrance was covered with a layer of silk. It was collected in the month of July and its final molt was in mid-August, during the rainy season. The locality where the specimens were collected is in a wellconserved rainforest, in a protected area. Crassicrus tochtli is sympatric with species of the genera Brachypelma and Citharacanthus, and the arboreal species Psalmopoeus victori Mendoza, 2014.

Variation.- See Tables 7-8 for details of size variation in different characters.

Crassicrus cocona sp. nov.

http://zoobank.org/?lsid=urn:lsid:zoobank. org:act:FA144E4E-8ECD-4FCB-B250-C2F10C3BEC3D

(Figs. 14-16)

Type material.-Holotype male. MEXICO: Tabasco: road to Grutas de Coconá, Teapa municipality, $17.563728^{\circ} \mathrm{N}$, 92.929281 ${ }^{\circ} \mathrm{W}, 37 \mathrm{~m}, 13$ April 2014, D. Candia, B. Ramírez (CNAN-T0894).

Paratypes. MEXICO: Tabasco: 1 \%, same data as holotype except (CNAN-T0895). 1 $\uparrow$, same data except (CNANT0896). 1 ㅇ, same data as holotype except (CNAN-T0897). 1 O, pastures behind San Felipe Cemetery, Teapa municipality, $17.54218^{\circ} \mathrm{N}, 92.95899^{\circ} \mathrm{W}, 53 \mathrm{~m}, 26$ December 2011, J. Mendoza, G. Contreras, E. Goyer, E. Hijmensen (CNANT01015). Chiapas: 1 \&, community La Carretera, La Unión, Solosuchiapan municipality, $17.38889^{\circ} \mathrm{N}, 93.02342^{\circ} \mathrm{W}, 231$ msnm, 19 December 2011, J. Mendoza, G. Contreras, E. Hijmensen, E. Goyer (CNAN-T01016). 1 ㅇ, same data except (IBSP 166991).

Other material examined.-MEXICO: Chiapas: $1 \delta$ subadult, community La Carretera, La Unión, Solosuchiapan municipality, $17.38889^{\circ} \mathrm{N}, 93.02342^{\circ} \mathrm{W}, 231 \mathrm{msnm}, 19$ December 2011, J. Mendoza, G. Contreras, E. Hijmensen, E. Goyer (CNAN-Ar004153).

Etymology.- The specific name is a noun in apposition from the Zoque language, where the name of Cocona Caves has its origin. The word "coconá" means "deep water".

Diagnosis.-Crassicrus cocona sp. nov. can be distinguished from all other congeners except $C$. bidxigui and $C$. tochtli by the presence of a convex sternum (Figs. 14B, 16B). It is distinguished from $C$. bidxigui and $C$. tochtli by the presence of relatively longer coxae, and poorly developed spiniform setae prolaterally on coxae III-IV (Fig. 16G). The males can be further distinguished from $C$. tochtli by the presence of 
Table 8.-Variation in the lengths and widths of appendage segments for four adult females of Crassicrus tochtli sp. nov. (including paratypes). The segment with the data in bold was considered as thickened.

\begin{tabular}{|c|c|c|c|c|c|}
\hline Segment & Pedipalp & Leg I & Leg II & Leg III & Leg IV \\
\hline \multicolumn{6}{|l|}{ Length } \\
\hline Femur & $8.97-10.81$ & $12.60-15.34$ & $11.44-14.03$ & $10.45-13.35$ & $13.25-16.98$ \\
\hline Patella & $5.12-6.99$ & $7.91-9.17$ & $6.40-8.66$ & $5.73-8.05$ & $6.13-8.14$ \\
\hline Tibia & $6.90-7.96$ & $9.21-11.33$ & $8.33-9.89$ & $7.95-8.98$ & $10.39-12.80$ \\
\hline Metatarsus & - & $7.89-10.33$ & $7.54-10.31$ & $8.99-11.65$ & $13.46-17.35$ \\
\hline Tarsus & $6.99-9.11$ & $6.36-8.48$ & $5.90-7.94$ & $5.67-7.37$ & $6.71-8.71$ \\
\hline Total & $27.98-34.86$ & $43.97-54.65$ & $39.61-50.83$ & $38.79-49.40$ & $49.94-63.98$ \\
\hline \multicolumn{6}{|l|}{ Width } \\
\hline Femur & $2.53-3.04$ & $3.16-3.51$ & $3.17-3.45$ & $3.34-3.75$ & $3.24-3.57$ \\
\hline Tibia & $2.53-2.83$ & $2.53-2.87$ & $2.27-2.84$ & $2.44-2.96$ & $2.46-2.91$ \\
\hline
\end{tabular}

striations on the prolateral face of the palpal bulb (Fig. 15A); and from $C$. bidxigui by having the region above RM very concave and wide (Fig. 15B), and by having tiny denticles on the proximal region of RI (Fig. 15F). Females can be further distinguished from $C$. tochtli and C. bidxigui by the presence of longer and thicker spiniform setae on proventral faces of femora II-IV (Fig. 16I).

Description (male holotype).-Prosoma: Carapace brown in life, with iridescent coppery brown setae on ocular tubercle; cephalic region darker. Dorsal surface covered with short grey setae and black thin setae; the grooves on thoracic region covered with coppery brown setae. Carapace margins covered with black and coppery brown setae, interspersed with violet setae on outer region; close to border are long, thin, sharp spiniform setae. Carapace semi-chordate; without pronounced boss. Caput slightly elevated (Fig. 14A). Fovea deep and slightly recurved (Fig. 14A). Anterior eye row procurved; posterior eye row recurved (Fig. 14D). AME rounded, ALE and PME oval, PLE subtriangular. Ocular tubercle wider than long; clypeus very narrow (Fig. 14D). Anterior margin of carapace covered with thin yellow setae and with thick setae basally black and distally yellow. Chelicerae longer than wide, surface covered with white setae, interspersed with iridescent coppery brown and black setae; dorso-prolateral region covered with long setae basally brown and distally yellow. Prolateral furrow of chelicerae: left with 14 teeth (proximal to distal: 4, 11-13 largest; 1, 6, 8-10, 14 medium-sized; 2, 3, 5, 7 smallest); right with 15 teeth (proximal to distal: $3,12-14$ largest; 1, 7, 9-11, 15 medium-sized; 2, 4-6, 8 smallest). Labium wider than long, surface covered with short and long brown setae; with 53 cuspules anteriorly (Fig. 14E). Labiosternal semicircular and separated (Fig. 14E). Maxillae longer than wide; left with 162 cuspules, right with 175 cuspules on baso-prolateral region (Fig. 14E). Sternum longer than wide, convex; surface covered with short and long black setae; with three pairs of oval sigilla located close to baso-retrolateral face of coxae I-III; third pair largest and located close to sternum edge (Fig. 14B).

Legs: Dorsal surface of coxae and trochanters covered with dark brown and violet setae. Coxae I-IV prolaterally covered with cuneiform thorn-like setae, thicker ventrally (Fig. 14H). Retrolateral superior surface of maxillae and coxae I-III sparsely covered with very short spiniform setae. Femora covered with fine black tiny setae and violet setae. All other segments are covered by short and long fine black setae.
Femur III thickened with respect to femora I-II, IV. Tibia IV not thickened. Metatarsus IV longer than femur IV. Tarsal scopulae I-IV entire. Metatarsal scopulae I-III entire, IV divided by long setae. Metatarsal scopulae extension: I complete, II complete, III 0.65, IV 0.18. Metatarsus I straight, when flexed touches the lateral face of Rap.

Leg lateral scopulae: Pedipalp: $r$ (coxa, trochanter). Leg. I: $p$ (coxa, trochanter, femur); r (coxa, trochanter). Leg II: p (coxa, trochanter, femur); r (coxa). Leg III: p (coxa); r (coxa, trochanter). Leg IV: $p$ (coxa); $r$ (coxa, trochanter, femur).

Leg thin plumose setae: Pedipalp: $r$ (coxa, trochanter). Leg I: p (coxa, trochanter, femur); r (coxa, trochanter). Leg II: $p$ (coxa, trochanter, femur); r (coxa, trochanter).

Leg spination: Pedipalp: femur p0-0-1, tibia v0-0-2(1ap), p0-2-2. Leg I: femur p0-0-1, tibia v0-0-1a, p1-1-0, metatarsus v0 $0-1 a$. Leg II: femur p0-0-1d, tibia v0-1$3 a(1 p), p 1-1-1$, metatarsus v0-1-2a, p1-1-0. Leg III: femur d0 $-0-2$, patella $1 \mathrm{r}$, tibia v2-3-3a(1p), p1-1-1, r1-1-1, metatarsus v2-0-2a(1p), pl-2-1, r1-0-1. Leg IV: femur d0$0-1 \mathrm{r}$, patella $\mathrm{r} 1$, tibia $\mathrm{v} 1-1-2-1, \mathrm{p} 1-1-0, \mathrm{r} 1-1-1-1$, metatarsus v30(6a), p1-1-1-1, r1-1-0-0.

Leg I tibial apophyses: Tibia I with two branches that do not originate from a common base (Fig. 14G). Prolateral branch (Pap) elongated, slightly curved towards the retrolateral branch (Rap), retrolateral face with a megaspine that does not protrude apically. Rap slightly longer than Pap, almost straight, on its apical portion is curved towards Pap (Fig. 14G). Base subconical; ventro-retrolateral region with a subapical megaspine that protrudes apically.

Opisthosoma: Dorsal surface covered with short, thin black setae interspersed with long, thick orange setae (Fig. 14C). Under the short black setae, there is located dark pubescence, which corresponds to the urticating setae. Ventrally covered with short and long black setae.

Urticating setae: Type I, with region "A" long and "B" short (Fig. 14F). The urticating setae on MM region are modified, retaining the helicoidally main barbs (Fig. 14I), but with region "A" very elongated and "B" reduced (Fig. 14F); region of reversible barbs very reduced (Fig. 14J), difficult to observe and can give the setae an appearance of urticating setae type III.

Pedipalpal bulb: Bulb with striations on prolateral face of tegulum; ventral region flat (Fig. 15A). Embolus short, slightly curved towards retrolateral face, with dorsal median region concave and distally flat (Fig. 15A, C). Embolus with eight 

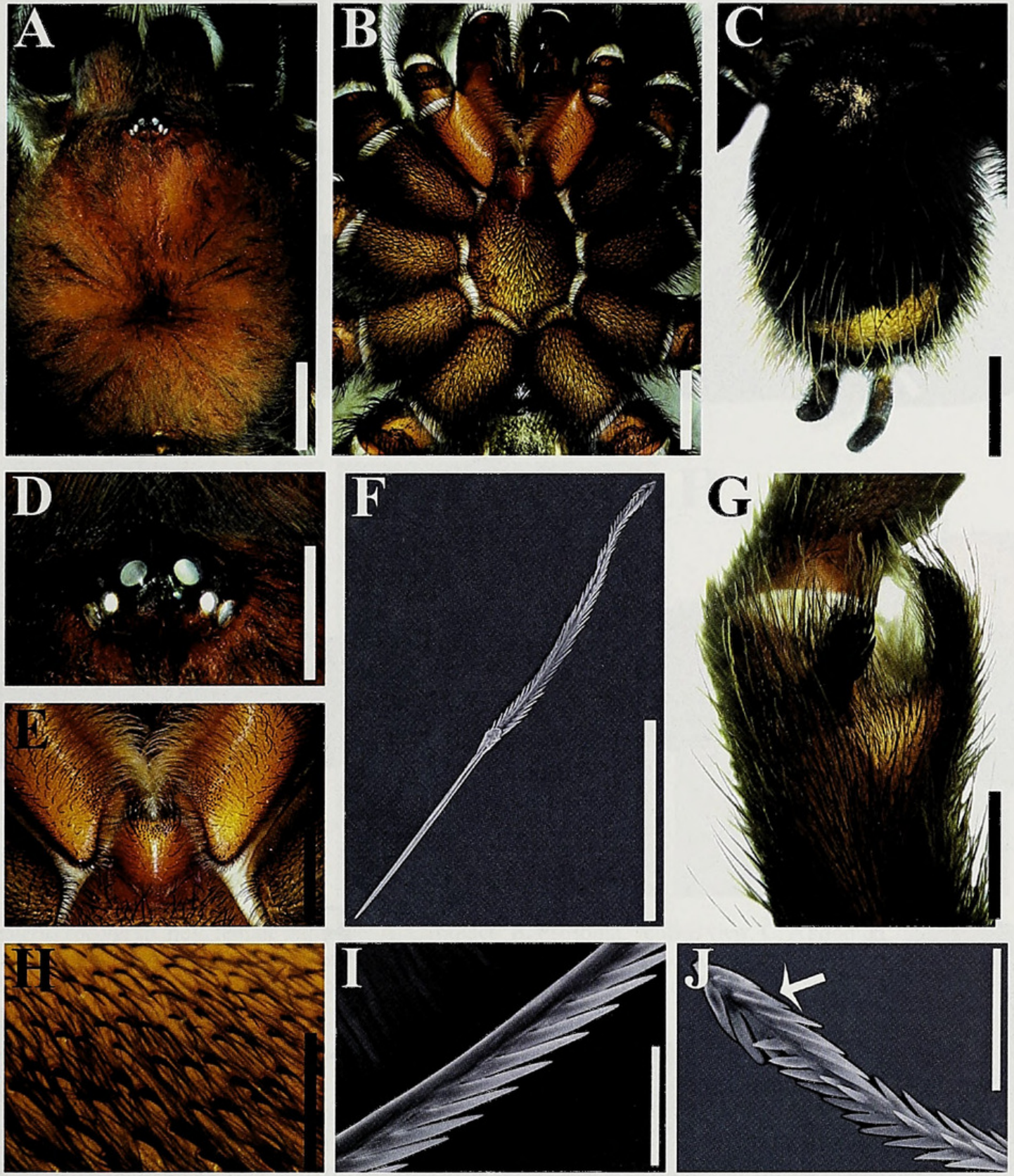

Figure 14.-Crassicrus cocona sp. nov. male holotype: A. Carapace. B. Prosoma, ventral view. C. Abdomen, dorsal view. D. Ocular tubercle. E. Labium, maxillae, and labio-sternal mounds. F. Urticating setae type I. G. Tibial apophysis, ventral view. H. Conical spiniform setae on prolateral face of coxae. I, J. Modified urticating setae type I on median region of abdomen showing (I) detail of main barbs and (J) detail of reversed barbs (arrow). Scale bars: $20 \mu \mathrm{m}$ (I), $30 \mu \mathrm{m}$ (J), $200 \mu \mathrm{m}(\mathrm{F}), 0.25(\mathrm{H}), 2 \mathrm{~mm}(\mathrm{D}-\mathrm{E}), 5 \mathrm{~mm}(\mathrm{~A}-\mathrm{C}, \mathrm{G})$.

keels: (1) apical keel (A) very reduced and semitransparent (Fig. 15E); (2) subapical keel (SA) fully serrated, extending for more than half of embolus length, distally is retrolaterally curved (Fig. 15B, D); (3-4) prolateral inferior (PI) and prolateral superior (PS) keels sharp and wide, extending for more than half of embolus length (Fig. 15A), PS pronounced and extending beyond dorsal plane of embolus (Fig. 15A); (56) retrolateral inferior (RI) and retrolateral median (RM) strong, extending for less than half of embolus length (Fig. 15B), RM distally fused with PS and together form the tip of embolus, RI with small denticles on proximal region (Fig. 15F); (7-8) spermatic pore keels (SP) semitransparent, surrounding the seminal duct opening; the retrolateral is two times longer than prolateral, curved, parallel to $\mathrm{A}$, and it extends to the distal region of SA (Fig. 15E).

Measurements: Total length (prosoma + opisthosoma): 36.60. Leg span (measured from apex of left tarsus I to apex of left tarsus IV): 144.34. Carapace: length 18.62, width 17.23, carapace width/length 0.93 . Ocular tubercle: height 1.12 , length 1.83, width 2.40. Eye sizes and interocular distances: AME 0.58; ALE $0.42 \times 0.50$; PME $0.25 \times 0.36$; PLE $0.31 \times$ 0.46; AME-AME 0.34; AME-ALE 0.22; AME-PME 0.10, ALE-ALE 1.54, ALE-PME 0.44, PME-PME 1.20; PMEPLE 0.07; PLE-PLE 1.66, PLE-AME 0.42, PLE-ALE 0.38 Fovea: width 2.40 . Labium: length 2.10 , width 3.10 . Chelicerae: length 9.16, width 6.73 . Sternum: length 8.20 , width 7.30 . Legs length (femur, patella, tibia, metatarsus, tarsus, total): I: 
A

B
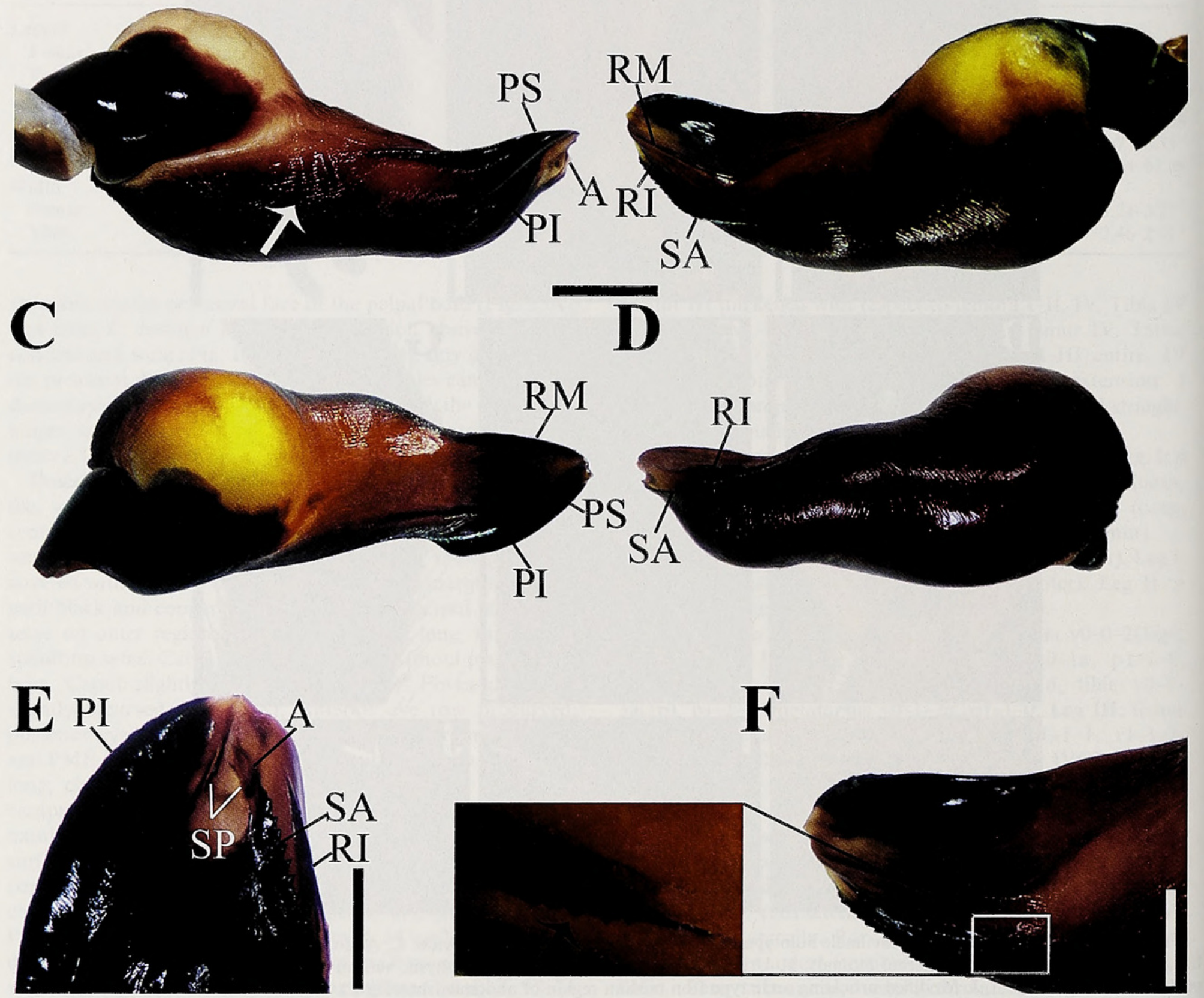

Figure 15.-Palpal bulb of male holotype of Crassicrus cocona sp. nov.: A. Prolateral view. B. Retrolateral view. C. Dorsal view. D. Ventral view. E. Embolus apical region on prolatero-ventral view. F. Detail of retrolateral keels. White arrow points to striations on prolateral face of bulb; black arrow points to denticles on posterior portion of retrolateral inferior keel. Abbreviations: $\mathrm{A}=\mathrm{apical}$ keel; PI = prolateral inferior keel; PS = prolateral superior keel; RI = retrolateral inferior keel; RM = retrolateral median keel. SP: spermatic pore keels. Scale bars: 0.5 mm (E-F), $1 \mathrm{~mm}(\mathrm{~A}-\mathrm{D})$.

$17.78,9.62,14.55,14.48,10.45,66.88 ;$ II: $16.81,9.06,12.91$, $13.85,10.44,63.07$; III: $15.11,7.70,11.38,14.83,9.72,58.74$ IV: $18.40,8.53,15.21,21.39,9.85,73.38$. Pedipalp: $10.57,6.71$, 9.43 -, 4.41, 31.12. Leg formula: IV, I, II, III. Leg widths: femora I-IV: 4.03, 3.99, 4.68, 3.92, pedipalp: 3.34; patellae IIV: $3.62,3.54,3.51,3.49$, pedipalp: 2.70 ; tibiae I-IV: 3.32 , $3.17,3.09,3.38$, pedipalp: 3.40 ; metatarsi I-IV: $2.21,2.50$, $2.33,2.27$; tarsi I-IV: $1.91,1.70,1.77,1.70$, pedipalp: 2.71 . Abdomen: length 18.04. Spinnerets: PMS: length 2.15, width 0.90; PMS-PMS: 1.00; PLS: basal 3.55, median 3.05, distal
4.35; width: $1.42,1.25$, and 0.90 respectively. Palpal bulb: length 4.65 ; tegulum length 2.30 , height 2.35 ; embolus length 2.35 , width 1.23 .

Description (female paratype CNAN-T0895). - Prosoma: Carapace brown in life, cephalic region darker. Dorsal surface covered with short and long thin yellow setae (Fig. 16A). Anterior margin of carapace covered with long brown setae, intermixed with long reddish setae. Carapace shape as for holotype; caput slightly elevated (Fig. 16A). Fovea not very deep, slightly procurved (Fig. 16A). Anterior eye row 

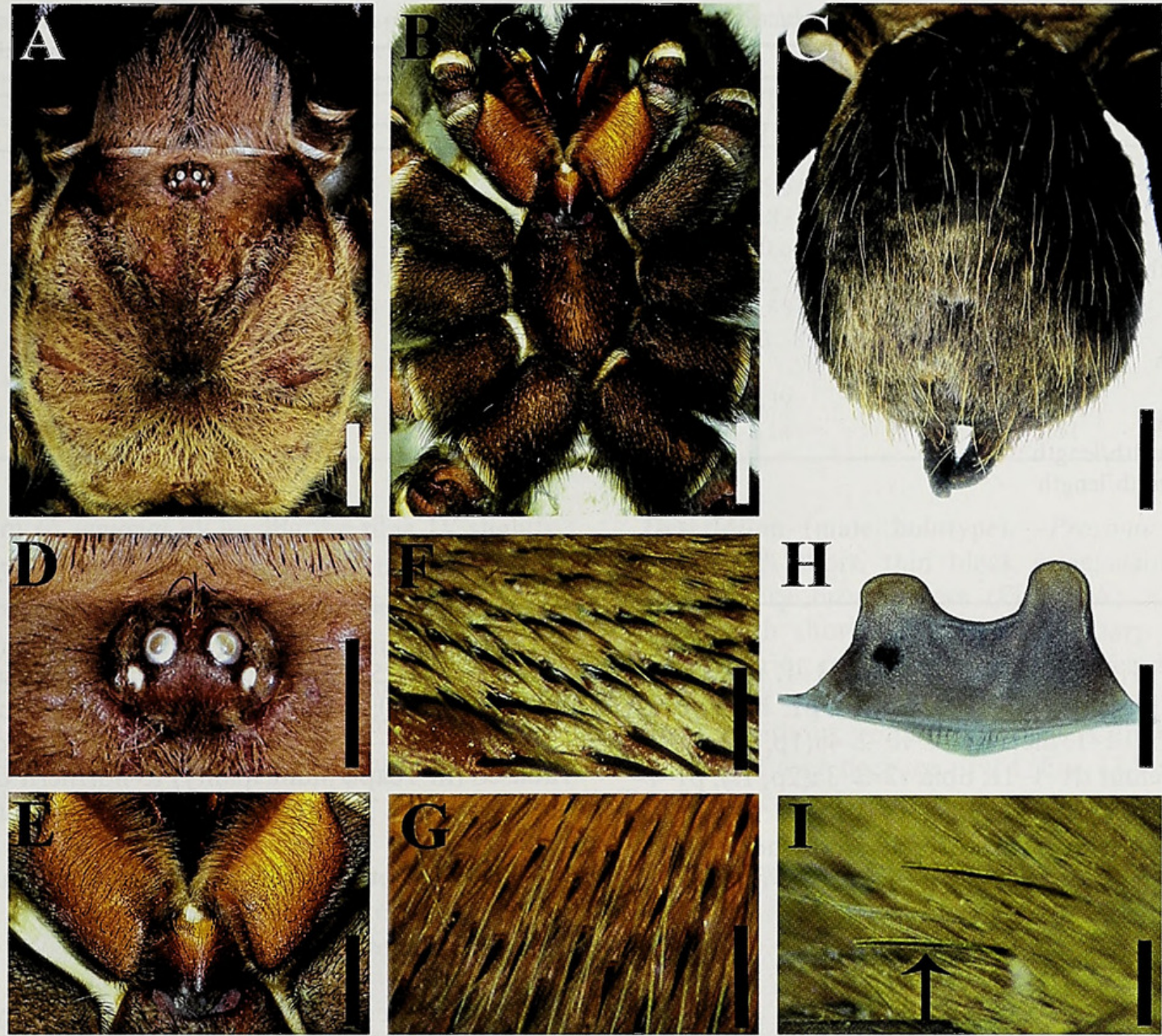

Figure 16.-Crassicrus cocona sp. nov. female paratype: A. Carapace. B. Prosoma, ventral view. C. Abdomen, dorsal view. D. Ocular tubercle E. Labium, maxillae, and labio-sternal mounds. F. Spiniform setae on prolateral face of coxa I. G. Spiniform setae on prolateral face of coxa IV. H. Spermathecae, dorsal view. I. Spiniform setae (arrow) on ventro-prolateral face of femur III. Scale bars: $0.5 \mathrm{~mm}(\mathrm{~F}-\mathrm{G}, \mathrm{I}), 2 \mathrm{~mm}(\mathrm{D}, \mathrm{H}), 4 \mathrm{~mm}$ (E), $5 \mathrm{~mm}(\mathrm{~A}-\mathrm{C})$

procurved; posterior eye row recurved (Fig. 16D). AME rounded, ALE and PME oval, PLE subtriangular. Ocular tubercle wider than long, with long setae on anterior and posterior regions (Fig. 16D); clypeus very narrow. Chelicerae longer than wide, surface covered with short coppery brown setae intermixed with long brown setae; dorsally with small iridescent setae and long white setae; dorso-prolateral region with very long setae that are basally brown and distally yellow. Prolateral furrow of chelicerae: left with 13 teeth (proximal to distal: 4, 11-13 largest; 1, 6, 9-10 medium-sized; 2, 3, 5, 7, 8 smallest); right with 16 teeth (proximal to distal: 4, 13-15 largest; 1, 7, 10-12, 16 medium-sized; 2, 3, 5, 6, 8, 9 smallest). Labium wider than long, with 36 cuspules anteriorly (Fig. 16E). Labio-sternal mounds as for holotype (Fig. 16E). Maxillae longer than wide; left with 234 cuspules, right with 250 cuspules on baso-prolateral region (Fig. 16E). Sternum as for holotype, surface covered with short grey setae and long black setae; sigilla not visible (Fig. 16B).

Legs: In live specimens, all segments are brown except for black femora. Ventral surface of coxae covered with short grey setae intermixed with short and long black setae. Coxae I-IV prolaterally covered with cuneiform thorn-like setae, slightly thicker ventrally, on coxae I-II larger ventrally (Fig. 16F), on coxae III-IV are weakly developed (Fig. 16G). Retrolateral superior surface of maxillae and coxae I-III sparsely covered with very short spiniform setae. Prolateral and prolaterodorsal surfaces of trochanters I-IV with elongated spiniform setae. Prolatero-ventral surface of femora I-IV with elongated spiniform setae (Fig. 16I). Patellae and tibiae with two bald, longitudinal stripes dorsally. Metatarsi I-III with a bald longitudinal stripe on dorsal face. All other segments are covered with short grey setae, and long setae that are basaily brown and distally yellow. Femur III slightly thickened with respect to femora I-II, IV. Tibia IV not thickened. Femur IV longer than metatarsus IV. Tarsal scopulae I-IV entire. Metatarsal scopulae I-III entire, IV divided by setae. Metatarsal scopulae extension: I complete; II 0.91; III 0.68 ; IV 0.24 .

Leg lateral scopulae: Pedipalp: $r$ (coxa, trochanter). Leg I: $p$ (coxa, trochanter, femur); r (coxa, trochanter). Leg II: p (coxa, trochanter, femur); r (coxa). Leg III: p (coxa, trochanter); r (coxa). Leg IV: p (coxa, trochanter); r (coxa, trochanter, femur).

Leg thin plumose setae: Pedipalp: $r$ (coxa, trochanter). Leg I: p (coxa, trochanter, femur); r (coxa, trochanter). Leg II: p (coxa, trochanter, femur).

Leg spination: Pedipalp: femur p0-0-1d, tibia v0 $0-4 \mathrm{a}(2 \mathrm{p}$, $2 \mathrm{r}$ ), p0-3-2. Leg I: tibia v0-0-1ap, p1-1-0, metatarsus v0-0- 
Table 9.--Variation in material examined for Crassicrus cocona sp. nov.

\begin{tabular}{|c|c|c|c|}
\hline \multicolumn{4}{|c|}{ Crassicrus cocona } \\
\hline Specimens & $\delta$ Holotype & 6 ㅇ Paratypes & 1 ơ Subadult \\
\hline Total length & 36.66 & $33.88-44.19$ & 39.79 \\
\hline Carapace length & 18.62 & $17.36-21.43$ & 18.76 \\
\hline Carapace width & 17.23 & $15.06-19.32$ & 16.14 \\
\hline Carapace width/length & 0.92 & $0.87-0.92$ & 0.86 \\
\hline Sternum length & 9.70 & $6.80-10.50$ & 8.8 \\
\hline Sternum width & 8.30 & $6.20-9.12$ & 7.75 \\
\hline Sternum width/length & 0.86 & $0.85-0.95$ & 0.88 \\
\hline Bulb length & 4.65 & - & - \\
\hline Embolus width/length & 0.52 & - & - \\
\hline Seminal receptacles width/length & - & $1.04-1.34$ & - \\
\hline Spermathecae base width/length & - & $2.07-3.10$ & - \\
\hline Chelicerae teeth & $14-15$ & $12-16$ & $13-16$ \\
\hline Labial cuspules & 53 & $36-71$ & 41 \\
\hline Maxillary cuspules & $162-175$ & $163-250$ & $131-140$ \\
\hline
\end{tabular}

1a. Leg II: patella p1, tibia v0 $0-3 a(1 \mathrm{p}), \mathrm{p} 1-1-1$, metatarsus v1-0-2a(1p). Leg III: femur d1-1-1r, patella p1, tibia v0-2$3 \mathrm{a}(1 \mathrm{p}, 1 \mathrm{r}), \mathrm{p} 1-1-1, \mathrm{r} 1-1-1$, metatarsus v0-2-4a(1p, 2r), p1-11, r0-1-1. Leg IV: femur d1-1-1r, tibia v2-2-3a(2p, 1r), p1-11, r1-1-1-1, metatarsus v22(5a), p1-1-1, r1-1-1.

Opisthosoma: Dorsal surface covered with short brown setae, interspersed with long yellow setae (Fig. 16C). Under the short brown setae, there is black pubescence, which corresponds to urticating setae.Ventrally covered with short and long black setae.

Urticating setae: Type I, with region "A" long and "B" short.

Genitalia: Spermathecae composed of two seminal receptacles partially fused by a heavily sclerotized median region with the median superior border slightly concave; each SB subquadrate, slightly wider than long (Fig. 16H); SS slightly wider than SB (Fig. 16H).

Measurements: Total length (prosoma + opisthosoma): 42.44. Leg span (measured from apex of left tarsus I to apex of left tarsus IV): 135.11. Carapace: length 21.43, width 19.32, carapace width/length 0.90 . Clypeus: 0.42 . Ocular tubercle: height 1.27, length 2.10, width 2.77. Eye sizes and interocular distances: AME 0.55; ALE $0.34 \times 0.52$; PME $0.27 \times 0.42$; PLE $0.29 \times 0.44$; AME-AME 0.28; AME-ALE 0.26; AME-PME 0.20, ALE-ALE 1.90, ALE-PME 0.34, PME-PME 1.49; PME-PLE 0.14; PLE-PLE 1.68; PLE-AME 0.55, PLE-ALE 0.38 . Fovea: width 3.37. Labium: length 3.25 , width 4.35 . Chelicerae: length 10.74 , width 8.56. Sternum: length 10.19 , width 9.12. Legs length (femur, patella, tibia, metatarsus, tarsus, total): I: $16.74,9.73,12.80,11.00,8.37,58.64$; II: 15.50, $8.87,10.65,10.71,7.85,53.58$; III: $14.30,8.13,9.86,11.72$, 7.47, 38.79, 51.38; IV: $18.57,8.67,12.86,16.95,8.49,65.54$. Pedipalp: $11.95,7.03,8.67,-, 9.56,37.21$. Leg formula IV, I, II, III. Leg widths: femora I-IV: 4.44, 4.39, 4.80, 4.43, pedipalp: 3.85 ; patellae I-IV: $3.99,3.93,3.84,3.93$, pedipalp: 3.40; tibiae I-IV: $3.20,3.14,3.26,3.49$, pedipalp: 3.08; metatarsi I-IV: $2.56,2.47,2.64,2.53$; tarsi I-IV: 2.11, 2.23, 2.37, 2.05, pedipalp: 2.57. Abdomen: length 21.01. Spermathecae: Base: length 2.04, width 4.25; SB: length 0.94, width 1.03; SS: width 1.40; SB-SB: 1.50. Spinnerets: PMS: length
2.50, width 1.25; PMS-PMS: 1.70; PLS: basal 4.45, median 2.65 , distal 4.30 ; width: $2.03,1.70,1.30$ respectively.

Distribution.- This species has been reported from southern Tabasco (in Teapa municipality) to northern Chiapas, near to the border with Tabasco (in Solosuchiapan municipality) (Fig. 2).

Natural history. - The specimens were found in straight burrows, approximately $30 \mathrm{~cm}$ deep. Two burrows were parallel and another two were perpendicular to the ground. The burrows had circular entrances and the vegetation surrounding them was covered with a thin layer of silk (Fig. 2B). The specimens were collected in April and the females did not have egg sacs. The holotype male was collected as an immature and its final molt in captivity was in early September. The locality where the specimens were collected was slightly disturbed, and it was between an area of very well conserved rainforest and cattle pastures.

Variation.- The number of visible sigilla is variable. In the holotype male, there are three pairs of sigilla, and, in female paratypes, there are from none to three pairs; if sigilla are visible, they are oval and small, with the third pair the largest. See Tables $7 \& 8$ for details of size variation in different characters. See Tables $9 \& 10$ for details of size variation in different characters.

Crassicrus yumkimil sp. nov. http://zoobank.org/?lsid=urn:lsid:zoobank. org:act:72B512D8-39C9-4468-B534-A9418244E761 (Figs. 17, 18)

Type material.-Holotype male. MEXICO: Campeche: $1 \mathrm{~km}$ West from El Pañuelo (Miguel de la Madrid), Candelaria municipality, $17.92422^{\circ} \mathrm{N}, 90.48160^{\circ} \mathrm{W}, 124 \mathrm{~m}, 16$ October 2011, O. Francke, A. Valdez, G. Montiel, D. Candia, D. Barrales (CNAN-T0938).

Etymology.- The specific name is a noun in apposition from the Mayan language. "Yum Kimil" is the Mayan god of death and it means "lord of the dead".

Diagnosis.-Crassicrus yumkimil sp. nov. can be distinguished from all other congeners except $C$. lamanai by having tibia IV thickened with respect to tibiae I-III. It is 
Table 10.- Variation in the lengths and widths of appendage segments for six adult females of the type series of Crassicrus cocona sp. nov. The segment with the data in bold was considered as thickened.

\begin{tabular}{|c|c|c|c|c|c|}
\hline Segment & Pedipalp & Leg I & Leg II & Leg III & Leg IV \\
\hline \multicolumn{6}{|l|}{ Length } \\
\hline Femur & $10.68-11.95$ & $15.05-16.74$ & $13.56-15.58$ & $12.54-14.37$ & $16.00-18.57$ \\
\hline Patella & $6.30-7.03$ & $8.63-9.73$ & $7.51-8.87$ & $7.11-8.13$ & $7.64-8.67$ \\
\hline Tibia & $7.85-8.67$ & $11.23-12.80$ & $9.83-10.65$ & $9.09-9.86$ & $12.10-12.86$ \\
\hline Metatarsus & - & $9.38-11.00$ & $9.03-10.71$ & $10.59-11.72$ & $15.43-16.95$ \\
\hline Tarsus & $8.53-9.56$ & $7.47-8.37$ & $7.11-7.85$ & $7.16-7.47$ & $8.14-8.49$ \\
\hline Total & $33.36-37.21$ & $51.76-58.64$ & $47.04-53.58$ & $46.72-51.48$ & $59.31-65.54$ \\
\hline \multicolumn{6}{|l|}{ Width } \\
\hline Femur & $3.23-3.85$ & $3.65-4.44$ & $3.65-4.39$ & $3.94-4.80$ & $3.70-4.43$ \\
\hline Tibia & $2.83-3.08$ & $3.01-3.20$ & $2.95-3.14$ & $3.02-3.51$ & $3.04-3.49$ \\
\hline
\end{tabular}

distinguished from C. lamanai by having the tibia IV slightly thickened, and by the presence of cuneiform, thorn-like setae on the retrolateral inferior faces of the maxillae and coxa I (Fig. 17F). The males can be further distinguished from $C$. lamanai by the presence of two keels (RM and RI) on the retrolateral face of the embolus (Fig. 18B), and by having striations on the prolateral face of the palpal bulb (Fig. 18A). Females are unknown.
Description (male holotype).-Prosoma: Dorsal surface covered with short, thin black setae; cephalic region with some coppery brown setae (Fig. 17A). Carapace margins covered with short grey setae and sharp spiniform brown setae. Posterior region of carapace covered with long, thick brown setae and long, soft white setae. Carapace semichordate, without pronounced boss. Caput slightly elevated (Fig. 17A). Fovea deep, recurved (Fig. 17A). Anterior eye row slightly procurved; posterior eye row recurved (Fig. 17D).
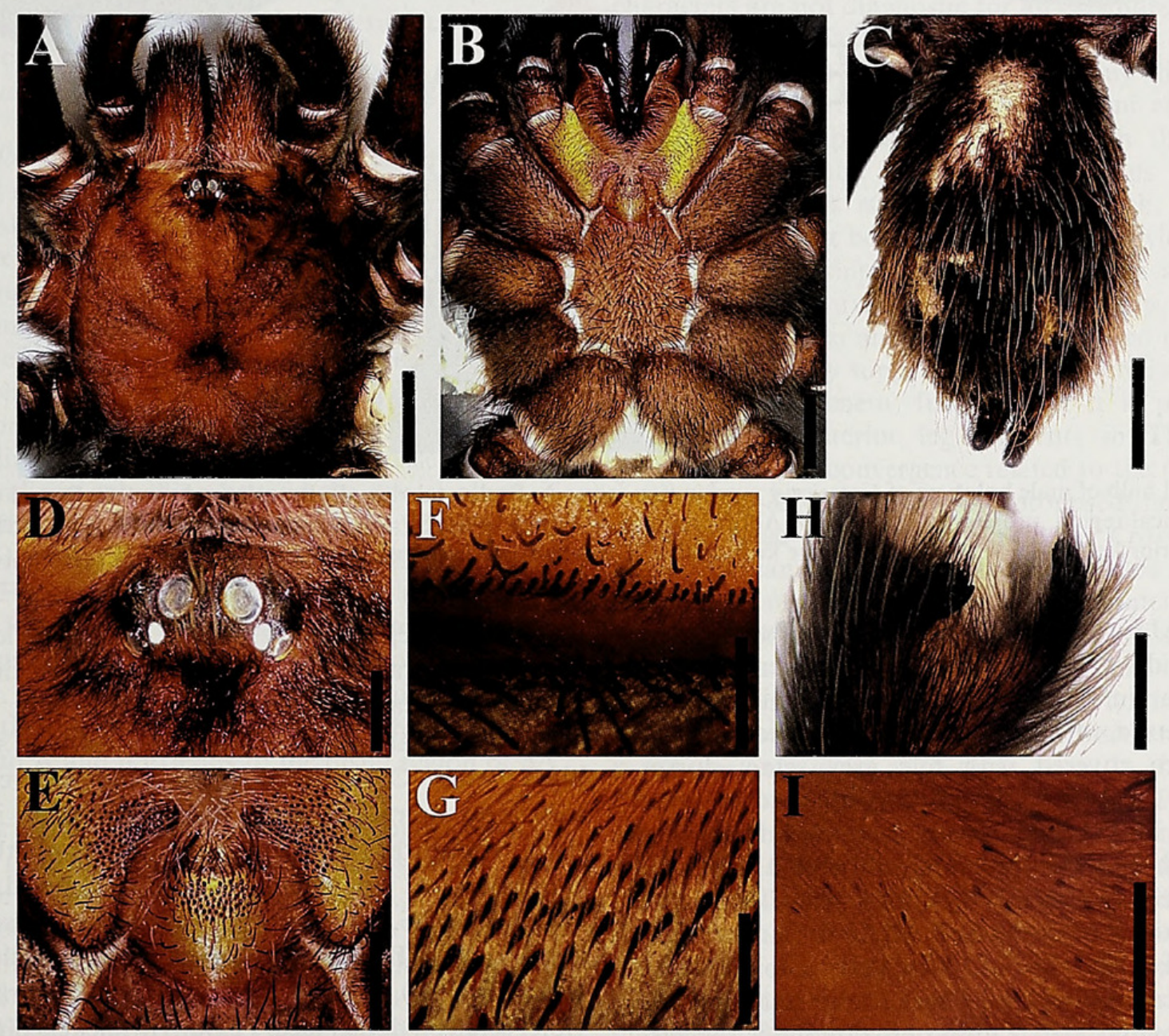

Figure 17.-Crassicrus yumkimil sp. nov. male holotype: A. Carapace. B. Prosoma, ventral view. C. Abdomen, dorsal view. D. Ocular tubercle. E. Labium, maxillae, and labio-sternal mounds. F, G. Spiniform setae on prolateral face of coxa I, (F) ventral view, (G) prolateral view. H. Tibia IV, dorsal view. I. Tibial apophysis, ventral view. J. Spiniform setae on retrolateral face of coxa I. Scale bars: $0.25(\mathrm{G}), 0.5 \mathrm{~mm}(\mathrm{~F}), 1$ $\mathrm{mm}(\mathrm{I}), 2 \mathrm{~mm}(\mathrm{D}, \mathrm{E}), 5 \mathrm{~mm}(\mathrm{~A}-\mathrm{C}, \mathrm{H})$. 


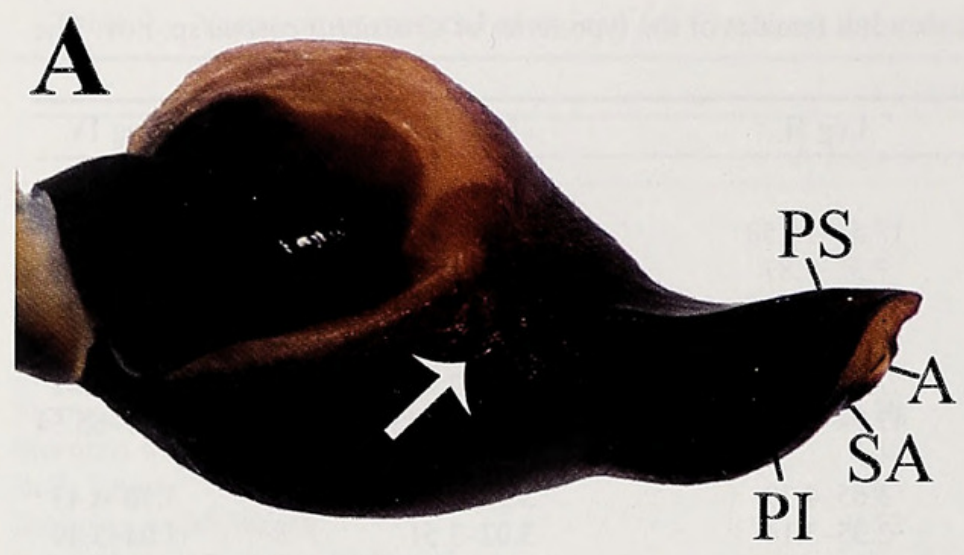

\section{B}

RM
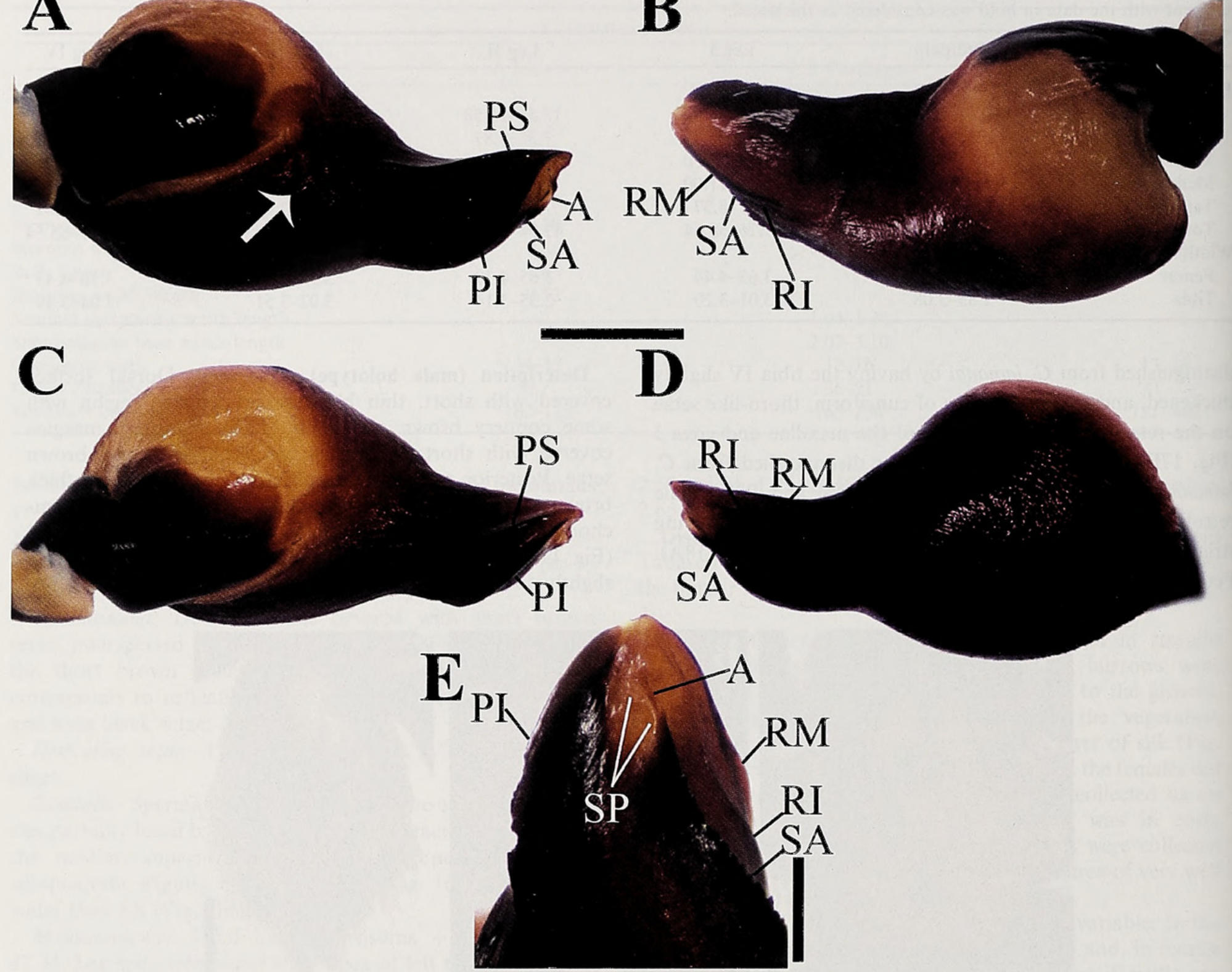

Figure 18.-Palpal bulb of male holotype of Crassicrus yumkimil sp. nov.: A. Prolateral view. B. Retrolateral view. C. Dorsal view. D. Ventral view. E. Embolus apical region, prolatero-ventral view. Abbreviations: $A=$ apical keel; $S P=$ spermatic pore keels; PI = prolateral inferior keel; $\mathrm{PS}=$ prolateral superior keel; $\mathrm{RI}=$ retrolateral inferior keel; $\mathrm{RM}=$ retrolateral median keel. Scale bars: $0.25 \mathrm{~mm}(\mathrm{E}), 1 \mathrm{~mm}(\mathrm{~A}-\mathrm{D})$.

AME rounded, ALE and PME oval, PLE subtriangular. Ocular tubercle wider than long; clypeus very narrow (Fig. 17D). Anterior margin of carapace covered with long, thin, black setae intermixed with thicker setae. Chelicerae longer than wide, surface covered with fine grey setae; dorsoprolateral region covered with fine coppery brown setae interspersed with long brown setae. Prolateral furrow of chelicerae: left with 13 teeth (proximal to distal: 1, 3, 11-13 largest; 6, 8-10 medium-sized; 2, 4, 5, and 7 smallest); right with 16 teeth (proximal to distal: 1, 3, 12, 14-16 largest; 4, 6, 9-11 medium-sized; 2, 5, 7, 8, and 13 smallest). Labium wider than long, surface covered with long dark brown setae; with 62 cuspules anteriorly (Fig. 17E). Labio-sternal mounds semicircular and separated (Fig. 17E). Maxillae longer than wide; left with 173 cuspules, right with 158 cuspules on baso-prolateral region (Fig. 17E). Sternum longer than wide, fiat (Fig. 17B); surface covered with fine grey setae intermixed with short and long dark setae; with three pairs of oval sigilla located close to baso-retrolateral face of coxae I, II, and III; third pair is the largest and located close to sternum edge (Fig. 17B).

Legs: Ventral surface of coxae covered with short and long black setae. Coxae I-IV prolaterally covered with short cuneiform thorn-like setae, thicker ventrally; in this species these spiniform setae are less abundant than in other congeners and are mainly basally distributed, near ventral edge (Fig. 17G, F). Retrolateral superior surface of maxillae and coxae I-III sparsely covered with very short spiniform setae (Fig. 17I). Coxae, trochanters, and femora covered with violet setae on dorsal face. Femur III thickened with respect to femora I-II, IV. Tibia IV slightly thickened with respect to tibiae I-III. Metatarsus IV longer than femur IV. Tarsal scopulae I-IV entire, IV divided with long setae. Metatarsal scopulae extension: I: complete, II: 0.90 , III: 0.60 , IV: 0.25 . 
Metatarsus I straight, when flexed touches the lateral face of Rap.

Leg lateral scopulae: Pedipalp: $r$ (coxa, trochanter). Leg I: $p$ (coxa, trochanter, femur); r (coxa). Leg II: p (coxa, trochanter, femur); r (coxa). Leg III: p (coxa); r (coxa). Leg IV: p (coxa); r (coxa, trochanter, femur).

Leg thin plumose setae: Pedipalp: $r$ (trochanter, femur). Leg I: p (trochanter, femur). Leg II: p (trochanter, femur).

Leg spination:Pedipalp: femur $\mathrm{d} 0-0-1 \mathrm{p}$, tibia v0-0-1, p2-73. Leg I: femur d0-0-1p, tibia v2-2-1a, p1-2-0, metatarsus v0-0-1a. Leg II: femur d0-0-1p, tibia v2-1-3a(1p), p1-1-1, metatarsus v1-0-2a, p1-0-0. Leg III: femur d0-0-2(1, 1r), p11-1, r0-1-1. Leg IV: femur d0-0-1r, tibia v1-4(1p)-3a(1p), p1-0-1-0, r1-1-1-1, metatarsus v14, p1-1-0-1, r0-1-1-2.

Leg I tibial apophyses: Tibia I with two branches that do not originate from a common base (Fig. 17H). Prolateral branch (Pap) short, longer than wide, retrolateral face with a megaspine that does not protrude apically (Fig. 17H). Retrolateral branch (Rap) almost two times longer than Pap, slightly curved towards it. Base subconical and distally digitiform; retroventral surface with a subapical megaspine that protrudes apically.

Opisthosoma: Dorsal surface covered with short thin dark brown setae intermixed with long setae (Fig. 17C). Under the short brown setae, there is coppery brown pubescence, which corresponds to the urticating setae. Ventral surface covered with short and long black setae.

Urticating setae: Type I, with region "A" long and "B" short.

Pedipalpal bulb: Bulb with striations on prolateral face of tegulum; ventral region with a shallow depression (Fig. 18A). Embolus short, slightly curved towards retrolateral face, with dorsal median region slightly concave and distally flat (Fig. 18A, C). Embolus with eight keels: (1) apical keel (A) very reduced and semitransparent (Fig. 18E); (2) subapical keel (SA) fully serrated, extending for more than half of embolus length and retrolaterally curved distally (Fig. 18B, D); $(3,4)$ prolateral inferior (PI) and prolateral superior (PS) keels sharp and wide, extending for more than half of embolus length (Fig. 18A); PS thin and not extending beyond the dorsal plane of embolus (Fig. 18A); $(5,6)$ retrolateral median (RM) and retrolateral inferior (RI) keels strong, slightly wider on their distal portion (Fig. 18B); extending for more than half of embolus length; RM distally fused with PS and together form the tip of embolus (Fig. 18B); (7, 8) Spermatic Pore keels (SP) semitransparent, surrounding the seminal duct opening, curved outside; the retrolateral is longer than the prolateral, curved, parallel to A and it extends to the distal region of SA (Fig. 18E).

Measurements: Total length (prosoma + opisthosoma): 28.43. Leg span (measured from apex of left tarsus I to apex of left tarsus IV): 127.48. Carapace: length 14.21, width 12.59 , carapace width/length 0.89 . Ocular tubercle: height 0.90 , length 1.50 , width 2.05 . Eye sizes and interocular distances: AME 0.42; ALE $0.35 \times 0.48$; PME $0.18 \times 0.26$; OLP $0.30 \times$ 0.32; AME-AME 0.26; AME-ALE 0.22; AME-PME 0.08, ALE-ALE 1.30, ALE-PME 0.42, PME-PME 0.96; PMEPLE 0.12; PLE-PLE 1.40, PLE-AME 0.36, PLE-ALE 0.32. Fovea: width 1.50. Labium: length 1.75 , width 2.55 . Chelicerae: length 7.16, width 4.54. Sternum: length 6.80 , width 6.00 .
Legs length (femur, patella, tibia, metatarsus, tarsus, total): I: $13.78,7.30,11.25,10.61,7.39,50.33$; II: $12.45,6.62,9.47,9.82$, 7.18, 45.54; III: $11.01,5.03,8.74,11.06,7.16,43.00$; IV: 13.70 , $5.91,11.48,15.87,8.64$. Pedipalp: $8.22,4.65,7.52,-, 3.17$, 23.56. Leg formula: IV, I, II, III. Leg widths: femora I-IV: $2.88,2.83,3.40,2.87$, pedipalp: 2.44 ; patellae I-IV: $2.62,2.63$, 2.71, 2.65, pedipalp: 2.20; tibiae I-IV: 2.09, 1.90, 2.17, 2.36, pedipalp: 2.55 ; metatarsi I-IV 1.91, 1.57, 1.54, 1.60; tarsi I-IV: $1.31,1.44,1.22,1.13$, pedipalp: 1.91. Abdomen: length 14.22 . Spinnerets: PMS: length 1.63 , width 0.67; PMS-PMS: 0.47; PLS: basal 2.57, median 1.55, distal 2.67; width: 1.05, 0.95, 0.67 respectively. Palpal bulb: length 3.65 ; tegulum: length 2.05 , height 1.85 ; embolus: length 1.60 , width 0.82 .

Distribution.-Known only from the type locality, in Campeche, México (Fig. 3).

Natural history. - The holotype male was collected in July, during the rainy season, when it was already aduit. It was found wandering on the surface near roadsides.

\section{DISCUSSION}

In the description of Crassicrus, Reichling \& West (1996) proposed diagnostic characters to distinguish the genus based only on the morphology of the type species $C$. lamanai. With the revision of new species, we observed that some of these characters are not diagnostic for an expanded Crassicrus. The thickening of tibia IV is only shared by $C$. lamanai and $C$. yumkimil, and, in C. yumkimil, it is only slightly thickened. According to Bertani (2001) and Bertani et al. (2011), this character state appears several times in the phylogeny of Theraphosinae, and the only genus thus far where it is uniformly present is Eupalaestrus Pocock, 1901. To date, Crassicrus has not been included in any phylogenetic analysis and the relationships between this genus and other Theraphosinae genera remain unclear. Similarly, the relationship between Crassicrus and Eupalaestrus, based on the incrassate tibia IV, is weakly supported since this character state occurs in some other genera; furthermore, it is possible that the widening of posterior leg segments in Theraphosidae in general is due to convergence related to the fossorial habitus.

Reichling \& West (1996) also proposed the presence of fine plumose setae as being diagnostic for Crassicrus. However, these setae can be found sparsely distributed or in patches on the retrolateral face of the palpal trochanter and femur, the prolateral surfaces of coxa I, trochanter I and femur I, the retrolateral surfaces of coxa $I$ and trochanter $I$, and on the prolateral surfaces of trochanter II and femur II, as indicated in the new species descriptions above. Furthermore, Crassicrus is not the only genus with these setae. In the description of Citharacanthus meermani Reichling \& West, 2000, the authors mentioned that $C$. livingstoni Schmidt \& Weinmann, 1996 and C. meermani have fine plumose setae on femora I and II (Reichling \& West 2000). By comparing SEM images of the fine plumose setae on the femur I of Cr. cocona (Fig. 1F) with those of the description of Ci. meermani, we found that the plumose setae have similar morphologies. In addition, material of the South American genera Lasiodora C. L. Koch, 1850 and Nhandu Lucas, 1983 were examined and also found to possess fine plumose setae on the lateral faces of the legs and pedipalps. According to Pérez-Miles et al. (2005), these setae can occur along with stridulatory setae as spines and 
claviform setae; however, because of the weak structure of the plumose setae, they seem to be unrelated to stridulation.

Other features that have been proposed as diagnostic for Crassicrus include the spiniform setae present on the prolateral surfaces of the leg coxae and on the ventral and proventral surfaces of femora II-IV (Reichling \& West 1996). These two kinds of setae are morphologically distinct. On the coxae, the spiniform setae are small, cuneiform, and are slightly larger and thicker close to the ventral region in both males and females. These spiniform coxal setae can also be found in species of other genera, such as Aphonopelma, Citharacanthus and Vitalius Lucas, Silva \& Bertani, 1993 (Hamilton et al. 2016; pers. obs.). However, these setae are usually thinner, longer and are not thick close to the ventral region as in other genera, or if they are, the spiniform setae are only present on coxae I-II. The second kind of spiniform setae are elongated and sharp, and are only present on the ventral and proventral surfaces of the leg femora of females. These two kinds of setae are present in virtually all Crassicrus species known (except the female of $C$. yumkimil). Therefore, we can confirm that these two characters are diagnostic for Crassicrus.

According to the revision of Theraphosinae palpal bulbs made by Bertani (2001), the male palpal bulb of $C$. lamanai has five keels: PS, PI, R, and probably two A keels, one of them dented. Our revision of the bulb of $C$. lamanai indicates that this species really has nine keels; and comparing it with the palpal bulbs of Eupalaestrus weijenberghi (Thorell, 1894), Nhandu coloratovillosus (Schmidt, 1998), Vitalius sorocabae (Mello-Leitão, 1923) and Lasiodora sp., we observed that the dented keel positioned on the ventral surface of the embolus of C. lamanai shares the same position and form as the SA keel found in the other species (both keels are positioned behind A keel, extend for more than half of the embolus length and both have small denticles). However, the homology of these two structures among the genera examined should be tested using a phylogenetic analysis.

In addition, we found that in Crassicrus, there are two or three retrolateral keels positioned on the inferior, median, and superior regions, respectively. Comparing the bulbs of Crassicrus with those of $E$. weijenberghi, $N$. coloratovillosus, $V$. sorocabae and Lasiodora sp., it is difficult to establish which of the three retrolateral keels found in the species of Crassicrus could be homologous to the single retrolateral keel found in the other genera. However, the revision of the bulbs of Citharacanthus meermani, which also has two retrolateral keels, indicates that the retrolateral inferior keel is very similar (morphologically and by position) to the keel present on the other genera. Currently, Crassicrus is the first genus where the presence of more than one retrolateral keel is constant; however, because this feature also appears in the species $C$. meermani, it cannot be considered diagnostic for the genus.

Ortiz \& Francke (2014) described for the first time the spermatic pore keels (SP) found on the ventral apex of the embolus of Bonnetina Vol, 2000, as two structures surrounding the spermatic pore. These two keels have not been reported in other genera; however, in Crassicrus, they are present in all species. These keels are shorter and heavily sclerotized, whereas in the two species of Bonnetina for which the keels have been described, they are straight and almost parallel. In Crassicrus, the prolateral SP keel is shorter, and the retro- lateral SP is curved, parallel to A keel, and extends to the apical region of the SA keel. The revision of specimens of other genera, such as Eupalaestrus and Lasiodora, indicates that these genera also have the SP keels, but that they are morphologically different from those observed in Crassicrus and Bonnetina; therefore, we recommend that these structures should be studied in further detail because they can provide potentially useful taxonomic information on the relationships among Theraphosinae.

Bertani and Guadanucci (2013) reported the different usage of the urticating setae types I and III in males and females, and the variation in the length of urticating setae across the abdominal area. Our examination of the urticating setae of males of Crassicrus revealed that in C. cocona, there are modified urticating type I setae on the median region of the abdomen of the male. According to Bertani and Guadanucci (2013), towards the median and posterior regions of abdomen, the length of the urticating setae increases. This elongation seems to be related to the more efficient use of these setae toward predator deterrence. In $C$. cocona the urticating type I setae on MM of the abdomen are more elongated than those of MA region, and have the region of the reversed barbs very reduced. The type III urticating setae seem to be more effective for defense against predators because they can be thrown easily; however, the type I urticating setae, due to the reversed barbs, get stuck to each other and cannot be thrown effectively. Therefore, the reduction of the reversed barbs in Crassicrus cocona could be an adaptation for the urticating setae to be thrown more efficiently (J. Guadanucci, pers. com.).

Considering the taxonomic revisions by Bertani $(2000,2001)$ and Bertani et al. (2011), we infer that the genus Crassicrus is probably phylogenetically related to the genera Lasiodora, Vitalius, Nhandu, Eupalaestrus, Proshapalopus Mello-Leitão, 1923, and Pterinopelma Pocock, 1901. These genera share the presence of R keel (in Crassicrus it is our RI keel), and share the slightly concave retrolateral face of the embolus above and below the retrolateral keel(s) (Bertani 2001). Additionally, Crassicrus females have spermathecae consisting of two receptacles partially fused by a heavily sclerotized median region, as do the females of Vitalius and Nhandu. However, we cannot test the placement of Crassicrus with these genera until performing a more thorough phylogenetic analysis.

\section{ACKNOWLEDGMENTS}

The first author thanks the Posgrado en Ciencias Biológicas of the Universidad Nacional Autónoma de México (UNAM) for support during the development of this project; the Consejo Nacional de Ciencia y Tecnología (CONACYT) for scholarship support during the master's degree project; and the Programa de Apoyo a los Estudios de Posgrado (PAEP) for financial support to facilitate a research visit to the American Museum of Natural History (AMNH). Finally, she thanks her tutorial committee for comments and advice given during this project: Atilano Contreras Ramos, and Javier Ponce Saavedra.

We are very grateful to Lorenzo Prendini for providing access to type and additional material deposited in the AMNH, and to Louis Sorkin and Lily Berniker for their help and hospitality during the research stay of the first author. We 
thank Jorge Mendoza and David Ortiz for their comments and suggestions that helped to improve the original version of the manuscript. We are grateful to Rick West both for providing the photographs of live specimens of Crassicrus lamanai and for his comments. And we thank the members of the Colección Nacional de Arácnidos (CNAN), and Colección Nacional de Ácaros (CNAC) of Instituto de Biología, UNAM for the support and help during field trips. We thank Jose Paulo Guadanucci for his comments about the modifications of urticating setae and their possible function. We thank Berenit Mendoza for her assistance with the SEM photographs. We also thank Fernando Pérez-Miles, Antonio Brescovit and Rogerio Bertani for the loan and/or donation of biological material for morphological revision. And finally, we are grateful to the Associate Editor and two anonymous reviewers for their constructive criticisms. Collections were made under Scientific Collector Permit FAUT-0175 from SEMARNAT to Oscar F. Francke.

\section{LITERATURE CITED}

Bertani, R. 2000. Male palpal bulbs and homologous features in Theraphosinae (Araneae, Theraphosidae). Journal of Arachnology 28:29-42

Bertani, R. 2001. Revision, cladistic analysis, and zoogeography of Vitalius, Nhandu, and Proshapalopus; with notes on other theraphosine genera (Araneae, Theraphosidae). Arquivos de Zoologia 36:265-356.

Bertani, R., R.H. Nagahama \& C.S. Fukushima. 2011. Revalidation of Pterinopelma Pocock, 1901 with description of a new species and the female of Pterinopelma vitiosum (Keyserling, 1891) (Araneae: Theraphosidae: Theraphosinae). Zootaxa 2814:1-18.

Bertani, R. \& J.P.L. Guadanucci. 2013. Morphology, evolution and usage of urticating setae by tarantulas (Araneae: Theraphosidae). Zoologia 30:403-418.

Bücherl, W. 1957. Sôbre a importância dos bulbos copuladores e das apófises tibiais dos machos na sistemática das aranhas caranguejeiras (Orthognatha). Anais da Academia Brasileira de Ciências 29:377-416.

Cooke, J.A.L, V.D. Roth \& E.H. Miller. 1972. The urticating hairs of theraphosid spiders. American Museum Novitates 2498:1-43.

Coyle, F.A. 1995. A revision of the funnelweb mygalomorph spider subfamily Ischnothelinae (Araneae, Dipluridae). Bulletin of the American Museum of Natural History 226:1-133.

Fukushima, C.S., R.H. Nagahama \& R. Bertani. 2008. The identity of Mygale brunnipes C.L. Koch, 1842 (Araneae, Theraphosidae), with a redescription of the species and the description of a new genus. Journal of Arachnology 36:402-410.

Gershman de P., B.S. \& R.D. Schiapelli. 1970. Discusión de los caracteres válidos en la sistemática de las aranas Theraphosomorphae. Bulletin du Muséum National d'Histoire Naturelle de Paris 41(Suppl. 1):150-154.

Goloboff, P.A. 1993. A reanalysis of mygalomorph spider families (Araneae). American Museum Novitates 3056:1-32.

Hamilton, C.A., D.R. Formanowicz \& J.E. Bond. 2011. Species delimitation and phylogeography of Aphonopelma hentzi (Araneae, Mygalomorphae, Theraphosidae): cryptic diversity in North American Tarantulas. PLoS ONE 6:e262707.

Hamilton, C.A., B.E. Hendrixon \& J.E. Bond. 2016. Taxonomic revision of the tarantula genus Aphonopelma Pocock, 1901 (Araneae, Mygalomorphae, Theraphosidae) within the United States. ZooKeys 560:1-340.

Locht, A. 2007. Estudio sobre la sistemática y distribución de la familia Theraphosidae (Arachnida, Araneae) en México. Facultad de Ciencias, Universidad Nacional Autónoma de México.
Mendoza-Marroquín, J.I. 2012. Bonnetina papalutlensis a new species of tarantula from Guerrero, Mexico, with notes on reproduction (Araneae, Theraphosidae, Theraphosinae). Revista Ibérica de Aracnología 20:57-62.

Mendoza-Marroquín, J.I. 2014. Taxonomic revision of Hemirrhagus Simon, 1903 (Araneae: Theraphosidae, Theraphosinae), with description of five new species from Mexico. Zoological Journal of the Linnean Society 170:634-689.

Ortiz, D. 2008. Description of Cubanana cristinae, a new genus and species of Theraphosinae tarantula (Araneae: Theraphosidae) from the island of Cuba. Boletín de la Sociedad Entomológica Aragonesa 42:107-122.

Ortiz, D. \& O.F. Francke. 2014. Two new species of Bonnetina tarantulas (Theraphosidae: Theraphosinae) from Mexico: contributions to morphological nomenclature and molecular characterization of types. Journal of Natural History 49:685-707.

Pérez-Miles, F., S.M. Lucas, P.I. da Silva Jr. \& R. Bertani. 1996. Systematic revision and cladistics analysis of Theraphosinae (Araneae: Theraphosidae). Mygalomorph 1:33-68.

Pérez-Miles, F., L. Montes de Oca, R. Postiglioni \& F.G. Costa. 2005. The stridulatory setae of Acanthoscurria suina (Araneae, Theraphosidae) and their possible role in sexual communication: an experimental approach. Inheringia, Série Zoologia 95:365-371.

Prentice, T. R. 1997. Theraphosidae of the Mojave Desert west and north of the Colorado River (Araneae, Mygalomorphae, Theraphosidae). Journal of Arachnology 25:137-176.

Raven, R.J. 1985. The spider infraorder Mygalomorphae (Araneae): cladistics and systematics. Bulletin of the American Museum of Natural History 182:1-180.

Raven, R.J. 1990. Comments on the proposed precedence of Aphonopelma Pocock, 1901 (Arachnida, Araneae) over Rechostica Simon, 1892. Bulletin of Zoological Nomenclature 47:126.

Reichling, S.B. 2003. Tarantulas of Belize. Krieger Publishing Company, Melbourne, Florida.

Reichling, S.B. \& R.C. West. 1996. A new genus and species of theraphosid spider from Belize (Araneae, Theraphosidae). Journal of Arachnology 24:254-261.

Reichling, S.B \& R.C. West. 2000. A new species of tarantula spider (Araneae, Mygalomorphae, Theraphosidae) from the Cayo District of Belize. Southwestern Naturalist 5:126-132.

Schiapelli R.D. \& B.S. Gerschman de P. 1962. Importancia de las espermatecas en la sistemática de las arañas del suborden Mygalomorphae (Araneae). Physis, Revista de la Sociedad Argentina de Ciencias Naturales (C) 23:69-75.

Schiapelli R.D. \& B.S. Gerschman de P. 1979. Las arañas de la subfamilia Theraphosinae (Araneae, Theraphosidae). Revista del Museo Argentino de Ciencias Naturales Bernardino Rivadavia (Ent.) 5:287-300.

Schmidt, G. 1997. Bestimmungsschlüssel für die Gattungen der Unterfamilie Theraphosinae (Araneae: Theraphosidae). Arachnologisches Magazin (Sonderausgabe) 3:1-27.

Schmidt, G. 2003. Die Volgespinnen. Westarp WissenschaftenVerlagsgellschacft mbH, Hohenwarsleben, Germany.

Schmidt, G. 2007a. Probleme mit der sicheren Bestimmung des Weibchens von Crassicrus lamanai Reichling \& West, 1996 (Araneae: Theraphosidae: Theraphosinae). Tarantulas of the World 125/126:8-9.

Schmidt, G. 2007b. Zur kenntnis der Theraphosinae-Weibchen mit verdicker Tibia IV. Spixiana 30:99-101.

Smith, A.M. 1995. Tarantula Spiders: Tarantulas of the U. S. A. and Mexico. Fitzgerald Publishing, London.

Valerio, C.E. 1980. Arañas terafósidas de Costa Rica (Araneae, Theraphosidae). I. Sericopelma y Brachypelma. Brenesia 18:259288.

Vol, F. 1999. A propos d'une spermatheque inhabituelle. Arachnides 42:1-13. 
World Spider Catalog. 2016. World Spider Catalog, Version 16.5. Natural History Museum, Bern. Online at http://wsc.nmbe.ch/ Yamamoto, F.U., S.M. Lucas \& A.D. Brescovit. 2012. Catanduba, a new Theraphosinae spider genus from Central Brazil (Araneae, Theraphosidae). Zootaxa 3172:1-19.

Manuscript received 13 January 2016, revised 7 October 2016.

\section{APPENDIX 1}

The following material was examined for comparative taxonomic purposes. Specimens are deposited in the CNAN, IBSP and MNHN.

\section{TAXONOMY}

Family Theraphosidae Thorell, 1870

Subfamily Theraphosinae Thorell, 1870

Eupalaestrus weijenberghi (Thorell, 1894)

Material examined.-URUGUAY: Dto. Canelones: 1 o, Salinas Norte, R39, Municipio Canelones, 2-3 March 2013, F.G. Costa
(FCE.MV 1190). Dto. Montevideo: 1 \&, Melilla, 9 March 2004, F.G. Costa, F. Pérez-Miles, Postiglioni (FCE·MV 1192).

\section{Lasiodora $\mathbf{s p .}$}

Material examined.-BRAZIL: Minas Gerais: 1 , Juiz de Fora, December, 1980, S. Lucas (IBSP 4588); 1 , same data except no collector (IBSP 3991). São Paulo: 1 ơ, Serra de Taubaté, J. L. Bagetto (IBSP 6375); 1 ๙, Taubaté, C. Bombeiros (IBSP 6395).

Nhandu coloratovillosus (Schmidth, 1998).

Material examined.-BRAZIL: Maranhão: $1 \delta$, Usina Hidroelétrica Estreito, Estreito municipality, 02 March 2011, M. Lima (CNANAr010129) ; 1 same data except 28 March 2011, J. Carneiro (CNAN-Ar010130).

Vitalius sorocabae (Mello-Leitão, 1923).

Material examined.-BRAZIL: São Paulo: 1 o, Iperó, M. A. Pepeira (CNAN-Ar010128). 1 $q$ without data (CNAN-Ar010127); 1 \&. Caucaia (CNAN-Ar010126).

Citharacanthus meermani Reichling \& West, 2000.

Material examined.-MEXICO: Quintana Roo: 1 o, Akumal, Tulum municipality, $20.39782^{\circ} \mathrm{N}, 87.31426^{\circ} \mathrm{W}, 4 \mathrm{~m}, 24$ November 2010, P. Bryant (CNAN-Ar004154). 


\section{$2 \mathrm{BHL}$ Biodiversity Heritage Library}

Candia-Ramírez, Daniela T and Francke, Oscar F. 2017. "Taxonomic revision of the genusCrassicrusReichling \& West, 1996 (Araneae: Theraphosidae:

Theraphosinae), with the description of additional keels on the embolus." The Journal of arachnology 45(1), 67-98. https://doi.org/10.1636/joa-s-16-005.1.

View This Item Online: https://www.biodiversitylibrary.org/item/274714

DOI: https://doi.org/10.1636/joa-s-16-005.1

Permalink: https://www.biodiversitylibrary.org/partpdf/289891

\section{Holding Institution}

Smithsonian Libraries

\section{Sponsored by}

Biodiversity Heritage Library

\section{Copyright \& Reuse}

Copyright Status: In Copyright. Digitized with the permission of the rights holder

Rights Holder: American Arachnological Society

License: https://creativecommons.org/licenses/by-nc-sa/4.0/

Rights: http://www.biodiversitylibrary.org/permissions/

This document was created from content at the Biodiversity Heritage Library, the world's largest open access digital library for biodiversity literature and archives. Visit BHL at https://www.biodiversitylibrary.org. 